\title{
A Hybrid Method for Inverse Obstacle Scattering Problems
}

\author{
Dissertation \\ zur Erlangung des Doktorgrades \\ der Mathematisch-Naturwissenschaftlichen Fakultäten \\ der Georg-August-Universität zu Göttingen
}

vorgelegt von

Pedro Miguel Picado de Carvalho Serranho

aus Lissabon, Portugal

Göttingen 2007 
D7

Referent:

Prof. Dr. Rainer Kress

Koreferent:

Prof. Dr. Roland Potthast

Tag der mündliche Prüfung: 


\begin{abstract}
We are interested in solving the time-harmonic inverse acoustic scattering problem. In this work, we present the state of the art of a new iterative method to numerically solve this problem. This iterative method is based on the idea of analytic continuation of the total field. The method can be applied to recover the position and shape of sound-soft or sound-hard obstacles and can be extended to the reconstruction of the position, the shape and the boundary impedance of the unknown obstacle from the knowledge of the scattered field at large distances (far-field pattern) for one single incident wave. The method under consideration combines ideas of both decomposition and iterative Newton methods and therefore is called a hybrid method. It does not need a forward solver and obtains good reconstructions from the far-field data for one single incident wave, achieving a good compromise between numerical accuracy, computational costs and required data. However, a good initial guess is needed to obtain numerical convergence. Moreover, within this thesis we discuss the convergence of the method by presenting two different theoretical approaches. The feasibility of the method and its robust behaviour for noisy data is exhibited by numerical examples.
\end{abstract}





\section{Zusammenfassung}

Wir sind an der Lösung des inversen Streuproblems für zeitharmonische akustische Wellen interessiert. In dieser Arbeit präsentieren wir den aktuellen Forschungsstand zu einem neuen iterativen Verfahren zur numerischen Lösung dieser Aufgabe, welches durch analytische Fortsetzung des Gesamtfelds begründet ist. Diese Methode kann die Position und Gestalt eines unbekannten Gebiets bei bekannter Randbedingung, sowie die Position, die Gestalt und die unbekannte Impedanzfunktion eines unbekannten Gebiets rekonstruieren. Als Daten benutzen wir dabei das Fernfeld einer einzigen einfallenden ebenen Welle. Dieses Verfahren kombiniert Ideen von Dekompositionsmethoden und von Newtoniterationen und wird daher als ein hybrides Verfahren bezeichnet. Es benötigt keinen direkten Löser und erzielt gute Rekonstruktionsergebnisse mit den Fernfelddaten für eine einzige einfallende Welle. Auf diesem Grund stellt dieses Verfahren einen guten Kompromiss dar zwischen numerischer Genauigkeit, Rechenkosten und Datenanforderung. Allerdings wird eine gute Ausgangsnäherung benötigt, um numerische Konvergenz zu sichern. Ferner untersuchen wir die Konvergenz des Verfahrens durch die Präsentation zweier verschiedener theoretischer Ansätze. Die Durchführbarkeit des Verfahrens mit exakten und fehlerbehafteten Daten wird durch numerische Beispiele belegt. 



\section{Acknowledgements}

I would like to state some words of acknowledgement to those who helped me making this work possible.

First of all I would like to thank Prof. Dr. Rainer Kress for his valuable advices and helpful discussions on the topic of this work, as well as for his availability to receive me and promptly answer my questions or clarify my doubts. I would also like to thank Prof. Dr. Roland Potthast for accepting the role of second referee.

The support of my work through a scholarship from the portuguese Foundation for Science and Technology (Fundação para a Ciência e Tecnologia) is gratefully acknowledge. I also acknowledge the support of the Graduiertenkolleg "Identification in mathematical models".

I would like to thank my office colleagues Harald Heese and Stefan Langer for the good work environment, for the exchange of ideas and knowledge in various topics and for helping me to adapt to the city of Göttingen. I also acknowledge the hospitality of the NAM institute and the University of Göttingen. My thanks are also extended to the small community of portuguese students in Göttingen, for sharing the feeling of my homeland.

My sincere thanks to my girlfriend Flávia Oliveira and to my good friends Carol and Oswaldo Ferreira for proofreading the use of english in parts of my thesis.

I also thank my girlfriend and my family for the support during the last three years. 



\section{Contents}

$\begin{array}{lll}1 & \text { Introduction } & 3\end{array}$

2 Acoustic Scattering Theory 13

2.1 The Helmholtz Equation . . . . . . . . . . . . . . . . . . . . 13

2.2 The Direct Acoustic Scattering Problem . . . . . . . . . . . . . . 15

2.2.1 Layer Potentials . . . . . . . . . . . . . . . . 21

2.2.2 Uniqueness and Existence theorems . . . . . . . . . . . . . 24

2.3 The Inverse Acoustic Scattering Problem . . . . . . . . . . . . . . . . 26

3 The Hybrid Method $\quad 33$

3.1 Previous Methods . . . . . . . . . . . . . . . . . . 33

3.2 The Hybrid Method's Basic Ideas . . . . . . . . . . . . . . . . . . 35

3.3 The Hybrid Method for the Dirichlet Case . . . . . . . . . . . . . . . . 37

3.4 The Hybrid Method for the Neumann Case . . . . . . . . . . . . . . . 42

3.5 The Hybrid Method for the Robin Case . . . . . . . . . . . . . . . . 56

4 Convergence Results $\quad 63$

$4.1 \quad$ A Related Minimization Problem . . . . . . . . . . . . . . . . . 63

4.2 A Related Newton's Method . . . . . . . . . . . . . . . . . . 67

5 Numerical Results $\quad \mathbf{7 7}$

5.1 Two-dimensional case . . . . . . . . . . . . . . . . . 78

5.1 .1 Direct problem . . . . . . . . . . . . . 78

5.1.2 Inverse Problem for the Dirichlet Case . . . . . . . . . . . . 85

5.1.3 Inverse Problem for the Neumann Case . . . . . . . . . . . . 90

5.1.4 Inverse Problem for the Robin Case . . . . . . . . . . . . . . . . 94

5.2 Three-dimensional case . . . . . . . . . . . . . . . . . . . . 98

5.2.1 Direct Problem . . . . . . . . . . . . . . . . . . . 99

5.2.2 Inverse Problem for the Dirichlet Case . . . . . . . . . . . . . . 104

6 Conclusions and Outlook 113 



\section{Chapter 1}

\section{Introduction}

The aim of this work is to present a hybrid method to numerically solve the inverse obstacle scattering problem. In this introductory chapter we will say some general words on inverse and scattering problems, as well as on methods to solve the particular inverse scattering problem under consideration. We will focus on the developments and state of the art of the hybrid method and briefly give an overview of the contents of the forthcoming chapters.

Two problems are called inverse to each other if the solution to the first is required to formulate the second and vice-versa. There are several simple examples of inverse problems, such as addition and subtraction or differentiation and integration. Usually one of them has been studied for a longer time and is therefore better understood than the other. In this way this problem has become easier to solve and is therefore called the direct problem. The inverse problem is then the remaining problem of the pair. Solving the inverse problem usually requires harder or even new techniques. In most cases the inverse problem is also ill-posed in the sense of Hadamard [17]. This means it fails to be uniquely solvable or that the solution does not depend continuously on the data. The failure of the latter property is a matter of major importance concerning developing numerical methods to solve the problem, since they have to somehow stabilize the illposedness of the problem. It is not seldom that mathematical problems arising from applications are ill-posed and therefore this is an area of great interest for several areas of science today.

The inverse problem that we consider in this paper - namely the inverse scattering problem - is in fact ill-posed. Scattering theory has been studied over the last century (see [2, 8, 9, 45]). In a general framework, Lax and Philips [45] described scattering theory as the comparison between the asymptotic behaviour of an evolving system as the time $t$ tends to $-\infty$ with its asymptotic behaviour as $t$ tends to $+\infty$. In particular, they were interested in considering systems constructed from a simple background system by the imposition of a perturbation (also called scatterer), provided that the influence of the perturbation would be negligible for large $|t|$. 
The numerical method we are going to present is to be applied to a subclass of these inverse problems. We are interested in considering obstacle scattering problems. Roughly speaking, the main concern of this problem is to study the effect that an obstacle (the scatterer) has on some incident wave. Moreover, one wants to study how this effect is influenced by the properties of the obstacle, namely its shape, location and physical constitution. We consider an obstacle $D$ (the scatterer) embedded in some known homogeneous background. Knowing the physical properties of the background medium and

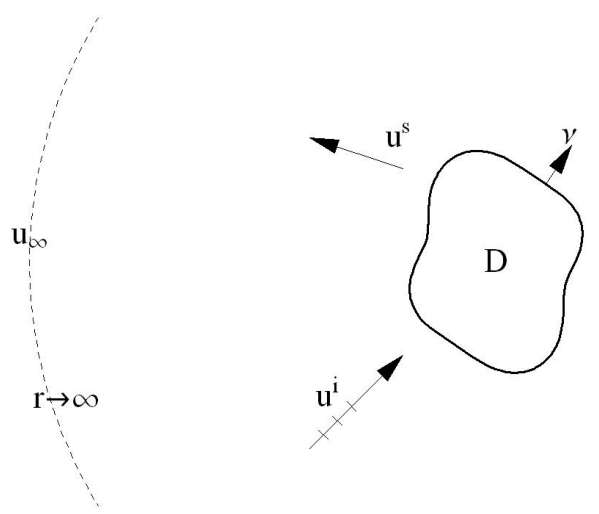

the obstacle, the direct problem is to determine the scattered wave $u^{s}$ given an incident wave $u^{i}$. The inverse problem we are interested in is to recover some physical properties of the obstacle $D$, such as its shape, location or physical constitution, given the measured scattered field at large distances. This inverse problem has been studied closely over the past 25 years (e.g. $[8,9,24,28,58])$. The mathematical model for this inverse problem is motivated from several areas of physics and engineering and is mainly related to non-destructive testing. Among the many applications we mention radar, sonar, mine detection or medical imaging.

We will shortly introduce the mathematical foundations of these problems (for details see chapter 2 and the references therein). We are interested in the special case of timeharmonic acoustic scattering as motivated in section 2.1, that is, we are interested only in the space dependence $u^{s}$ of the scattered wave. From now on we consider $D \subset \mathbb{R}^{m}$, for $m=2,3$, to be an open bounded obstacle with a $C^{2}$-smooth boundary and an unbounded and connected complement. Then, given an incident field $u^{i}$, the direct scattering problem consists of finding the total field $u=u^{i}+u^{s}$ as the sum of the known incident field $u^{i}$ and the scattered field $u^{s}$ such that both the Helmholtz equation

$$
\Delta u+k^{2} u=0 \quad \text { in } \mathbb{R}^{m} \backslash \bar{D}
$$

with wave number $k>0$ and the boundary condition

$$
B u=0 \quad \text { on } \Gamma:=\partial D
$$


are satisfied. The operator $B$ defines the boundary condition that is to be satisfied and is related with the physical properties of the obstacle $D$. For $B u=u$ we have a Dirichlet boundary condition while for $B u=\partial u / \partial \nu$ we have a Neumann boundary condition, where $\nu$ stands for the exterior normal vector to $\Gamma$. These two boundary conditions model the case of sound-soft and sound-hard obstacles, respectively. Usually obstacles are not perfectly sound-soft nor perfectly sound-hard, so it makes sense to impose an impedance or Robin boundary condition corresponding to $B u=\partial u / \partial \nu+i \lambda u$ with some impedance function $\lambda>0$.

Since this is an exterior problem, to ensure uniqueness of the solution (and therefore well-posedness in the sense of Hadamard) a condition at infinity needs to be imposed. Sommerfeld [63] suggested the radiation condition

$$
\lim _{r \rightarrow \infty} r^{\frac{m-1}{2}}\left(\frac{\partial u^{s}}{\partial r}-i k u^{s}\right)=0, \quad r=|x|
$$

with the limit satisfied uniformly in all directions. The physical meaning of this condition is that there are no energy sources at infinity. With this radiation condition the direct problem is well-posed (e.g [9]), since it is uniquely solvable and the scattered wave depends continuously on the incident field. It can also be shown that the solution $u^{s}$ to the direct problem can be represented by Green's representation formula

$$
u^{s}(x)=\int_{\Gamma}\left(u^{s}(y) \frac{\partial \Phi(x, y)}{\partial \nu(y)}-\frac{\partial u^{s}}{\partial \nu}(y) \Phi(x, y)\right) d s(y), \quad x \in \mathbb{R}^{m} \backslash \bar{D} .
$$

under some assumptions (see thm.2.3), where $\Phi$ stands for the fundamental solution to the Helmholtz equation. Moreover, one can show (see thm. 2.4) that the solution $u^{s}$ has an asymptotic behaviour of the form

$$
u^{s}(x)=\frac{e^{i k|x|}}{|x|^{\frac{m-1}{2}}}\left(u_{\infty}\left(\frac{x}{|x|}\right)+O\left(\frac{1}{|x|}\right)\right), \quad|x| \rightarrow \infty,
$$

where the function $u_{\infty}$ defined on the unit sphere $\Omega$ is called the far-field pattern and uniquely determines the scattered field $u^{s}$.

The inverse problem we are interested in is to reconstruct some properties of the obstacle from the knowledge of the scattered field at large distances generated by a known incident field. In this sense we will assume the far-field pattern as the given data. With the a priori knowledge that the obstacle is sound-soft or that it is sound-hard, the inverse problem can then be formulated as follows: Given an incident field $u^{i}$ and the corresponding far-field pattern $u_{\infty}$, determine the position and shape of the obstacle $D$. Without this a priori information on the boundary condition, the problem can be formulated with an impedance boundary condition where the impedance $\lambda$ is unknown. Note that the Neumann boundary condition is a special case of the impedance condition for $\lambda=0$, as well 
as the Dirichlet boundary condition can be seen as the limit of an impedance condition as $\lambda \rightarrow \infty$. Therefore, in this case where the boundary condition is unknown, the inverse problem is formulated in the following way: Given an incident field $u^{i}$ and the corresponding far-field pattern $u_{\infty}$, determine the position and shape of the obstacle $D$ and the impedance function $\lambda$ over its boundary. Uniqueness results for this inverse problem are still open in a general setting considering data for one single incident wave. However, some results can be obtained for sound-soft obstacles with size constraints (see thm.2.18) or special shapes (see $[1,6]$ ). Moreover, this inverse problem is non-linear in the sense that the scattered field depends non-linearly on the obstacle and it is also ill-posed in the sense that the determination of $D$ does not depend continuously on the far-field pattern. In this way, each numerical method considered to solve the inverse problem must take these two difficulties into account.

As already mentioned, there are several different applications for this kind of problems and therefore several methods were suggested to deal with the specific properties of each problem. For instance, for mine detection one can only measure the scattered wave on one side of the obstacle and usually has no a priori information on where the obstacle lies. On the contrary, if one wants to test whether some object is broken inside, one can usually measure the data all around the obstacle, and one also has an idea of how the obstacle should look to start iterating from it.

In the literature, one usually classifies the methods for inverse scattering within three classes: iterative methods, decomposition methods and sampling or probe methods. In this introductory chapter we will only say some words on the first two classes and we refer to section 3.1 and the references therein for more details.

Iterative methods usually work on a Newton method's idea, in the sense that from an ill-posed operator equation equivalent to the inverse problem they arrive at a linearized and still ill-posed equation which is solved using some regularization scheme. With $u_{\infty}$ the measured far-field pattern generated by the incident field $u^{i}$, this can be done considering the equation

$$
F(\Gamma)-u_{\infty}=0
$$

where the operator $F$ maps the boundary $\Gamma$ of some obstacle $D$ to the far-field pattern corresponding to scattering by $D$ with the same fixed incident field $u^{i}$. Then, due to the Fréchet differentiability of $F$, the above equation is solved by regularized Newton iterations. In case of convergence, it is clear that this method will only converge to a local minimum, so it is crucial to have a good initial guess. Moreover, as the Fréchet derivative of $F$ is characterized in terms of the solution to a forward problem, this method requires some extra computational effort due to the required forward solver. The characterization of $F^{\prime}$ depends also on the boundary condition, so the a priori knowledge of the boundary condition is required. Nonetheless in [40] this method was generalized for the case of an unknown impedance $\lambda$. The strong point about this method is that the reconstructions obtained are usually very good. As for convergence results, though some work has been 
done in that direction $[20,21,22]$, it is still considered to be an open problem in the sense that some of the assumptions on the operators are not yet proven for the case of inverse scattering.

As for decomposition methods, they split the ill-posedness and non-linearity of the problem in two steps. In an ill-posed first step the scattered field is reconstructed from the measured far-field data. In the Kirsch and Kress decomposition method [30, 31, 32] this is done by representing the scattered field $u^{s}$ as an appropriate layer potential over some appropriate $a$ priori chosen surface $\gamma$. The density for the layer potential is such that the far-field pattern of the layer representation fits the given measured far-field pattern. Then, in a non-linear second step, the position and shape of the scatterer is found by the location of the zero level set of the boundary condition. This is done by linearizing the $L^{2}$-norm of the boundary condition and finding a surface as a minimal solution to this linearized equation in a least squares sense. It is then clear that this method can only be applied in the case where the boundary condition is known a priori. The main advantage of this method is that no forward solver is needed. However, the numerical reconstructions are not as good as for iterative methods. Moreover, there is also a gap between numerical implementation and theoretical background. One can prove convergence for a related minimization problem, which is given in terms of a functional that is the sum of the minimization of the first and second step of the method. In this way, it is not clear that the minimum obtained by minimizing the first and second step independently or by simultaneous minimization is the same.

In the meantime two methods appeared that combine ideas of both these classes. On the one hand, although they are of iterative nature, they do not need a forward solver. On the other hand, they can compete with Newton's iterative methods concerning the numerical reconstructions obtained. The first method was suggested by Kress in [38]. As being the descendent of two different classes of methods, this method was called hybrid and will be the main topic of this thesis. The hybrid method is closer to a decomposition method and can be seen as an iterative and more competitive version of the Kirsch and Kress method [30, 31, 32]. The second method was suggested by Kress and Rundell [41] and developed for inverse scattering problems by Ivanyshyn and Kress [25]. This method is closer to an iterative Newton method, with the linearization made on the layer potential over the boundary instead of in the far-field operator $F$. In this way a forward solver is no longer needed, since no characterization of $F^{\prime}$ is now required.

\section{The Hybrid Method}

The main topic of this thesis is the development of the hybrid method in recent years, both from a theoretical and a numerical point of view.

The basic ideas of the method are the following (for details see section 3.2). Being $\gamma_{n}$ the current approximation to the boundary $\Gamma$, in a first step one starts by approximating the scattered field as a layer potential over $\gamma_{n}$, as in the Kirsch and Kress method men- 
tioned previously. The density of the layer representation is chosen so that the far-field pattern of the layer representation fits the given measured far-field pattern. In this way, the density is given as a solution to an ill-posed integral equation. In a second step, having in mind the fact that the boundary condition is to be satisfied over $\Gamma$, one defines the operator

$$
G:\left.\gamma \mapsto B u\right|_{\gamma}
$$

that maps a curve $\gamma$ onto the trace of the given boundary condition over $\gamma$. Note that the correct boundary $\Gamma$ is the solution to $G(\Gamma)=0$. In this way, making use of the Fréchet differentiability of $G$, one linearizes the previous equation around the current approximation $\gamma_{n}$. One now solves the linearized equation in a least squares sense in order to obtain a new approximation $\gamma_{n+1}$, using as approximation to the total field the layer representation obtained in the first step. One can now iterate both steps until some stopping criterion is achieved.

It is clear that this method combines ideas of both iterative and decomposition methods. On the one hand, it splits the ill-posedness from the non-linearity of the problem. On the other hand, it makes use of the Fréchet differentiability of the operator $G$ in order to linearize a non-linear equation and allow an iterative procedure, in the spirit of an iterative Newton method. Moreover, it gains advantages from both of them. No forward solver is needed and the quality of the reconstructions is quite good. Unfortunately, some disadvantages are also inherited in the hybrid method. A good initial guess is needed and, at first sight, it seems that the boundary condition must be also known a priori. However, this last disadvantage can be mended as we will mention in a few lines (and later on in detail in section 3.5).

To implement this new approach, the characterization of the Fréchet derivative of $G$ had to be given. Moreover, this characterization depends on the boundary condition. The proofs for its characterization are very simple for the Dirichlet case (see thm. 3.6) but become rather technical and involved for the Neumann case (see thm. 3.11 and cor. 3.12 for $\mathbb{R}^{2}$ and thm. 3.13 for $\mathbb{R}^{3}$ ). For the Robin boundary condition one just needs to combine both previous characterizations. However, the main tool used is Taylor's expansion, since one assumes the total field $u$, as a solution to the Helmholtz equation, to be analytic in a neighbourhood of $\gamma_{n}$.

The hybrid method can also be seen as an iterative version of the Kirsch and Kress decomposition method $[30,31,32]$. In some sense, the hybrid method is an attempt to revive the Kirsch and Kress decomposition method in a more competitive version. In fact, while in the latter the surface $\gamma$ has the role of an auxiliary surface used only to reconstruct the total field, in the hybrid method we get iteratively a sequence of surfaces $\gamma_{n}$ that are the current approximations to the correct boundary $\Gamma$. The hybrid method is therefore more effective, as shown in the numerical examples in section 5. Moreover, this change allows new features to the method. While in the Kirsch and Kress method the auxiliary surface $\gamma$ was required to be inside the scatterer $D$ in order to have an approximation to 
the total field valid in a neighbourhood of the correct boundary $\Gamma$, in the hybrid method the initial guess is allowed to be inside, outside or even intersecting the boundary $\Gamma$, since at each step the field $u$, along with its derivatives, is only evaluated on the current approximation $\gamma_{n}$. In addition, this change also allows the method to be applied to the case of a Robin boundary condition with unknown boundary impedance. This is done by considering the operator

$$
G:\left.(\gamma, \zeta) \mapsto\left(\frac{\partial u}{\partial \nu}+i \zeta u\right)\right|_{\gamma}
$$

that maps a curve $\gamma$ and an impedance function $\zeta$ to the corresponding impedance boundary condition. Again we want to find a pair $(\Gamma, \lambda)$ such that $G(\Gamma, \lambda)=0$. In this way, with $\left(\gamma_{n}, \zeta_{n}\right)$ being our current approximation, we linearize the previous equation both in the boundary and impedance variables around $\left(\gamma_{n}, \zeta_{n}\right)$, and using an approximation to the total field obtained in a first step (just as in the a priori known boundary condition case), we obtain a new approximation $\left(\gamma_{n+1}, \zeta_{n+1}\right)$ to the correct pair $(\Gamma, \lambda)$. In this way the hybrid method also represents a valid alternative to the Newton iterative method [40] as another iterative method that recovers both the boundary $\Gamma$ and the impedance $\lambda$ simultaneously.

The appearance and development of a new method must also include some theoretical background, namely in what concerns its convergence. However, as in general for numerical methods applied to the inverse obstacle scattering problem, the convergence results for the hybrid method are not completely satisfying. We consider and present in chapter 4 two main approaches. The first one is relating the hybrid method to a minimization problem (see section 4.1), as considered by Kirsch and Kress for their decomposition method. The advances made in the theory with this approach included generalizing the results obtained by Kirsch and Kress (see [9, sec.5.4]) for a combined layer representation and for the cases of the Neumann and Robin boundary condition. As a result, one can prove that the minimization problem has a unique solution for every given data and that if the given data is exact then the solution to the minimization problem satisfies the boundary condition in the sense that, given a sequence of regularization parameters decreasing to zero, there exists a subsequence of the corresponding solutions to the minimization problem that converges to a limit where the boundary condition is satisfied. However, as for the Kirsch and Kress method, there is a gap between this minimization problem and the numerical implementation of the hybrid method. In fact, as already referred, it is not clear that the minimization in two steps of the hybrid method (first on the density of the layer potential and secondly on the surface $\gamma$ and possibly on the impedance $\zeta$ if one considers the Robin boundary condition) is equivalent to a minimization in one single step (both on the density of the layer potential and the surface $\gamma$ and possibly also on the impedance $\zeta$ ) considered in the related minimization problem. Moreover, the minimization problem does not take into account the iterative procedure of repeating the two steps nor the linearization in the second step. In this way a new approach was needed. There- 
fore, in a second approach presented in section 4.2 the hybrid method is interpreted as a Newton method. The idea of proof is based on [55], but the operators appearing are quite different. This approach takes into account the linearization in the second step, as well as the iterative procedure, being a quite fair representation of the numerical implementation of the hybrid method. Under some assumptions, one can prove convergence for exact data as well as convergence to the correct solution with noisy data as the noise level goes to zero. However, this result can only be applied in some cases, since the assumptions include higher smoothness of $\Gamma$ and that the radial derivative of the total field $u$ does not have zeros on $\Gamma$. One can show numerically that this last assumption holds for domains close to circles (see remark 4.9). Moreover, this approach can only be applied to sound-soft obstacles, with no obvious possibility of extension to the Neumann and Robin boundary conditions.

The results on the hybrid method have also been published in journals while they were being achieved. As already mentioned, the basic idea of the hybrid method was suggested in [38], as a combination of ideas of both iterative and decomposition methods in order to create an iterative method using as background idea analytic continuation of the total field. The same idea was applied to an inverse boundary value problem in potential theory [5]. In [42] the hybrid method was generalized to the case of inverse scattering for sound-soft cracks. An important aspect about the latter paper is that it eliminated a gap in the traditional Kirsch and Kress method concerning crack reconstruction, since in the second step of the method the approximation surface could degenerate to a point, minimizing in this degenerated way the $L^{2}$-norm of the total field over the approximation surface. The hybrid method was then generalized for sound-hard obstacles [43], with the Fréchet derivative of $G$ being then characterized for the Neumann boundary condition. Later this method was also extended to scattering for shape and impedance [59], recovering both the obstacle and the unknown impedance on its boundary. All the previously mentioned papers concerning the hybrid method presented numerical results in $\mathbb{R}^{2}$. The numerical implementation in $\mathbb{R}^{3}$ for the Dirichlet case will appear shortly [60].

\section{Organization of the Thesis}

This thesis is organized in six chapters. In the second chapter the basic results of acoustic scattering theory are presented. We start by motivating the Helmholtz equation in section 2.1. We then proceed by presenting the direct acoustic scattering problem, recalling some fundamental results including Green's representation formula, layer potentials and their properties and uniqueness and existence results for the direct problem. In section 2.3 the inverse obstacle scattering problem is formulated and the state of the art concerning uniqueness results is presented.

Chapter 3 is dedicated to the ideas of the hybrid method. After section 3.1, where previous numerical methods for the inverse scattering problem are discussed, we present in section 3.2 the basic ideas of the hybrid method. We stress assumption 3.2 of the ana- 
lytic continuation principle and the advantages and disadvantages of this new method in comparison with previous methods. In the following sections 3.3-3.5 a detailed characterization of the hybrid method is given for the Dirichlet, Neumann and Robin boundary conditions, respectively. This includes the characterization for each considered boundary condition of the Fréchet derivative of the operator $G$ mentioned previously, these being the fundamental results for the numerical implementation of the hybrid method. Note also that at the end of section 3.5, after the characterization of $G^{\prime}$ has been established, the characterization of $G^{\prime}$ is compared with the characterization of the Fréchet derivative of the boundary-to-far-field operator $F$. Resemblances between $F^{\prime}$ and $G^{\prime}$ are found when evaluating $G^{\prime}$ over the correct boundary $\Gamma$ (and impedance $\lambda$ ) and are justified in that section.

Convergence results for the hybrid method are the topic of chapter 4. In section 4.1, the hybrid method is related with a minimization problem, for which convergence is shown. In the following section, namely section 4.2, the hybrid method is interpreted as a Newton method and convergence is proven under some assumptions. This latter approach is a better representation of the numerical implementation of the hybrid method, but it only works for sound-soft obstacles.

The feasibility of the method is then shown is chapter 5 through numerical examples. We divide the approach for $\mathbb{R}^{2}$ and $\mathbb{R}^{3}$, presenting in both cases how the synthetic farfield data was generated by solving the direct problem. This will also serve as the basis for the numerical quadrature rules used to implement the hybrid method. The numerical implementation of the hybrid method is then presented in $\mathbb{R}^{2}$ for each of the considered boundary conditions in sections 5.1.2-5.1.4. The numerical results with exact and noisy data are presented at the end of each section. The hybrid method for sound-soft obstacles in $\mathbb{R}^{3}$ is treated in section 5.2.2.

In the final chapter we make some considerations and final conclusions on the hybrid method and present some future perspectives. 



\section{Chapter 2}

\section{Acoustic Scattering Theory}

Scattering theory has been a matter of interest for scientists over the last century. There is a broad band of applications, such as radar and sonar or medical imaging. Roughly speaking, scattering theory studies the effect that an obstacle or some inhomogeneity has on an incident wave or particle. Considering the total field $u$ to be the sum of the incident field $u^{i}$ and the scattered field $u^{s}$, then the direct problem consists of determining $u^{s}$ from the knowledge of the medium and the propagation of the field, or stated in mathematical terms from the knowledge of the obstacle or inhomogeneity, including the boundary condition satisfied at the boundary of the obstacle, and the differential equation that rules the propagation of the field. The inverse problem is however a much more challenging and interesting problem: Given information on the scattered field $u^{s}$ one wants to find some unknown properties of the obstacle, such as its location and shape, the boundary condition or some refractive index, for instance. We refer to the monographs Lax and Philips [45] and Colton and Kress [8,9] for further reading on the basic theory of some of these problems.

In this work we do not intend to cover all these problems but to confine ourselves to the acoustic time-harmonic obstacle scattering problem within an homogeneous background. In order to do so we will motivate the Helmholtz equation as the model to this problem in the next section. Then in the following sections we will present classical theoretical results for the direct and inverse scattering problems, including representation formulas and asymptotic behaviours for the scattered field $u^{s}$ and uniqueness and existence results for the referred problems.

\subsection{The Helmholtz Equation}

We start by giving a motivation to the Helmholtz equation

$$
\Delta u+k^{2} u=0
$$


for $k>0$ as being a model to the space dependence of the limit state of a time harmonic acoustic wave with a point source excitement. We refer to [16] or the classical work of Lax and Philips [45] for details.

Consider the wave equation with a point source at $y$. This means that the system is at rest and at $t=0$ the harmonic excitement is started at the point $y$ in free space. In mathematical terms this can be formulated in terms of an acoustic wave $U^{i}(x, t ; y)$, depending on the space variable $x \in \mathbb{R}^{3}$ and the time variable $t \geq 0$, satisfying the equation

$$
\frac{1}{c^{2}} \frac{\partial^{2} U(x, t)}{\partial t^{2}}-\Delta_{x} U(x, t)=e^{-i \omega t} \delta(x-y), \quad x \in \mathbb{R}^{3}, t \geq 0
$$

with initial conditions

$$
U(x, 0)=\frac{\partial U(x, 0)}{\partial t}=0,
$$

where $c$ is the speed of sound and $\omega$ is the frequency. We are interested in studying the behaviour of the solution as $t \rightarrow \infty$. Then one can show the asymptotic behaviour

$$
U^{i}(x, t) \approx e^{-i \omega t} \Phi(x-y), \quad t \rightarrow \infty
$$

where $\Phi$ is the fundamental solution to the Helmholtz equation in $\mathbb{R}^{3}$ given by

$$
\Phi(x)=\frac{e^{i k|x|}}{4 \pi|x|}, \quad x \neq 0
$$

and where $|$.$| denotes the usual Euclidean norm and k=\omega / c$ is the wave number.

Let us now consider a bounded and connected obstacle $D \subset \mathbb{R}^{3}$ and $U^{i}$ (the solution in free space) the incident wave. We then have an extra condition to be satisfied at the interface between the obstacle and the exterior medium. Therefore we will consider either a Dirichlet, Neumann or Robin boundary condition at the boundary $\Gamma:=\partial D$. In any case, if $D$ has no energy traps we expect the obstacle to give rise to a scattered wave $U^{s}$ and therefore the asymptotic behaviour of the solution is given by

$$
U(x, t):=U^{i}(x, t)+U^{s}(x, t) \approx e^{-i \omega t}\left(\Phi(x-y)+u^{s}(x)\right), \quad t \rightarrow \infty,
$$

and the scattered field $u^{s}$ behaves as an outgoing spherical wave, that is, it satisfies the Sommerfeld radiation condition (see [45])

$$
\lim _{r \rightarrow \infty} r\left(\frac{\partial u^{s}}{\partial r}-i k u^{s}\right)=0 .
$$

The physical meaning of this condition is that there are no energy sources at infinity (see the classical work of Sommerfeld [63]). As the solution $U$ satisfies the wave equation (2.1) in $\mathbb{R}^{m} \backslash \bar{D}$ we get that

$$
\Delta u^{s}+k^{2} u^{s}=0, \quad x \in \mathbb{R}^{m} \backslash \bar{D},
$$


that is, the solution to the Helmholtz equation can be interpreted as the spatial dependence of a time harmonic acoustic wave as $t \rightarrow \infty$. Mathematically, the radiation condition will ensure uniqueness of solution to the Helmholtz equation.

We also note that if the point source $y$ goes to infinity in the direction $-d$, then from the asymptotic behaviour of the fundamental solutions we get

$$
\lim _{r \rightarrow \infty}\left(4 \pi r e^{-i k r} \Phi(x+r d)\right)=e^{i k x . d}
$$

so in this case it makes sense to approximate the point source by an incident plane field $u^{i}(x)=e^{i k x . d}$ up to some multiplicative constant depending on the distance $r$ between the evaluation point $x$ to the source point $y$. We also note that defining the total field $u=u^{i}+u^{s}$ as the sum of the incident and scattered fields, the boundary conditions on $\Gamma$ carry over from the total wave $U$ to the total field $u$. This means that if for instance we consider a sound-soft obstacle $D$, that is, the pressure of the total wave vanishes at the boundary $\Gamma$ of $D$, then the boundary condition imposed is

$$
U(x, t)=0, \quad x \in \Gamma, t \geq 0,
$$

which implies the Dirichlet boundary condition for $u$ given by

$$
u(x)=0, \quad x \in \Gamma
$$

since from (2.3)

$$
U(x, t) \approx u(x) e^{-i \omega t}, \quad t \rightarrow \infty
$$

where again $u=u^{i}+u^{s}$. In the same way, for sound-hard obstacles, the normal velocity vanishes on the boundary $\Gamma$ and so we get the Neumann boundary condition for $u$

$$
\frac{\partial u(x)}{\partial \nu}=0, \quad x \in \Gamma
$$

where $\nu$ is the exterior unit normal to $D$. Since there are no perfect sound-soft or soundhard obstacles in reality, a more realistic situation is the one where the pressure and the normal velocity are proportional at the boundary, that is, an impedance boundary condition

$$
\frac{\partial u(x)}{\partial \nu}+i \lambda(x) u(x)=0, \quad x \in \Gamma,
$$

with $\lambda \geq 0$. All these three cases will be addressed during this work.

\subsection{The Direct Acoustic Scattering Problem}

The main topic of this work is a method to numerically solve the inverse acoustic scattering problem. Therefore, a solid knowledge on the direct problem is needed. In this 
section we present the basic results on the solutions to the Helmholtz equation and to the direct problem, that will be of crucial importance later on when studying the inverse problem.

We are interested in time harmonic acoustic obstacle scattering. Therefore, as motivated in the previous section, we want to find a solution to the following problem:

Direct Problem 2.1. Given an open obstacle $D$ of class $C^{2}$ with connected boundary $\Gamma$ and an incident field $u^{i}$ we want to find the scattered field $u^{s} \in C^{2}\left(\mathbb{R}^{m} \backslash \bar{D}\right) \cap C\left(\mathbb{R}^{m} \backslash D\right)$, for $m=2,3$ that satisfies

$$
\begin{array}{r}
\Delta u^{s}+k^{2} u^{s}=0, \quad x \in \mathbb{R}^{m} \backslash \bar{D} \\
B u=0, \quad x \in \Gamma:=\partial D, \\
\lim _{r \rightarrow \infty} r^{\frac{m-1}{2}}\left(\frac{\partial u^{s}}{\partial r}-i k u^{s}\right)=0,
\end{array}
$$

where the total field $u$ is given by the sum of the incident field $u^{i}$ and the scattered field $u^{s}$, that is, $u=u^{i}+u^{s}$.

A solution satisfying the Sommerfeld radiation condition (2.7) is called a radiating solution. Again we stress the notation $\Gamma$ for the boundary of $D$, that will be carried out throughout this work. The differential operator $B$ represents one of the already referred boundary conditions, that is,

$$
\begin{aligned}
B u=\left.u\right|_{\Gamma} & \text { (Dirichlet); } \\
B u=\left.\left(\frac{\partial u}{\partial \nu}\right)\right|_{\Gamma} \quad & \text { (Neumann); } \\
B u=\left.\left(\frac{\partial u}{\partial \nu}+i \lambda u\right)\right|_{\Gamma} & \text { (Robin) }
\end{aligned}
$$

where $\lambda \geq 0$ is a continuous function defined on $\Gamma$ and $\nu$ is the exterior unit normal to $D$. All these boundary conditions are to be satisfied in the sense of uniform convergence on $\Gamma$. We note that the Neumann case is a particular case of the Robin case for $\lambda=0$ and that the Dirichlet case can be seen as the limit of the Robin case as $\lambda \rightarrow \infty$.

Most results presented in this section on the properties of the solutions to the direct problem have as primary tools the following first and second Green's theorems.

Theorem 2.2 (Green's Theorem). Let $D$ be a domain of class $C^{1}$. Then for $u \in C^{1}(\bar{D})$ and $v \in C^{2}(\bar{D})$ we have the first Green's theorem

$$
\int_{D}(u \Delta v+\operatorname{grad} u \cdot \operatorname{grad} v) d x=\int_{\partial D} u \frac{\partial v}{\partial \nu} d s .
$$

Moreover if $u, v \in C^{2}(\bar{D})$ we have the second Green's theorem

$$
\int_{D}(u \Delta v-v \Delta u) d x=\int_{\partial D}\left(u \frac{\partial v}{\partial \nu}-v \frac{\partial u}{\partial \nu}\right) d s .
$$


Proof. The first theorem is proved by applying the divergence theorem to the vector field $(u \operatorname{grad} v) \in C^{1}(\bar{D})$. The second is obtained by interchanging the roles of $u$ and $v$ in the first and subtracting both equations.

Based on these theorems, a classical result for representing the solution can be achieved. For its formulation we will need the fundamental solution to the Helmholtz equation in $\mathbb{R}^{m}$ given by

$$
\Phi(x)= \begin{cases}\frac{i}{4} H_{0}^{(1)}(k|x|), & m=2 \\ \frac{e^{i k|x|}}{4 \pi|x|}, & m=3\end{cases}
$$

where $H_{0}^{(1)}$ is the Hankel function of first kind and order zero given by

$$
H_{0}^{(1)}(t)=J_{0}(t)+i Y_{0}(t), \quad t \in \mathbb{R},
$$

where the Bessel function of order zero $J_{0}$ is analytic for all $t \in \mathbb{R}$ and the Neumann function of order zero has a logarithmic singularity at $t=0$ (e.g [9, Chap. 3.4.] for details). Therefore both the fundamental solutions have singularities at zero, that will need to be taken care of for numerical purposes. We will define

$$
\Phi(x, y):=\Phi(|x-y|)
$$

to simplify the notation.

We are now in position to present the classical Green's representation formula for exterior radiating solutions to the Helmholtz equation.

Theorem 2.3 (Green's Representation Formula). Assume the bounded set $D \subset \mathbb{R}^{m}$ to be the open complement of an unbounded domain of class $C^{2}$.

Let $u^{s} \in C^{2}\left(\mathbb{R}^{m} \backslash \bar{D}\right) \cap C\left(\mathbb{R}^{m} \backslash D\right)$ be a radiating solution to the Helmholtz equation (2.5) which possesses a normal derivative on the boundary in the sense that the limit

$$
\frac{\partial u^{s}}{\partial \nu}(x)=\lim _{h \rightarrow 0^{+}} \nu(x) \cdot \operatorname{grad} u^{s}(x+h \nu(x)), \quad x \in \Gamma,
$$

exists uniformly on $\Gamma$. Then we have Green's representation formula

$$
u^{s}(x)=\int_{\Gamma}\left(u^{s}(y) \frac{\partial \Phi(x, y)}{\partial \nu(y)}-\frac{\partial u^{s}}{\partial \nu}(y) \Phi(x, y)\right) d s(y), \quad x \in \mathbb{R}^{m} \backslash \bar{D} .
$$

Proof. We will just state a sketch of the proof and refer for details to [9. Sec. 3.4.] for the two-dimensional case $m=2$ and [9, Thm. 2.4.] for the three-dimensional case $m=3$.

Denoting by $B(x, r)$ the ball with center in $x$ and radius $r$, let us then define the set $G^{*}=B(0, R) \backslash(\overline{D \cup B(x, r)})$ with $x \in \mathbb{R}^{m} \backslash \bar{D}$ and with $R$ sufficiently large and $r$ 
sufficiently small such that $D \subset B(0, R), B(x, r) \subset B(0, R)$ and $B(x, r) \cap D=\emptyset$. As $u^{s}$ and $\Phi$ satisfy the Helmholtz equation in $G^{*}$ we have that

$$
\int_{G^{*}}\left(u^{s}(y) \Delta_{y} \Phi(x, y)-\Phi(x, y) \Delta u^{s}(y)\right) d y=0 .
$$

By the definition of the fundamental solution, we have

$$
\lim _{r \rightarrow 0} \int_{\partial B(x, r)}\left(u^{s}(y) \frac{\partial \Phi(x, y)}{\partial \nu(y)}-\Phi(x, y) \frac{\partial u^{s}}{\partial \nu(y)}(y)\right) d s(y)=u^{s}(x),
$$

where $\nu$ is the exterior unit normal to $G^{*}$ and therefore the interior unit normal to $B(x, r)$. By the radiation condition one can also prove that

$$
\lim _{R \rightarrow \infty} \int_{\partial B(0, R)}\left(u^{s}(y) \frac{\partial \Phi(x, y)}{\partial \nu(y)}-\Phi(x, y) \frac{\partial u^{s}}{\partial \nu(y)}(y)\right) d s(y)=0 .
$$

The proof is now complete by applying Green's theorem to $u=u^{s}$ and $v=\Phi(x,$.$) on G^{*}$ and let $r \rightarrow 0$ and $R \rightarrow \infty$.

From the previous representation one can conclude that if $u$ is a $C^{2}$-solution to the Helmholtz equation in $\mathbb{R}^{m} \backslash \bar{D}$ then $u$ is analytic in $\mathbb{R}^{m} \backslash \bar{D}$ (see [9, thm.2.2]).

We now introduce the notation $\Omega_{m}$ for the unit spherical surface in $\mathbb{R}^{m}$, that is,

$$
\Omega_{m}=\left\{x \in \mathbb{R}^{m}:|x|=1\right\}
$$

where as before |.| denotes the usual Euclidean norm.

From the previous theorem one can conclude the following asymptotic behaviour of the solution.

Theorem 2.4 (Far-field pattern). Every radiating solution $u^{s}$ to the Helmholtz equation in $\mathbb{R}^{m} \backslash \bar{D}$ has an asymptotic behaviour of an outgoing spherical wave

$$
u^{s}(x)=\frac{e^{i k|x|}}{|x|^{\frac{m-1}{2}}}\left(u_{\infty}(\hat{x})+O\left(\frac{1}{|x|}\right)\right), \quad|x| \rightarrow \infty
$$

uniformly in all directions $\hat{x}=x /|x| \in \Omega_{m}$ where the function $u_{\infty}$ is called the far-field pattern of $u$. Under the assumptions of theorem 2.3 we have

$$
u_{\infty}(\hat{x})=\varrho_{m} \int_{\Gamma}\left(u^{s}(y) \frac{\partial e^{-i k \hat{x} \cdot y}}{\partial \nu(y)}-\frac{\partial u^{s}}{\partial \nu}(y) e^{-i k \hat{x} \cdot y}\right) d s(y)
$$

where

$$
\varrho_{m}= \begin{cases}\frac{e^{i \pi / 4}}{\sqrt{8 \pi k}}, & m=2 \\ \frac{1}{4 \pi}, & m=3\end{cases}
$$


Proof. Using the Taylor expansion of the square root function around 1, we get that

$$
\begin{aligned}
|x-y| & =\sqrt{|x|^{2}-2 x \cdot y+|y|^{2}} \\
& =|x| \sqrt{1-2 \frac{\hat{x} \cdot y}{|x|}+\frac{|y|^{2}}{|x|^{2}}} \\
& =|x|-\hat{x} \cdot y+O\left(\frac{1}{|x|}\right)
\end{aligned}
$$

as $|x| \rightarrow \infty$ uniformly for $y \in \Gamma$. Therefore we derive

$$
\begin{aligned}
\frac{e^{i k|x-y|}}{|x-y|} & =\frac{e^{i k|x|}}{|x|}\left(e^{-i k \hat{x} \cdot y}+O\left(\frac{1}{|x|}\right)\right) \\
\frac{\partial}{\partial \nu(y)} \frac{e^{i k|x-y|}}{|x-y|} & =\frac{e^{i k|x|}}{|x|}\left(\frac{\partial e^{-i k \hat{x} \cdot y}}{\partial \nu(y)}+O\left(\frac{1}{|x|}\right)\right)
\end{aligned}
$$

as $|x| \rightarrow \infty$ uniformly for $y \in \Gamma$. Replacing this in (2.11) we have the result for $m=3$. For $m=2$, the procedure is similar (see [9, Sec. 3.4.]), using the asymptotics of the Hankel function.

Remark 2.5. We have seen in Section 2.1 that if the source point $y$ is very far in the direction $-d$ from the obstacle, then the point source incident field can be approximated (up to a multiplicative constant depending on the distance $|x-y|$ ) by a plane wave $u^{i}(x)=e^{i k x \cdot d}$, with $d \in \Omega_{m}$. In other words, the asymptotic behaviour (2.4) means that the far-field of a point source is a plane wave, that is,

$$
\Phi_{\infty}(x ; y)=\rho_{m} e^{i k x \cdot d} .
$$

In the same way, by theorem 2.4 if one measures the scattered wave very far from the obstacle, one can assume that the measured data is the far-field pattern (up to the same multiplicative constant). Both this assumptions will be taken later on for the inverse problem, where we will consider an incident plane wave and the far-field pattern as data.

In this way we will present a few more properties of the far-field pattern, since it will be important in the forthcoming chapters. From the representation (2.13) we see that the far-field pattern $u_{\infty}$ is analytic on $\Omega_{m}$. The following result shows us that having an incident field in the direction $d$ and measuring the far-field pattern in the direction $\hat{x}$ is the same as having an incident field in the direction $-\hat{x}$ and measuring the far-field pattern in the direction $-d$, that is, at large distances from it, the obstacle as mirroring properties.

Theorem 2.6 (Reciprocity relation). For any of the boundary conditions previously mentioned (2.8)-(2.10), we have that the far-field pattern satisfies

$$
u_{\infty}(\hat{x} ; d)=u_{\infty}(-d ;-\hat{x}), \quad \hat{x}, d \in \Omega_{m}
$$


where $u_{\infty}(. ; d)$ denotes the far-field pattern obtained by scattering of a plane wave with incident direction $d \in \Omega_{m}$.

Proof. Making use of the fact that the incident plane field $u^{i}(x ; d)=e^{i k x \cdot d}$ satisfies the Helmholtz equation inside the obstacle $D$, by the second Green's theorem applied to $u=u^{i}(. ; d)$ and $v=u^{i}(. ;-\hat{x})$ we get

$$
\int_{\Gamma}\left(u^{i}(. ; d) \frac{\partial u^{i}(. ;-\hat{x})}{\partial \nu}-u^{i}(. ;-\hat{x}) \frac{\partial u^{i}(. ; d)}{\partial \nu}\right) d s=0 .
$$

Applying the same tools and procedure for the scattered wave in the exterior domain, making use of the radiation condition we get

$$
\int_{\Gamma}\left(u^{s}(. ; d) \frac{\partial u^{s}(. ;-\hat{x})}{\partial \nu}-u^{s}(. ;-\hat{x}) \frac{\partial u^{s}(. ; d)}{\partial \nu}\right) d s=0 .
$$

From (2.13) we get

$$
\frac{1}{\varrho_{m}} u_{\infty}(\hat{x} ; d)=\int_{\Gamma}\left(u^{s}(. ; d) \frac{\partial u^{i}(. ;-\hat{x})}{\partial \nu}-u^{i}(. ;-\hat{x}) \frac{\partial u^{s}(. ; d)}{\partial \nu}\right) d s
$$

and interchanging the roles of $d$ and $\hat{x}$

$$
\frac{1}{\varrho_{m}} u_{\infty}(-d ;-\hat{x})=\int_{\Gamma}\left(u^{s}(. ;-\hat{x}) \frac{\partial u^{i}(. ; d)}{\partial \nu}-u^{i}(. ; d) \frac{\partial u^{s}(. ;-\hat{x})}{\partial \nu}\right) d s .
$$

Subtracting the last equation from the sum of the previous three, one gets

$$
\frac{1}{\varrho_{m}}\left(u_{\infty}(\hat{x} ; d)-u_{\infty}(-d ;-\hat{x})\right)=\int_{\Gamma}\left(u(. ; d) \frac{\partial u(. ;-\hat{x})}{\partial \nu}-u(. ;-\hat{x}) \frac{\partial u(. ; d)}{\partial \nu}\right) d s .
$$

Making use of the boundary condition $B u(. ; d)=B u(. ;-\hat{x})=0$, for any $B$ defined in (2.8)-(2.10), the left hand side of the previous equation vanishes and we get the result.

The question whether the far-field pattern $u_{\infty}$ uniquely determines the scattered field $u^{s}$ is affirmatively answered by Rellich's Lemma. We refer to [9, thm.2.11] for the proof.

Lemma 2.7 (Rellich). Let $D$ be as in theorem 2.3 and $u \in C^{2}\left(\mathbb{R}^{m} \backslash \bar{D}\right)$ be a solution to the Helmholtz equation satisfying

$$
\lim _{r \rightarrow \infty} \int_{\{|x|=r\}}|u|^{2} d s=0 .
$$

Then $u=0$ in $\mathbb{R}^{m} \backslash \bar{D}$. 
We formulate now the result that establishes the promised unique relation between the far-field pattern and the scattered field as a corollary of the previous result and the asymptotic behaviour (2.12) of the scattered field (e.g. [9, thm. 2.13]).

Corollary 2.8. Let $D$ be as in theorem 2.3 and $u \in C^{2}\left(\mathbb{R}^{m} \backslash \bar{D}\right), m=2,3$, be a radiating solution to the Helmholtz equation for which the far-field pattern $u_{\infty}$ vanishes on $\Omega_{m}$. Then $u=0$ in $\mathbb{R}^{m} \backslash \bar{D}$.

\subsubsection{Layer Potentials}

In this section we will present the layer potentials and basic results on their properties. The layer potentials will be of crucial importance to represent the solution to the direct problem and later on for the first step of the hybrid method to numerically solve the inverse problem.

Given an integrable function $\varphi$, the single-layer potential is defined by

$$
w(x)=\int_{\Gamma} \Phi(x, y) \varphi(y) d s(y), \quad x \in \mathbb{R}^{m} \backslash \bar{D}
$$

while the double-layer potential is defined by

$$
v(x)=\int_{\Gamma} \frac{\partial \Phi(x, y)}{\partial \nu(y)} \varphi(y) d s(y), \quad x \in \mathbb{R}^{m} \backslash \bar{D} .
$$

Explicit computations show that both are solutions to the Helmholtz equation in $D$ and in $\mathbb{R}^{m} \backslash \bar{D}$ and that they satisfy the Sommerfeld radiation condition. Green's representation theorem 2.3 tells us that any solution to the Helmholtz equation can be represented as a combination of single-and double-layer potentials. We will now state the classical result on the jump relations of these potentials, but similar results can also be shown for densities $\varphi$ living in Sobolev spaces (see [27]).

Theorem 2.9. Let $\Gamma$ be of class $C^{2}$ and let $\varphi$ be continuous. Then the single-layer potential $w$ with density $\varphi$ is continuous throughout $\mathbb{R}^{m}$ and satisfies the estimate in the usual maximum norm

$$
\|w\|_{\infty, \mathbb{R}^{m}} \leq C\|\varphi\|_{\infty, \Gamma}
$$

for some constant $C$ depending on $\Gamma$. On the boundary we have the representations

$$
\begin{aligned}
w(x) & =\int_{\Gamma} \Phi(x, y) \varphi(y) d s(y), \quad x \in \Gamma, \\
\frac{\partial w_{ \pm}}{\partial \nu}(x) & =\mp \frac{\varphi(x)}{2}+\int_{\Gamma} \frac{\partial \Phi(x, y)}{\partial \nu(x)} \varphi(y) d s(y), \quad x \in \Gamma,
\end{aligned}
$$


where

$$
\frac{\partial w_{ \pm}}{\partial \nu}(x):=\lim _{h \rightarrow 0^{+}} \nu(x) \cdot \operatorname{grad} w(x \pm h \nu(x)), \quad x \in \Gamma,
$$

is to be understood in the sense of uniform convergence on $\Gamma$ and where the integrals exist as improper integrals.

The double-layer potential $v$ with density $\varphi$ can be continuosly extended from $D$ to $\bar{D}$ and from $\mathbb{R}^{m} \backslash \bar{D}$ to $\mathbb{R}^{m} \backslash D$ with limiting values

$$
v_{ \pm}(x)= \pm \frac{\varphi(x)}{2}+\int_{\Gamma} \frac{\partial \Phi(x, y)}{\partial \nu(y)} \varphi(y) d s(y), \quad x \in \Gamma,
$$

where

$$
v_{ \pm}(x):=\lim _{h \rightarrow 0^{+}} v(x \pm h \nu(x)), \quad x \in \Gamma
$$

and the integral exists as an improper integral. We also have the estimate

$$
\|v\|_{\infty, \bar{D}} \leq C\|\varphi\|_{\infty, \Gamma}, \quad\|v\|_{\infty, \mathbb{R}^{m} \backslash D} \leq C\|\varphi\|_{\infty, \Gamma},
$$

for some constant $C$ depending on $\Gamma$. The normal derivative has no jump in the sense that

$$
\lim _{h \rightarrow 0^{+}}\left(\frac{\partial v}{\partial \nu}(x+h \nu(x))-\frac{\partial v}{\partial \nu}(x-h \nu(x))\right)=0, \quad x \in \Gamma,
$$

uniformly in $\Gamma$.

Proof. We refer to theorems 2.12, 2.16, 2.17, and 2.23 in ([8]).

Let us now introduce the single-layer operator $S$ given by

$$
(S \varphi)(x):=\int_{\Gamma} \Phi(x, y) \varphi(y) d s(y), \quad x \in \Gamma
$$

and the double-layer operator $K$ given by

$$
(K \varphi)(x):=\int_{\Gamma} \frac{\partial \Phi(x, y)}{\partial \nu(y)} \varphi(y) d s(y), \quad x \in \Gamma .
$$

as well as the normal derivative operators

$$
\begin{aligned}
\left(K^{*} \varphi\right)(x) & :=\int_{\Gamma} \frac{\partial \Phi(x, y)}{\partial \nu(x)} \varphi(y) d s(y), \quad x \in \Gamma \\
(T \varphi)(x) & :=\frac{\partial}{\partial \nu(x)} \int_{\Gamma} \frac{\partial \Phi(x, y)}{\partial \nu(y)} \varphi(y) d s(y), \quad x \in \Gamma .
\end{aligned}
$$


The previous jump relations can be given in terms of these operators, namely through

$$
\begin{aligned}
w(x)=(S \varphi)(y), & \frac{\partial w_{ \pm}}{\partial \nu}(x)=\mp \frac{\varphi(x)}{2}+\left(K^{*} \varphi\right)(x), \\
v_{ \pm}(x)= \pm \frac{\varphi(x)}{2}+K \varphi(x), & \frac{\partial v}{\partial \nu}(x)=(T \varphi)(x)
\end{aligned}
$$

for $x \in \Gamma$. We will now state some results on the mapping properties of these four operators. For proofs we refer to [8, thm. 2.31].

Theorem 2.10. Let $\Gamma$ be of class $C^{2}$. Then

(a) the operators $S, K$ and $K^{*}$ are bounded from $C(\Gamma)$ into $C^{0, \alpha}(\Gamma)$,

(b) the operators $S$ and $K$ are also bounded from $C^{0, \alpha}(\Gamma)$ into $C^{1, \alpha}(\Gamma)$,

(c) the operator $T$ is bounded from $C^{1, \alpha}(\Gamma)$ into $C^{0, \alpha}(\Gamma)$.

We also state the following theorem for weak solutions and refer to [27] for the proof.

Theorem 2.11. Let $p \in \mathbb{N} \cap\{0\}$ and $\alpha \in(0,1)$.

(a) Let $\Gamma$ be of class $C^{p+2, \alpha}$. Then $S$ and $K$ are bounded from $H^{p}(\Gamma)$ into $H^{p+1}(\Gamma)$ and $T$ is bounded from $H^{p+1}(\Gamma)$ into $H^{p}(\Gamma)$.

(b) Let $\Gamma$ be of class $C^{p+3, \alpha}$. Then $K^{*}$ is bounded from $H^{p}(\Gamma)$ into $H^{p+1}(\Gamma)$.

Similar results can also be obtained in the case that $\Gamma$ is not $C^{2}$-smooth (see [51]).

We also introduce the far-field operators

$$
\begin{aligned}
\left(S_{\infty} \varphi\right)(\hat{x}) & :=\varrho_{m} \int_{\Gamma} e^{-i k \hat{x} \cdot y} \varphi(y) d s(y), \quad \hat{x} \in \Omega \\
\left(K_{\infty} \varphi\right)(\hat{x}) & :=\varrho_{m} \int_{\Gamma} \frac{\partial e^{-i k \hat{x} \cdot y}}{\partial \nu(y)} \varphi(y) d s(y), \quad \hat{x} \in \Omega .
\end{aligned}
$$

with $\varrho_{m}$ given as in (2.14). Since their integral kernels are continuous, the previous operators are compact from the space of continuously $k$-differentiable functions $C^{k}(\gamma)$ into $C^{k}\left(\Omega_{m}\right)$ and from the space of Hölder continuously $k$-differentiable functions $C^{k, \alpha}(\gamma)$ for $\alpha>0$ into $C^{k, \alpha}\left(\Omega_{m}\right)$. By the asymptotics of the layer potentials, one can also prove (see [9]) that the far-field pattern of the single layer potential (2.16) is given by

$$
w_{\infty}(\hat{x})=\left(S_{\infty} \varphi\right)(\hat{x}), \quad \hat{x} \in \Omega,
$$

and the far-field of the double-layer potential (2.17) is given by

$$
v_{\infty}(\hat{x})=\left(K_{\infty} \varphi\right)(\hat{x}), \quad \hat{x} \in \Omega .
$$


For a combined single-and double-layer potential

$$
u(x)=\int_{\Gamma}\left(\frac{\partial \Phi(x, y)}{\partial \nu(y)}-i \eta \Phi(x, y)\right) \varphi(y) d s(y), \quad x \in \mathbb{R}^{m} \backslash \Gamma
$$

we would obviously get the far-field given by

$$
u_{\infty}(\hat{x})=\left(\left(K_{\infty}-i \eta S_{\infty}\right) \varphi\right)(x), \quad \hat{x} \in \Omega .
$$

\subsubsection{Uniqueness and Existence theorems}

We recall that the solution $u^{s}$ must satisfy

$$
\begin{array}{r}
\Delta u^{s}+k^{2} u^{s}=0, \quad x \in \mathbb{R}^{m} \backslash \bar{D}, \\
B u^{s}=f, \quad x \in \Gamma:=\partial D, \\
\lim _{r \rightarrow \infty} r^{\frac{m-1}{2}}\left(\frac{\partial u^{s}}{\partial r}-i k u^{s}\right)=0 .
\end{array}
$$

We consider $f:=-B u^{i}$, where the incident field $u^{i}$ is considered to be analytic up to the boundary of $D$.

We will state the uniqueness and existence results concerning the three boundary conditions (2.8)-(2.10) but the proofs will only given for some of the cases. We refer to $[8,9]$ for the remaining ones.

Theorem 2.12 (Uniqueness). The exterior Dirichlet, Neumann or Robin problems have at most one solution.

Proof. We will just give a sketch of the proof for the Dirichlet and Neumann case. For details see [9, Thm.3.7.]. For the Robin case we refer to [8, Thm.3.37.].

One has to show that solutions to the homogeneous boundary value problem $B u^{s}=0$ vanish on the domain of definition. From the radiation condition and applying Green's theorem one concludes that

$$
\lim _{r \rightarrow \infty} \int_{\Omega_{r}}\left(\left|\frac{\partial u^{s}}{\partial \nu}\right|^{2}+k^{2}|u|^{2}\right) d s=-2 k \operatorname{Im} \int_{\Gamma} u^{s} \frac{\partial \overline{u^{s}}}{\partial \nu} d s
$$

where $\Omega_{r}=\{x:|x|=r\}$. As $u^{s}$ is just assumed to be continuous up to the boundary, for the Dirichlet case the existence of the integral on the right-hand side must be assured. We overcome the problem by considering $\Gamma$ of class $C^{2}$ and $u^{i}$ to be at least $C^{1, \alpha}$ (see [47]). By the boundary conditions $u^{s}=0$ or $\partial u^{s} / \partial \nu=0$ on $\Gamma$ we get that

$$
\lim _{r \rightarrow \infty} \int_{\Omega_{r}}|u|^{2} d s=0
$$

and by Rellich's lemma 2.7 one gets the result. 
Theorem 2.13 (Existence). There exists a unique solution to the exterior Dirichlet, Neumann or Robin problem.

Proof. We first consider the Dirichlet boundary condition. We start by writing a candidate for the solution as a combined single-and double-layer potential representation, that is, let

$$
v(x):=((K-i \eta S) \varphi)(x), \quad x \in \mathbb{R}^{m} \backslash \bar{D} .
$$

with some coupling parameter $\eta>0$. By the properties of the layer potentials, we conclude that $v$ satisfies the Helmholtz equation and the radiation condition. By the jump relations, in order to fulfil the boundary equation, we get that

$$
\frac{\varphi}{2}+(K-i \eta S) \varphi=f
$$

must be satisfied over $\Gamma$. The fact that $S$ and $K$ are compact operators from $C(\Gamma)$ into itself (thm. 2.10 combined with the compact embedding of $C^{0, \alpha}(\Gamma)$ in $C(\Gamma)$ ) and the Fredholm-Riesz theory for equations of the second kind with a compact operator show that the equation has a solution if the operator $I+2(K-i \eta S)$ is injective. Let us then assume that $\varphi$ is a solution to the homogeneous equation

$$
\varphi+2(K-i \eta S) \varphi=0 .
$$

Then the potential $v$ given by (2.25) satisfies the exterior homogeneous boundary condition and by uniqueness of this problem we conclude that $v=0$ on $\mathbb{R}^{m} \backslash \bar{D}$. The jump relations from thm. 2.9 yield

$$
v_{-}=-\varphi, \quad \frac{\partial v_{-}}{\partial \nu}=-i \eta \varphi \quad \text { on } \Gamma
$$

and from the first Green's theorem applied to $v_{-}$and $\bar{v}_{-}$in $D$ we get

$$
i \eta \int_{\Gamma}|\varphi|^{2} d s=\int_{\Gamma} \bar{v}_{-} \frac{\partial v_{-}}{\partial \nu} d s=\int_{D}\left(|\operatorname{grad} v|^{2}-k^{2}|v|^{2}\right) .
$$

Taking the imaginary part of the previous equation we get $\varphi=0$ and the existence proof is finished.

For the Neumann and Robin cases the proofs go in a similar way, choosing appropriate combinations of layer potentials. We refer to $[9$, thm.3.10] and $[8$, thm.3.38], respectively, for details.

Remark 2.14. The estimates of theorem 2.9, along with the continuous dependence of the density $\varphi$ on the boundary data $f$ contained in the previous proof as a consequence of the Fredholm-Riesz theory, show continuous dependence of the solution $u^{s}$ on the boundary data $f$. 


\subsection{The Inverse Acoustic Scattering Problem}

The inverse problem is a much harder and more exciting problem to solve. It has been studied for the last decades but there are still many rather fundamental open problems, namely uniqueness proofs for a finite number of incident waves. In this section we will give an overview of the results for the time-harmonic acoustic obstacle scattering problem under consideration.

The formulation of the inverse problem we want to solve is the following:

Inverse Problem 2.15. Let $u^{i}$ be an incident field, usually considered to be a plane wave $u^{i}(x)=e^{i k x \cdot d}$, with incident direction $d$ such that $|d|=1$.

Given a far-field pattern $u_{\infty}$ corresponding to a scattered field $u^{s}$ satisfying

$$
\begin{gathered}
\Delta u^{s}+k^{2} u^{s}=0, \quad x \in \mathbb{R}^{m} \backslash \bar{D}, \\
B\left(u^{i}+u^{s}\right)=0, \quad x \in \Gamma:=\partial D, \\
\lim _{r \rightarrow \infty} r^{\frac{m-1}{2}}\left(\frac{\partial u^{s}}{\partial r}-i k u^{s}\right)=0,
\end{gathered}
$$

where $B$ is known and is one of the operators (2.8)-(2.10) corresponding to a Dirichlet, Neumann or Robin boundary condition, find the position and shape of the obstacle $D$ of class $C^{2}$. In the case of the impedance boundary condition (2.10) we also want to find the unknown impedance $\lambda$.

Remark 2.16. The latter case is equivalent to recovering the obstacle and the boundary condition, since we recover also the unknown impedance $\lambda$. As referred before, both the Dirichlet and Neumann are particular cases of the Robin one. If $\lambda$ is close to zero we recover the information that the obstacle is sound-hard and if $\lambda$ is large that it is soundsoft. A coated-obstacle can also be reconstructed though we assume $\lambda$ to be a continuous function.

The problem 2.15 is ill-posed in the sense of Hadamard [17] and is also non-linear. The ill-posedness comes from the fact that the determination of $D$ does not depend continuously on the far-field pattern $u_{\infty}$. In the procedure of the hybrid method (as explained later on in section 3.2), this is illustrated in the reconstruction of $u^{s}$ from the knowledge of $u_{\infty}$, since it can be seen as the inversion of the integral operator (2.24) which is a compact operator due to its continuous kernel. The non-linearity comes from the fact that the scattered wave does not depend linearly on the obstacle. This can be illustrated as finding the position of the obstacle as the location of the zero level set of $B u$ not being a linear problem. Moreover, scattering by two different obstacles is different from the sum of scattering by each one of them separately.

The first and only issue that needs to be addressed is uniqueness. Note that existence is a wrong issue to study since we assume that the given far-field $u_{\infty}$ corresponds to scattering by the unknown obstacle $D$. In this sense, existence is settled. In fact, if the 
far-field data contains noise then existence is not a matter to ask for, since the problem is severely ill-posed and a solution to the noisy data could be very far from the true solution. Moreover, if one actually wanted to proof existence of a solution for a general given far-field pattern $u_{\infty}$, it would imply that one would be able to characterize whether the zero level set of the scattered field corresponding to the given far-field $u_{\infty}$ is a close curve, which is now-a-days way beyond the capabilities of the available theory. Therefore we will proceed by presenting some classical results on the uniqueness of this inverse problem and sketch the proofs. We start by the classical result presented in [45] based on the ideas of Schiffer.

Theorem 2.17. Assume that $D_{1}$ and $D_{2}$ are two sound-soft scatterers such that the farfield patterns coincide for an infinite number of incident plane waves with distinct directions and one fixed wave number. Then $D_{1}=D_{2}$.

Proof. Let $u_{j}^{s}(., d), j=1,2$, be the scattered field corresponding to scattering by the obstacle $D_{j}$ with incident direction $d$ and let $u_{j}(., d), j=1,2$, be the corresponding total field. By thm. 2.8 we know that the far-field pattern uniquely determines the scattered wave and so we have that $u_{1}^{s}(x, d)=u_{2}^{s}(x, d)$ for $x \in G$ where $G$ is the unbounded component of $\mathbb{R}^{m} \backslash\left(D_{1} \cup D_{2}\right)$. Consequently, we get that $u_{1}(x, d)=u_{2}(x, d)$ for $x \in G$ and by the boundary condition and continuity of the total fields we get that $u_{1}(x, d)=u_{2}(x, d)=0$ for $x \in \partial G$. We now assume that $D_{1} \neq D_{2}$ in order to obtain a contradiction. Without loss of generality we can assume that $D^{*}=\left(\mathbb{R}^{m} \backslash G\right) \backslash \bar{D}_{2}$ is non-empty. Then $u_{2}^{s}(., d)$ is defined in $D^{*}$ since it is describes scattering by $D_{2}$. Therefore $u_{2}(., d)$ satisfies the Helmholtz equation in $D^{*}$ as well as the homogeneous Dirichlet boundary condition on $\partial D^{*}$. Therefore $u_{2}(., d)$ is a Dirichlet eigenfunction of the negative Laplacian in $D^{*}$ with eigenvalue $k^{2}$. In this way, considering an infinite number of incident directions $\left\{d_{n}\right\}, n \in \mathbb{N}$, we have an infinite number of Dirichlet eigenfunctions $u_{2}\left(., d_{n}\right)$ in $D^{*}$ for the same eigenvalue $k^{2}$. The proof is now finished by showing that the $u_{2}\left(., d_{n}\right) \in H_{0}^{1}\left(D^{*}\right)$ (e.g. [9, Lem.3.8]) are linearly independent and that for a fixed eigenvalue there exists only finitely many linearly independent Dirichlet eigenvalues in $H_{0}^{1}\left(D^{*}\right)$ (e.g. [9, proof of thm.5.1]). In this way we get the desired contradiction and the proof is finished.

In this work we are interested in a method to solve the inverse obstacle scattering problem considering as data the far-field pattern for just one incident field. In this way, we will proceed by presenting uniqueness results considering just a finite number of incident directions, namely using some $a$ priori bound on the size of the obstacle. The bound on the size of the obstacle was initially proposed by Colton and Sleeman [11] and recently improved by Gintides [14]. 
Theorem 2.18. Let $D_{1}, D_{2} \in \mathbb{R}^{3}$ be two scatterers which are contained in a ball of radius $R$, let

$$
N:=\sum_{t_{n l}<k R}(2 n+1)
$$

where $t_{n l}, l \in \mathbb{N}$ are the positive zeros of the spherical Bessel function $j_{n}, n \in \mathbb{N}$ and let

$$
M:= \begin{cases}N / 2+1, & N \text { is even } \\ (N+1) / 2, & N \text { is } \text { odd }\end{cases}
$$

Assume also that the far-field patterns for both obstacles coincide for one fixed wave number $k$ and for $M$ different incident directions $d_{n}, n=1, \ldots, M$, such that $d_{n} \neq \pm d_{n^{\prime}}$ for $n \neq n^{\prime}$. Then $D_{1}=D_{2}$.

Proof. We use the same definition of $D^{*}$ as used in the previous proof. The Courant maximum-minimum principle for compact symmetric operators implies that the negative Laplacian Dirichlet eigenvalues have the following property (see [46, thm.4.7]): The $n$-th eigenvalue $\lambda_{n}$ ordered by magnitude taking into account its multiplicity for a ball $B$ containing the domains $D_{1}$ and $D_{2}$ is always smaller than the $n$-th eigenvalue $\mu_{n}$ for the subdomain $D^{*} \subset B$. In particular, for $\lambda_{n}=k^{2}$, the multiplicity of $\lambda_{n}$ must be less than or equal to the sum of multiplicities of the eigenvalues of the ball $B$ that are smaller than $k^{2}$. It is known that the eigenvalues of the ball $B$ are given by $\mu_{n l}=t_{n l}^{2} / R^{2}$ (e.g. [9. pp.57]) and each has multiplicity $2 n+1$ (e.g. [9, thm.2.6]). Therefore the multiplicity of $\lambda_{n}$ must be smaller or equal to $N$, by definition of $N$. Assuming $D^{*}$ is non-empty, that is, that $D_{1} \neq D_{2}$, in the same way as in the previous proof we will be led to a contradition. We have that $M$ incident directions $\left\{d_{n}\right\}, n=1, \ldots, M$, would lead to $M$ linearly independent eigenfunctions $u_{2}\left(., d_{n}\right), n=1, \ldots, M$ with the same eigenvalue $k^{2}$. Moreover, under the assumptions on the incident directions, the conjugate complex total fields $\bar{u}_{2}\left(., d_{n}\right), n=1, \ldots, M$ would be also linearly independent from the previous and would also satisfy the Laplace equation in $D^{*}$ and the homogeneous Dirichlet boundary condition on $\partial D^{*}$ (see[14]). Therefore we would have $2 M$ linearly independent eigenfunctions related with the eigenvalue $\lambda_{n}$ and so the multiplicity of $\lambda_{n}$ is greater or equal to $2 M$, which leads to a contradiction because $2 M>N$. Therefore $D_{1}=D_{2}$.

Corollary 2.19. Let $D_{1}, D_{2} \in \mathbb{R}^{3}$ be two scatterers which are contained in a ball of radius $R$ such that $k R<t_{10} \approx 4.4939$. Assume also that the far-field patterns coincide for one incident direction. Then $D_{1}=D_{2}$.

Proof. From $k R<t_{10}$ and the fact that $t_{00}$ is the only positive zero $t_{n l}$ of a Bessel spherical function $j_{n}$ satisfying $t_{n l}<t_{10}$, we conclude that $N \leq 1$ and so by the previous theorem one incident direction is enough to uniquely determine the obstacle.

Remark 2.20. A similar result can be obtained in $\mathbb{R}^{2}$ with the restriction $k R<z_{10}$, where $z_{n l}$ are the positive zeros of the Bessel functions $J_{n}$. The proof is identical and 
relies on the fact that $J_{n}(k r) e^{ \pm i k \phi}$ is an entire solution to the Helmholtz equation in $\mathbb{R}^{2}$ and therefore $\mu_{n l}=z_{n l}^{2} / R^{2}$ are Dirichlet eigenvalues on a circle of radius $R$. Everything then follows in a similar way.

Note that for the previous uniqueness results no regularity assumption was made on the domains.

For the Neumann and Robin boundary conditions, the same procedure of proof can not be carried out. This is due to the fact that the domain $D^{*}$ defined as in the previous proofs might have corners or even cusps and the finiteness of the dimensions of the eigenspaces of the Laplace operator with boundary conditions requires the boundary to be sufficiently smooth. This cannot be overcome with requiring more regularity or even analyticity on $D_{1}$ and $D_{2}$ since this does not prevent $D^{*}$ to have corners or cusps. A new idea of proof that could be applicable to these two boundary conditions was suggested by Isakov (e.g. [24]), who obtained a contradiction on the value of an integral over some appropriate contour when its length went to zero. A much simpler approach was presented by Kirsch and Kress [33], where this contradiction was achieved in a pointwise sense. Moreover, this procedure of proof can be carried out for any of the referred boundary conditions, as mentioned in remark 2.22. It is the following result.

Theorem 2.21. Assume that $D_{1}$ and $D_{2}$ are two sound-hard scatterers such that the farfield patterns coincide for all incident plane waves with incident directions within an open non-empty subset of $\Omega_{m}$ and one fixed wave number. Then $D_{1}=D_{2}$.

Proof. By reciprocity (2.15) and analyticity of the far-field pattern, we first conclude that the far-field patterns must coincide for all incident directions. Then, as in thm 2.17 we conclude that $u_{1}(., d)=u_{2}(., d)$ on the unbounded component $G$ of $\mathbb{R}^{m} \backslash\left(\bar{D}_{1} \cup \bar{D}_{2}\right)$. Let $x_{0} \in G$ be fixed and consider the two Neumann problems

$$
\begin{array}{rc}
\Delta w_{j}^{s}+k^{2} w_{j}^{s}=0 & \text { in } \mathbb{R}^{m} \backslash \overline{D_{j}}, \\
\frac{\partial w_{j}^{s}}{\partial \nu}=-\frac{\partial \Phi\left(., x_{0}\right)}{\partial \nu} & \text { on } \partial D_{j}
\end{array}
$$

for $j=1,2$. Our first goal is to prove that $w_{1}^{s}=w_{2}^{s}$ in $G$.

To this end, we choose a bounded $C^{2}$-domain $B$ such that $\mathbb{R}^{m} \backslash B$ is connected, the set $\left(\bar{D}_{1} \cup \bar{D}_{2}\right) \subset B, x_{0} \notin B$ and $k^{2}$ is not an interior Dirichlet eigenvalue for $B$, which is possible to achieve with a proper choice of $B$ due to the strong monotonicity properties of the eigenvalues. Then, from the completeness of $\left\{\left.u^{i}(. ; d)\right|_{\partial B}: d \in \Omega_{m}\right\}$ in $L^{2}(\partial B)$ (e.g. $\left[9\right.$, thm.5.5]), there exists a sequence $\left\{v_{n}\right\}$ in $\operatorname{span}\left\{u^{i}(. ; d): d \in \Omega_{m}\right\}$ such that

$$
\left\|v_{n}-\Phi\left(., x_{0}\right)\right\|_{L^{2}(\partial B)} \rightarrow 0, \quad n \rightarrow \infty .
$$

Then as each $v_{n}$ is a solution to the Helmholtz equation and by the assumption that $k^{2}$ is not an eigenvalue for $B$, we can conclude (e.g. [9, thm.5.4]) that

$$
\operatorname{grad} v_{n} \rightarrow \operatorname{grad} \Phi\left(., x_{0}\right), \quad n \rightarrow \infty
$$


uniformly on compact sets of $B$, in particular, in $\bar{D}_{1} \cup \bar{D}_{2}$. Since the $v_{n}$ are combinations of plane waves, from the first paragraph of this proof, the corresponding scattered fields $v_{n, j}^{s}$ for the obstacles $D_{j}, j=1,2$ must coincide in $G$. This implies also that

$$
\frac{\partial v_{n, j}^{s}}{\partial \nu}=-\frac{\partial v_{n}}{\partial \nu} \text { on } \partial D_{j}, \quad j=1,2
$$

so the convergence (2.31) along with the uniqueness and well-posedness of the solution to the exterior Neumann problem show us that

$$
v_{n, j}^{s} \rightarrow w_{j}^{s}, \quad n \rightarrow \infty
$$

uniformly in compact sets of $\mathbb{R}^{m} \backslash \overline{D_{j}}, j=1,2$. Therefore $w_{1}^{s}=w_{2}^{s}$ in $G$, since as already mentioned the fields $v_{n, j}^{s}, j=1,2$ coincide in $G$.

We now assume that $D_{1} \neq D_{2}$ in order to get a contradiction. Without loss of generality, there exists $x^{*} \in \partial D_{1} \backslash \bar{D}_{2}$. We choose $h>0$ sufficiently small such that the sequence

$$
x_{n}:=x^{*}+\frac{h}{n} \nu\left(x^{*}\right), \quad n \in \mathbb{N},
$$

is contained in $G$. We now consider $w_{n, j}^{s}$ as the solutions to the exterior Neumann problems (2.29)-(2.30) with $x_{0}$ replaced by $x_{n}$. We recall that $w_{n, 1}^{s}=w_{n, 2}^{s}=w_{n}^{s}$ in $G$. On the one hand we have that the Neumann boundary data over $\partial D_{2}$ is uniformly bounded with respect to the maximum norm and along with the well-posedness of the exterior Neumann problem we have that

$$
\left|\frac{\partial w_{n}^{s}}{\partial \nu}\left(x^{*}\right)\right| \leq C
$$

for some positive constant $C$ and all $n \in \mathbb{N}$. On the other hand, the scattered field corresponding to $D_{1}$ must satisfy the boundary condition and so

$$
\left|\frac{\partial w_{j}^{s}}{\partial \nu}\left(x^{*}\right)\right|=\left|\frac{\partial \Phi\left(x^{*}, x_{n}\right)}{\partial \nu}\right| \rightarrow \infty, \quad n \rightarrow \infty
$$

This contradiction shows that $D_{1}=D_{2}$

Remark 2.22. Note that with proper changes in (2.30) and in the following boundary conditions, this proof works for all boundary conditions (2.8)-(2.10). The only requirement is that

$$
\left|B \Phi\left(x^{*}, x_{n}\right)\right| \rightarrow \infty, \quad n \rightarrow \infty
$$

in order to get the contradiction at the end. In particular, it is not needed that the boundary condition imposed is the same for both obstacles $D_{1}$ and $D_{2}$. 
A similar result can also be proved for the Robin or impedance boundary condition, where the uniqueness is guaranteed not only for the obstacle, but also for the impedance function $\lambda$.

Theorem 2.23. Assume that $D_{1}$ and $D_{2}$ are two scatterers with impedances $\lambda_{1}$ and $\lambda_{2}$ such that the far-field patterns coincide for all incident plane waves with incident directions within an open non-empty subset of $\Omega_{m}$ and one fixed wave number. Then $D_{1}=D_{2}$ and $\lambda_{1}=\lambda_{2}$.

Proof. As referred in remark 2.22, with a similar proof to the one of thm 2.21 one shows that $D_{1}=D_{2}$. Let us assume that $\lambda_{1} \neq \lambda_{2}$. By Rellich's lemma we have that the total fields $u_{1}$ and $u_{2}$, corresponding to scattering by $D=D_{1}=D_{2}$ with impedances $\lambda_{1}$ and $\lambda_{2}$, respectively, coincide outside $\bar{D}$ and therefore by continuity, one finds that

$$
\frac{\partial u}{\partial \nu}+i \lambda_{1} u=\frac{\partial u}{\partial \nu}+i \lambda_{2} u=0, \text { on } \partial D
$$

where $u=u_{1}=u_{2}$. Hence $\left(\lambda_{1}-\lambda_{2}\right) u=0$. If the total field would vanish on an open set of $\partial D$, then by the boundary condition the Cauchy data of $u$ would vanish on the same set of $\partial D$ and by Holmgren's theorem and analyticity of the solution $u$, the total field $u$ would vanish in $\mathbb{R}^{m} \backslash \bar{D}$. Therefore, this cannot happen and $\lambda_{1}=\lambda_{2}$ in a $L^{2}$-sense. By the continuity of $\lambda_{1}$ and $\lambda_{2}$ we have the result.

The previous result can also be extended to the case of a point source incident field instead of plane incidence (e.g. [40]).

A uniqueness result for both sound-hard or impedance obstacle considering just a finite number of incident directions is still open, even with a priori knowledge on the size of the obstacle $D$ (analogous to thm. 2.18 for the sound-soft case). Uniqueness results for a finite number of incident directions with no a priori knowledge on the size of the obstacle for the Dirichlet case are also an open problem. However, some work as also been developed in this direction with geometrical restrictions such as the case of balls [48] and, more recently, polygonal obstacles $[1,6]$.

In chapter 5 we will be interested in recovering star-shaped obstacles, that is, with boundary of the form

$$
\Gamma=\left\{r(\hat{x}) \hat{x}: \hat{x} \in \Omega_{m}\right\}
$$

with $r \in C^{2}\left(\Omega_{m}\right)$. In this way, by a formal argument, given a complex valued function $u_{\infty}$ on $\Omega_{m}$ it makes sense to try to reconstruct one real function $r$ over $\Omega_{m}$, getting a formally overdetermined problem. In the same way, for a star-shaped domain the impedance $\lambda$ defined on the boundary can be seen as $\lambda=\lambda(\hat{x}), \hat{x} \in \Omega_{m}$. Therefore, even for the impedance case it would make sense to try to reconstruct both real functions $r$ and $\lambda$ from the knowledge of one complex valued function $u_{\infty}$ over $\Omega$. Having this formal argument in mind, we will proceed in the next chapter by suggesting a method to numerically solve the inverse scattering problem 2.15 having as data the far-field pattern $u_{\infty}$ corresponding to one single incident field. 



\section{Chapter 3}

\section{The Hybrid Method}

In this chapter, the hybrid method will be suggested as an efficient method to numerically solve the inverse problem 2.15. The denomination hybrid comes from the fact that this method can be seen as a hybrid between iterative and decomposition methods, combining ideas and gaining advantages of both of them. In the next section we will give a short introduction to these two classes of methods that have been broadly used to numerically solve the inverse obstacle scattering problem. In the following section 3.2 we will then present the general ideas of the hybrid method. A comparison between the hybrid method and its "parent" methods is presented at the end of this section. In the following sections 3.3-3.5, we will separate the presentation of the method according to each of the boundary conditions (2.8)-(2.10), since the numerical treatment must be different for each case.

\subsection{Previous Methods}

In order to present the hybrid method we will give a brief overview on methods that have previously been used to numerically solve the inverse problem under consideration, namely dividing them into three classes: iterative methods, decomposition methods and sampling methods. We will focus on regularized iterative methods and decomposition methods, since as already referred they are the "parents" of the hybrid method and so some knowledge on them is important for a better understanding of this new method. We will assume at first that the boundary condition is explicitly known a priori but we will also discuss the case of a Robin boundary condition with unknown impedance, which is equivalent to solving the problem without knowing the properties of the scatterer.

Newton's iterative methods appeared in the beginning of the 80's. These methods pose the inverse problem as an ill-posed operator equation and then solve it by regularized Newton's iterations. For instance, for a single fixed incident field $u^{i}$, the solution to the 
direct scattering problem defines the operator

$$
F: \gamma \mapsto u_{\infty}
$$

that maps the boundary $\gamma$ of some obstacle onto the far-field corresponding to scattering by that obstacle. In this sense, given the far-field pattern $u_{\infty}$, the inverse problem is equivalent to finding the solution to the nonlinear and ill-posed operator equation

$$
F(\Gamma)=u_{\infty}
$$

for the unknown boundary $\Gamma$. For sound-soft or sound-hard obstacles, regularized Newton's iterations applied to (3.2) have been studied and used for over two decades (see [21, 55, 58]). Their idea is to linearize (3.2), based on the Fréchet differentiability of the operator $F$ (see $[18,19,53 \mid)$ and iterate this procedure. More recently Kress and Rundel [40] generalized this Newton method for recovering both the unknown obstacle and the unknown impedance. Due to the ill-posedness of $F^{\prime}$, regularization is required in each iteration step. As the Fréchet derivative $F^{\prime}$ is characterized in terms of the solution to a direct problem, the main drawback of this method is that it requires a forward solver to be used at each iteration step, which is costly in terms of computations. A reasonable initial guess is also needed to start the iterations. As for the theoretical background, the convergence proofs for these methods are not yet completely satisfactory, though there has been some progress in that matter (see $[20,21,22,55]$ ).

As an alternative approach appearing on the second half of the 80's, decomposition methods take care of the ill-posedness and the nonlinearity of the inverse scattering problem separately. Their idea is the following: In a first step the total field $u$ is reconstructed from the given far-field pattern $u_{\infty}$, which is an ill-posed problem. For example, this can be done based on an analytic continuation principle (to which we will refer later on in assumption 3.2), by representing the scattered field $u^{s}$ as a layer potential over an approximate boundary $\gamma$, usually considered to be inside $D$ (see Kirsch and Kress $[30,31,32]$ ) or by approximating the fundamental solution by an Herglotz wave and insert the approximation in a single layer potential to represent the solution (see Potthast's point source method [52]). Note that in the Kirsch and Kress decomposition method, the requirement that the far-field of the potential coincides with the given far-field $u_{\infty}$ leads to an ill-posed linear integral equation that can be approximately solved via Tikhonov regularization. In the point source method an ill-posed integral equation needing regularization also arises. Then, in a second step, one tries to find the boundary $\Gamma$ as the location where the boundary condition (2.6) is satisfied in a least squares sense (or in the Dirichlet case just by plotting $|u|$ ). This second step is clearly non-linear. More recently, the point source method was adapted in [57] to reconstruct the obstacle without knowing its boundary condition. Though these methods do not need the solution to the forward problem, the reconstructions obtained are not as accurate as those obtained by Newton's iterations. As for the theoretical background, these methods are usually compared with a minimization 
problem (see[9, sec.5.4]) as we will illustrate in section 4.1, but there is a gap between the theory and the implementation of the methods.

When both these classes of methods first appeared, they required the a priori knowledge of some physical properties of the obstacle, namely the boundary condition imposed at its boundary. Therefore these methods were initially designed for sound-soft or soundhard scatterers. An alternative method for recovering scatterers with unknown boundary conditions was needed. In the second half of the 90's, a new family of methods arose the sampling or probe methods - that could deal with this problem (e.g. the linear sampling method [7], the factorization method [29], the probe algorithm [23] and the singular source method [54]). Their idea is to establish a criterion to distinguish whether a point is inside or outside $D$ based on the range of some appropriate operator, and then apply it to a grid of sampling points. Though these methods do not need a priori knowledge on the boundary condition, they usually just reconstruct the obstacle and not the boundary condition. Recently in [3], after reconstructing the obstacle by the linear sampling method, a procedure was suggested to reconstruct also the unknown impedance $\lambda$ on the boundary of this reconstructed obstacle. However a big drawback arises for this class of methods: they require a huge amount of data, namely the far-field data for many incident directions, in order to get meaningful reconstructions.

Since then several other methods were suggested to solve the inverse scattering problem (see $[4,9,10,39,56]$ for details on the state of the art), always trying to get good reconstructions with small computational cost and needing only few input data. In the next sections, the hybrid method will be suggested as a good compromise between these three aspects.

\subsection{The Hybrid Method's Basic Ideas}

The hybrid method consists of two steps in the same spirit as a decomposition method, that are iterated until some stopping criterion is achieved as in an iterative method. We will now give a general overview over the method and then particularise and give a more detailed analysis for each of the considered boundary conditions.

We will denote by $\gamma_{n}$ the current approximation to $\Gamma$ at the $n$-iteration given by the method. In the first step, the scattered field $u^{s}$ is reconstructed as a layer potential over the closed $C^{2}$-contour $\gamma_{n}$ just as in the first step of the Kirsch and Kress method [32]. In this way, let $L_{\gamma_{n}}: C\left(\gamma_{\gamma_{n}}\right) \rightarrow L^{2}\left(\mathbb{R}^{m} \backslash \gamma_{n}\right)$ be an appropriate layer potential over $\gamma_{n}$, that is, the operator $L_{\gamma_{n}}$ maps $\varphi$ living on $\gamma_{n}$ to the corresponding single-, double-or combined single-and double-layer potential. Let $L_{\gamma_{n}, \infty}$ be the corresponding far-field operator, in accordance with (2.22)-(2.24). Then, the first step consists of solving

$$
L_{\gamma_{n}, \infty} \varphi=u_{\infty}
$$


with respect to $\varphi$ and approximate the total field by

$$
u(x) \approx u^{i}(x)+\left(L_{\gamma_{n}} \varphi\right)(x), \quad x \in \mathbb{R}^{m} \backslash \gamma_{n} .
$$

Note that (3.3) is ill-posed, since $L_{\gamma_{n}, \infty}$ is a compact operator in any of the cases considered. Therefore regularization is needed. The fact that this operator is injective and has dense range is of crucial importance to assure that regularization can be used. Moreover, we also need to make use of an analytic continuation principle for $u$, since if $\gamma_{n}$ is laying inside the obstacle $D$, the equation has a solution if and only if the total field $u$ can be analytically extended up to $\gamma_{n}$. We will address this matter in assumption 3.2. In addition, if the right-hand side is perturbed with noise then in general it will lay outside the range of the operator $L_{\gamma_{n} \infty}$. Therefore, for the numerical and applicational purpose of the method, it only makes sense to search for a regularized solution. Using Tikhonov regularization, we replace (3.3) by

$$
\left(\alpha_{n} I+L_{\gamma_{n}, \infty}^{*} L_{\gamma_{n}, \infty}\right) \varphi^{(n)}=L_{\gamma_{n}, \infty}^{*} u_{\infty}
$$

with some regularization parameter $\alpha_{n}>0$ and approximate the total field $u$ by

$$
u_{n}(x)=u^{i}(x)+\left(L_{\gamma_{n}} \varphi^{(n)}\right)(x), \quad x \in \mathbb{R}^{m} \backslash \gamma_{n} .
$$

In the second step we consider the operator $G$ that for a fixed given field $u$ maps a closed $C^{2}$-contour $\gamma$ onto the trace of boundary condition of $u$ on $\gamma$, that is,

$$
G: \gamma \mapsto B_{\gamma} u
$$

where $B_{\gamma}$ is one of the boundary condition operators (2.8)-(2.10) with $\Gamma$ replaced by $\gamma$. Now, if the considered fixed field $u$ is the total field corresponding to scattering by the obstacle $D$, the solution $\Gamma$ to the inverse problem satisfies $G(\Gamma)=0$. Therefore, in the same spirit as a Newton method we linearize the previous equation based on the Fréchet differentiation of $G$ and solve

$$
G\left(\gamma_{n}\right)+G^{\prime}\left(\gamma_{n}\right) h=0
$$

in order to get a new approximation to the boundary contour given by $\gamma_{n+1}=\gamma_{n}+h$, with some abuse of notation. We look for the shift $h$ in some appropriate smooth finite dimensional space, to insure that $\gamma_{n+1}$ remains at least $C^{2}$-smooth. In practise in (3.6) we replace $u$ by the approximation (3.5). The characterization of $G^{\prime}$ depends on the boundary condition imposed. For any of the boundary conditions considered it depends on the values of $u$ and its normal derivative on $\gamma$, so in order to compute them one uses the jump relation in thm. 2.9. Now, in the spirit of a iterative method we repeat both steps until some stopping criteria is achieved. 
Remark 3.1. In the initial Kirsch and Kress method [32], no iteration on the curve $\gamma$ was made, since it was not seen as an approximation to $\Gamma$. In fact, the curve $\gamma$ had a role of an auxiliary curve to recover the total field $u$. Then one would obtain a linearized equation similar to (3.7) that was solved iteratively using always the fixed field obtained in the first step. In this way it was of crucial importance that the approximation (3.5) was defined over $\Gamma$ and so one needed the assumption that $\gamma$ is inside $D$. The way the hybrid method was constructed eliminates this need, since both $u$ and its derivatives are evaluated at each step only over the current approximation $\gamma_{n}$.

The hybrid method is closer to a decomposition method than to a Newton method. It does not need a forward solver and also separates the ill-posedness from the non-linearity of the problem into two steps in each iteration. The previous remark clarifies the changes made to a particular decomposition method - the Kirsch and Kress method [32] - in order to allow it to be iterated, reviving it with a more competitive version. Moreover, the hybrid method is more effective than the Kirsch and Kress method in the sense that it gets better reconstructions (as we will see in section 5), being able to compete with approximations obtained by the usual regularized Newton method. In this way, this method provides a good compromise between required data, computational costs and numerical accuracy.

In the next three sections we will proceed by characterizing $G^{\prime}$ for each of the considered boundary conditions. At the end of section 3.5 we will compare the obtained characterization of $G^{\prime}$ with the Fréchet derivative of the far-field operator $F$ in (3.1) that gives rise to an iterative Newton method as mentioned previously, relating in this way the hybrid method to this other iterative method.

\subsection{The Hybrid Method for the Dirichlet Case}

In this section we will consider the hybrid method for the numerical solution to the inverse obstacle scattering problem 2.15 with Dirichlet boundary condition. This means the solution $u^{s}$ to the direct problem satisfies

$$
\begin{array}{r}
\Delta u^{s}+k^{2} u^{s}=0, \quad x \in \mathbb{R}^{m} \backslash \bar{D}, \\
u=0, \quad x \in \Gamma, \\
\lim _{r \rightarrow \infty} r^{\frac{m-1}{2}}\left(\frac{\partial u^{s}}{\partial r}-i k u^{s}\right)=0,
\end{array}
$$

where again the total field $u$ is the sum of the given incident field $u^{i}$ and the scattered field $u^{s}$. The goal is to recover the domain $D$ from the knowledge of the far-field pattern $u_{\infty}$ for one incident plane wave $u^{i}(x)=e^{i k x \cdot d},|d|=1$. 
We will consider that the solution to the inverse problem can be parameterized, that is,

$$
\Gamma=\left\{z_{*}(t): t \in X\right\}
$$

where the parameterization $z_{*} \in C^{2}(X)$ is such that $\Gamma$ is $C^{2}$-smooth (in $\mathbb{R}^{2}$ we will assume that $z_{*} \in C^{2}([0,2 \pi])$ is $2 \pi$-periodic and is counter-clockwise oriented) and has the following mapping properties

$$
\left\{\begin{array}{lll}
z: X \rightarrow \mathbb{R}^{2}, & X:=[0,2 \pi], & m=2, \\
z: X \rightarrow \mathbb{R}^{3}, & X:=[0, \pi] \times[0,2 \pi], & m=3 .
\end{array}\right.
$$

At the $n$-iteration of the hybrid method we will consider that the current approximation $\gamma_{n}$ to the correct boundary $\Gamma$ is given by

$$
\gamma_{n}=\left\{z_{n}(t): t \in X\right\}
$$

Later on we will assume that $z_{n} \in U$, where $U$ is a finite dimensional subspace of the previous parameterization space, that is

$$
U \subset\left\{z: z \in C^{2}(X), z \text { is } X \text {-periodic }\right\}
$$

Following the comments on the solvability of (3.3), we will now state an assumption that will be of crucial importance throughout this work.

Assumption 3.2 (Analytic Continuation Principle). The solution $u^{s}$ to the direct problem of scattering by $D$ can be analytically extended as a solution to the Helmholtz equation in a neighbourhood of the boundary $\Gamma$ of $D$.

Remark 3.3. If the boundary $\Gamma$ is analytic, then assumption 3.2 holds (see [13]).

Assuming that $\gamma_{n}$ is sufficiently close to $\Gamma$ in a way that the solution $u^{s}$ to the direct problem of scattering by $D$ can be analytically extended up to $\gamma_{n}$ and assuming that $k^{2}$ is not an interior Dirichlet eigenvalue of the negative Laplacian for the interior of $\gamma_{n}$, we can represent the scattered field $u^{s}$ as a single layer potential over $\gamma_{n}$ (see $[8$, thm. 3.30]), that is

$$
u^{s}(x)=\int_{\gamma_{n}} \Phi(x, y) \varphi(y) d s(y), \quad x \in \mathbb{R}^{m} \backslash \gamma_{n}
$$

with density $\varphi \in C\left(\gamma_{n}\right)$.

Remark 3.4. We choose a single-layer representation because it leads to less complexity later on in the implementation. However, the condition on the wave number $k$ is needed and can not be guaranteed a priori for the successive approximations $\gamma_{n}$. We also note that for the Neumann and Robin cases we will use a combined single-and double-layer potential in order to illustrate the differences in the implementation. 
Due to analyticity of the single layer potential in $\mathbb{R}^{m} \backslash \gamma_{n}$, it is clear that if the analytic continuation principle 3.2 does not hold, then the previous representation would not make sense for the cases where $\gamma_{n} \cap D \neq \emptyset$. One knows that the exterior trace to the boundary of the previous potential is given by

$$
u^{s}(x)=\left(S_{\gamma_{n}} \varphi\right)(x), \quad x \in \gamma_{n}
$$

where $S_{\gamma_{n}}: C\left(\gamma_{n}\right) \rightarrow C\left(\gamma_{n}\right)$ is the single-layer operator (2.18). By the asymptotics of the single layer potential the far-field must satisfy the equation

$$
S_{\gamma_{n}, \infty} \varphi=u_{\infty} \text { in } \Omega_{m}
$$

with the far-field operator $S_{\gamma, \infty}: C(\gamma) \rightarrow C\left(\Omega_{m}\right)$ given by

$$
S_{\gamma, \infty} \varphi=\varrho_{m} \int_{\gamma} e^{-i k \hat{x} \cdot y} \varphi(y) d s(y), \quad \hat{x} \in \Omega_{m},
$$

with $\varrho_{m}$ given as in (2.14). As referred in the previous section, the previous operator is compact (since it has a continuous kernel) so (3.8) must be replaced by a regularized equation. In order to show that a regularization scheme is applicable one needs to show that the operator $S_{\gamma_{n}, \infty}$ is injective.

Theorem 3.5. Assume that $k^{2}$ is not an interior Dirichlet eigenvalue of the negative Laplacian with respect to the open bounded domain $D_{\gamma}$ with boundary $\gamma$. Then the operator $S_{\gamma, \infty}: L^{2}(\gamma) \rightarrow L^{2}\left(\Omega_{m}\right)$ is injective and has dense range.

Proof. Assume that $\psi \in L^{2}(\gamma)$ satisfies $S_{\gamma, \infty} \psi=0$. Then the single-layer potential

$$
v(x)=\int_{\gamma} \Phi(x, y) \psi(y) d s(y), \quad x \in \mathbb{R}^{m} \backslash \gamma
$$

has a vanishing far-field. By Rellich's lemma and analyticity we conclude that $v$ vanishes in $\mathbb{R}^{m} \backslash \overline{D_{\gamma}}$. By continuity up to the boundary we conclude that $S_{\gamma} \psi=0$ over $\gamma$. As $k^{2}$ is not an interior Dirichlet eigenvalue with respect to the open bounded domain $D_{\gamma}$ we know that $S_{\gamma}$ is injective (see [8, thm.3.30]) and so we conclude that $\psi=0$, proving injectivity of $S_{\gamma}$.

To prove denseness one shows by a similar argument that the adjoint operator $S_{\gamma}^{*}$ is injective and concludes that $S_{\gamma}$ is therefore dense, since for a linear bounded operator $A$ the closure of the range of $A$ is the orthogonal complement of the nullspace of $A^{*}$ (for details see $[9$, thm.5.17]).

Using Tikhonov regularization, as referred in the previous section, we need to replace (3.8) by

$$
\left(\alpha_{n} I+S_{\gamma_{n}, \infty}^{*} S_{\gamma_{n}, \infty}\right) \varphi^{(n)}=S_{\gamma_{n}, \infty}^{*} u_{\infty}
$$


solving it with respect to $\varphi^{(n)}$ for some regularization parameter $\alpha_{n}>0$ decreasing with $n$. The scattered field $u^{s}$ can now be approximated by

$$
u_{n}^{s}(x)=\int_{\gamma_{n}} \Phi(x, y) \varphi^{(n)} d s(y), \quad x \in \mathbb{R}^{m} \backslash \gamma_{n} .
$$

and using the jump relations we also get the approximations to $u^{s}$ and its exterior normal derivative $\partial u^{s} / \partial \nu$ on $\gamma_{n}$ given by

$$
\begin{aligned}
u_{n}^{s}(x) & =\left(S_{\gamma_{n}} \varphi^{(n)}\right)(x), \quad x \in \gamma_{n}, \\
\frac{\partial u_{n}^{s}}{\partial \nu}(x) & =-\frac{1}{2} \varphi^{(n)}+\left(K_{\gamma_{n}}^{*} \varphi^{(n)}\right)(x), \quad x \in \gamma_{n},
\end{aligned}
$$

respectively.

For a fixed analytic field $u$, we now define the operator $G_{D}$ that maps the parameterization $z$ of the contour $\gamma$ to the exterior trace of the Dirichlet boundary condition of that field $u$ over $\gamma$, that is,

$$
G_{D}: z \mapsto u \circ z .
$$

If the field $u$ is the total field, then in order to find the position of the boundary of the obstacle $D$ as the location where the boundary condition is satisfied, we want to find the solution to

$$
G_{D}(z)=0 .
$$

In the spirit of a Newton method we now linearize the previous equation around $z_{n}$ and solve the linearized equation

$$
G_{D}\left(z_{n}\right)+G_{D}^{\prime}\left(z_{n}\right) h=0 \quad \text { in } X
$$

with respect to the shift $h$. In the next theorem we characterize the Fréchet derivative of $G_{D}$.

Theorem 3.6. The operator $G_{D}: C^{2}(X) \rightarrow C(X)$ is Fréchet differentiable and the Fréchet derivative is given by

$$
G_{D}^{\prime}(z) h=(\operatorname{grad} u \circ z) \cdot h .
$$

Proof. By the Taylor formula, the Fréchet differentiability of $G_{D}$ is a direct consequence of the analyticity of $u$ and the $C^{2}$-smoothness of $z$. Moreover, from the Taylor formula for $u$ one gets for each $t \in X$ that

$$
u(z(t)+h(t))=u(z(t))+\operatorname{grad} u(z(t)) \cdot h(t)+O\left(|h(t)|^{2}\right),
$$

as $\|h\|_{\infty} \rightarrow 0$. Therefore by definition of $G_{D}$ we have

$$
\left\|G_{D}(z+h)-G_{D}(z)-(\operatorname{grad} u \circ z) \cdot h\right\|_{\infty}=O\left(\|h\|_{\infty}^{2}\right)
$$

as $\|h\|_{\infty} \rightarrow 0$ and by definition of the Fréchet derivative one has the result. 
With this characterization, equation (3.13) can be rewritten in the following way

$$
\left(\operatorname{grad} u \circ z_{n}\right) \cdot h=-u \circ z_{n} \text { in } X \text {. }
$$

In this way, at each iteration $n$ we approximate $u^{s}$ by $u_{n}^{s}$ obtained in the first step of the iteration and solve

$$
\left(\left(\operatorname{grad} u_{n}^{s}+\operatorname{grad} u^{i}\right) \circ z_{n}\right) \cdot h=-\left(u_{n}^{s}+u^{i}\right) \circ z_{n} \quad \text { in } X
$$

with respect to $h$ in a least squares sense, obtaining the new approximation $\gamma_{n+1}$ parameterized by $z_{n+1}=z_{n}+h$. Note that we use the jump relations (3.11) and (3.12) to compute the terms involved, through the decomposition

$$
\left.\operatorname{grad} u\right|_{\gamma_{n}}=\left.\nu \frac{\partial u}{\partial \nu}\right|_{\gamma_{n}}+\nabla_{t} u
$$

where $\nabla_{t} u$ represents the surface gradient of $u$, which in $\mathbb{R}^{2}$ reduces itself to the tangential derivative times the tangential unit vector. We now repeat the two steps until some stopping criteria is fulfilled. The details on the numerical implementation will be given in sections 5.1.2 and 5.2.2 for $\mathbb{R}^{2}$ and $\mathbb{R}^{3}$, respectively.

Remark 3.7. This method can also be extended to recover sound-soft cracks. The main difference is that one needs to choose the right or left hand side normal derivative in (3.15) in an appropriate way. A further penalty term on the length of the crack is also needed to prevent it from degenerating to a point. For details we refer to [42].

Remark 3.8. Note that to show solvability of (3.13) we would need to show that $G_{D}^{\prime}\left(z_{n}\right)$ is injective. However this can only be done if we are over the correct boundary $\Gamma$, if $u$ is considered to be the exact total field and if there exists an open set of $\Gamma$ where $h \cdot \nu \neq 0$. In this case, by the boundary condition we have that if

$$
0=G_{D}^{\prime}\left(z_{*}\right) h=h \cdot \operatorname{grad} u \circ z_{*}=h \cdot \nu \frac{\partial u}{\partial \nu}
$$

then $h=0$ everywhere. By contradiction, assume than this is not the case. Then, as there exists an open subset of $\Gamma$ where $h \cdot \nu \neq 0$, the normal derivative of $u$ would need to vanish on that open subset of $\Gamma$. By the boundary condition and Holmgren's theorem we conclude that the total field $u=0$ in $\mathbb{R}^{m} \backslash \bar{D}$, which can not happen since the scattered field goes to zero at infinity and the incident field does not. A similar result can be shown for the Neumann and Robin boundary condition (see [59, thm.5]). 


\subsection{The Hybrid Method for the Neumann Case}

We will now adapt the previous approach to solve the inverse problem 2.15 with Neumann boundary condition. In this way, the solution $u^{s}$ to the direct problem satisfies

$$
\begin{array}{r}
\Delta u^{s}+k^{2} u^{s}=0, \quad x \in \mathbb{R}^{m} \backslash \bar{D}, \\
\frac{\partial u}{\partial \nu}=0, \quad x \in \Gamma, \\
\lim _{r \rightarrow \infty} r^{\frac{m-1}{2}}\left(\frac{\partial u^{s}}{\partial r}-i k u^{s}\right)=0,
\end{array}
$$

where again the total field $u$ is the sum of the given incident field $u^{i}$ and the scattered field $u^{s}$. Again, the goal is to recover the domain $D$ from the knowledge of the far-field pattern $u_{\infty}$ for one incident plane wave $u^{i}(x)=e^{i k x \cdot d},|d|=1$.

As already mentioned, the analytic continuation principle is of major importance for reconstructing the total field using the Kirsch and Kress idea. Again we assume that the solution $u^{s}$ to the direct scattering problem for $D$ can be analytically extended up to $\gamma_{n}$. In order to loose the assumption on $k^{2}$ not being an interior eigenvalue, we will now represent the scattered field $u^{s}$ as a combined single-and double-layer potential over $\gamma_{n}$ (see [8, thm.3.34]). However, this combined layer representation is of course harder to implement numerically. In this way we have

$$
u^{s}(x)=\int_{\gamma_{n}}\left(\frac{\partial \Phi(x, y)}{\partial \nu(y)}-i \eta \Phi(x, y)\right) \varphi(y) d s(y), \quad x \in \mathbb{R}^{m} \backslash \gamma_{n}
$$

for some coupling term $\eta>0$ and density $\varphi \in C^{1, \alpha}\left(\gamma_{n}\right)$. For the combined layer approach we need more regularity assumptions on the density $\varphi$ since later on we will need to use the mapping properties of the operator $T$ defined in (2.21) to have, in particular, that $(T \varphi) \in C\left(\gamma_{n}\right)$. This will then ensure that the normal trace of $u^{s}$ is continuous over $\gamma_{n}$.

Remark 3.9. As in [9], such a strong regularity assumption on $\varphi$ is not needed if we use the smoothing operator $S_{0}$ in our ansatz for $u^{s}$, where $S_{0}$ is the single-layer operator with kernel $\Phi$ being the fundamental solution to the Laplace equation. Then we could represent $u^{s}$ as

$$
u^{s}(x)=\int_{\gamma_{n}} \frac{\partial \Phi(x, y)}{\partial \nu(y)}\left(S_{0}^{2} \varphi\right)(y)-i \eta \Phi(x, y) \varphi(y) d s(y), \quad x \in \mathbb{R}^{m} \backslash \gamma_{n}
$$

with $\varphi \in C\left(\gamma_{n}\right)$, since $S_{0}^{2}: C\left(\gamma_{n}\right) \rightarrow C^{1, \alpha}\left(\gamma_{n}\right)$ and therefore $\left(T S_{0}^{2} \varphi\right) \in C(\gamma)$. Though this ansatz solves the problem theoretically, we do not take it for our analysis because for a computational purpose this operator is much more complicated and its numerical treatment is more costly. 
By the jump relations one gets that the exterior trace of $u^{s}$ over $\gamma_{n}$ is given by

$$
u^{s}=\frac{\varphi}{2}+\left(K_{\gamma_{n}}-i \eta S_{\gamma_{n}}\right) \varphi \quad \text { on } \gamma_{n}
$$

where $S_{\gamma_{n}}: C^{1, \alpha}\left(\gamma_{n}\right) \rightarrow C\left(\gamma_{n}\right)$ is the single-layer operator previously defined in (2.18) and $K_{\gamma_{n}}: C^{1, \alpha}\left(\gamma_{n}\right) \rightarrow C\left(\gamma_{n}\right)$ is the double-layer operator (2.19). The normal trace of $u^{s}$ has also a jump and is given by

$$
\frac{\partial u^{s}}{\partial \nu}=i \eta \frac{\varphi}{2}+\left(T_{\gamma_{n}}-i \eta K_{\gamma_{n}}^{*}\right) \varphi \quad \text { on } \gamma_{n}
$$

where $K_{\gamma_{n}}^{*}: C^{1, \alpha}\left(\gamma_{n}\right) \rightarrow C\left(\gamma_{n}\right)$ is the normal derivative of the single-layer as defined in (2.20) and $T_{\gamma_{n}}: C^{1, \alpha}\left(\gamma_{n}\right) \rightarrow C\left(\gamma_{n}\right)$ is the normal derivative of the double-layer as defined in (2.21). Note that by the compact imbedding of $C^{0, \alpha}(\gamma)$ in $C(\gamma)$ and by the mapping properties of $S, K, K^{*}$ and $T$ given in thm. 2.10 all these four operators are compact.

By the asymptotics of the single-and double-layer potential the far-field must satisfy the equation

$$
F_{\gamma_{n}, \infty} \varphi=u_{\infty} \quad \text { on } \Omega_{m}
$$

with $F_{\gamma, \infty}:=\left(K_{\gamma, \infty}-i \eta S_{\gamma, \infty}\right)$, where the operators $S_{\gamma, \infty}, K_{\gamma, \infty}: C^{1, \alpha}\left(\gamma_{n}\right) \rightarrow C\left(\gamma_{n}\right)$ are defined in (2.22) and (2.23). Again the operator $F_{\gamma, \infty}$ is compact so a regularized solution must be searched. As one needs an Hilbert space setting, we will consider the operator $F_{\gamma, \infty}: H^{m}(\gamma) \rightarrow L^{2}(\gamma)$, since by the Sobolev imbedding theorems one has that $H^{m}(\gamma) \subset C^{1, \alpha}(\gamma)$ for $\gamma$ a closed hyper-surface in $\mathbb{R}^{m}$.

Theorem 3.10. The operator $F_{\gamma, \infty}: H^{m}(\gamma) \rightarrow L^{2}\left(\Omega_{m}\right)$ is injective and has dense range.

Proof. The proof goes in a similar way as the proof of theorem 3.5. For details, we refer to [9] for the injectivity proof and to [26] for the denseness result.

Let us suppose that $F_{\gamma, \infty} \psi=0$. Then, by definition of $F_{\gamma, \infty}$, the far-field of

$$
v=\int_{\gamma}\left(\frac{\partial \Phi(x, y)}{\partial \nu(y)}-i \eta \Phi(x, y)\right) \psi(y) d s(y), \quad x \in \mathbb{R}^{m} \backslash \gamma
$$

vanishes. Therefore by Rellich's lemma, $v=0$ in $\mathbb{R}^{m} \backslash D_{\gamma}$, where $D_{\gamma}$ is the bounded domain with boundary $\gamma$. As $v$ is a solution to the direct scattering problem it is continuous up to the boundary and therefore one gets

$$
\left(I / 2+K_{\gamma}-i \eta S_{\gamma}\right) \psi=0
$$

and by injectivity of this operator that $\psi=0$. As for the denseness result, we have that the boundary data to far-field operator $G:=F_{\gamma, \infty} L_{\gamma}^{-1}: H^{m}(\gamma) \rightarrow L^{2}\left(\Omega_{m}\right)$ has dense range (see cor.3.2 in [26]) and so $F_{\gamma, \infty}$ must have dense range. 
Using Tikhonov regularization we then solve

$$
\left(\alpha_{n} I+F_{\gamma_{n}, \infty}^{*} F_{\gamma_{n}, \infty}\right) \varphi^{(n)}=F_{\gamma_{n}, \infty}^{*} u_{\infty}
$$

with respect to $\varphi^{(n)}$ for some regularization parameter $\alpha_{n}>0$ decreasing with the iteration $n$. In a similar way as for the Dirichlet case we find an approximation

$$
u_{n}^{s}(x)=\int_{\gamma_{n}}\left(\frac{\partial \Phi(x, y)}{\partial \nu(y)}-i \eta \Phi(x, y)\right) \varphi^{(n)}(y) d s(y), \quad x \in \mathbb{R}^{m} \backslash \gamma_{n}
$$

to the scattered field $u^{s}$. In the same way, by the jump relations we have approximations to the exterior trace and to the normal trace of $u$ given by

$$
\begin{aligned}
u_{n}^{s} & =\frac{\varphi^{(n)}}{2}+\left(K_{\gamma_{n}}-i \eta S_{\gamma_{n}}\right) \varphi^{(n)} \quad \text { on } \gamma_{n}, \\
\frac{\partial u_{n}^{s}}{\partial \nu} & =i \eta \frac{\varphi^{(n)}}{2}+\left(T_{\gamma_{n}}-i \eta K_{\gamma_{n}}^{*}\right) \varphi^{(n)} \quad \text { on } \gamma_{n} .
\end{aligned}
$$

Following the same procedure as for the Dirichlet case, we now define the operator $G_{N}$ that maps the parameterization $z$ of the contour $\gamma$ to the exterior trace of the Neumann boundary condition of some fixed analytic field $u$ over $\gamma$, that is,

$$
G_{N}: z \mapsto(\nu \cdot \operatorname{grad} u) \circ z .
$$

Again if $u$ is the total field, we want to find the solution to

$$
G_{N}(z)=0
$$

in order to find the position of the boundary of the obstacle $D$ as the location where the boundary condition is satisfied. In the same way, based on the Fréchet differentiability of $G_{N}$, we linearize the previous equation around $z_{n}$ and solve the linearized equation

$$
G_{N}\left(z_{n}\right)+G_{N}^{\prime}\left(z_{n}\right) h=0 \quad \text { in } X
$$

with respect to the shift $h$, using $u_{n}$ as approximation to the total field $u$. A characterization for the Fréchet derivative of $G_{N}$ is now needed. We will split the analysis for the two-dimensional $(m=2)$ and three-dimensional $(m=3)$ case.

\section{Two-dimensional Case}

For simplicity we start by stating this characterization for $m=2$, that is, in $\mathbb{R}^{2}$. We note that in this case $z:[0,2 \pi] \rightarrow \mathbb{R}^{2}$ is oriented in the counter-clockwise direction and that in an equivalent way we can write

$$
G_{N}: z \mapsto \frac{z^{\perp}}{\left|z^{\prime}\right|} \cdot \operatorname{grad} u \circ z .
$$


where $z^{\perp}=\left(z_{2},-z_{1}\right)$. Note that $G_{N}$ really maps the parameterization $z$ to the Neumann boundary condition, since for a counter-clockwise parameterization $z$ the exterior normal vector is given by

$$
\nu \circ z=\frac{z^{\prime \perp}}{\left|z^{\prime}\right|} .
$$

We prepare the theorem for this characterization by introducing the notation $\tau$ for the unit tangential vector to the boundary in the counter-clockwise direction.

Theorem 3.11. The operator $G_{N}: C^{2}[0,2 \pi] \rightarrow C[0,2 \pi]$ is Fréchet differentiable and its derivative is given by

$$
G_{N}^{\prime}(z) h=-\frac{\left(h^{\prime} \cdot \nu\right)}{\left|z^{\prime}\right|} \frac{\partial u}{\partial \tau} \circ z+(h \cdot \tau)\left[\frac{\partial^{2} u}{\partial \tau \partial \nu} \circ z-H \frac{\partial u}{\partial \tau} \circ z\right]+(h \cdot \nu) \frac{\partial^{2} u}{\partial \nu^{2}} \circ z
$$

in $[0,2 \pi]$, where $H$ stands for the curvature.

Proof. Again, the Fréchet differentiability of $G_{N}$ is a consequence of the analyticity of $u$ and the $C^{2}$-smoothness of $z$.

Let $h$ be sufficiently small to ensure that

$$
\gamma_{z+h}=\{z(s)+h(s): s \in[0,2 \pi]\}
$$

describes a closed curve.

We decompose

$$
\begin{aligned}
G_{N}(z+h)-G_{N}(z)= & \left(\frac{z^{\prime \perp}+h^{\prime \perp}}{\left|z^{\prime}+h^{\prime}\right|}-\frac{z^{\prime \perp}}{\left|z^{\prime}\right|}\right) \cdot(\operatorname{grad} u \circ(z+h))+ \\
& +\frac{z^{\prime \perp}}{\left|z^{\prime}\right|} \cdot(\operatorname{grad} u \circ(z+h)-\operatorname{grad} u \circ z)
\end{aligned}
$$

and treat both terms on the right hand side separately. Using Taylor's formula, we begin by noting that

$$
\begin{aligned}
\frac{z^{\prime \perp}+h^{\prime \perp}}{\left|z^{\prime}+h^{\prime}\right|}-\frac{z^{\prime \perp}}{\left|z^{\prime}\right|} & =\frac{h^{\prime \perp}}{\left|z^{\prime}\right|}-\frac{z^{\prime \perp}\left(z^{\prime} \cdot h^{\prime}\right)}{\left|z^{\prime}\right|^{3}}+O\left(\left|h^{\prime}\right|^{2}\right) \\
& =\frac{1}{\left|z^{\prime}\right|}\left(h^{\prime \perp}-\left(h^{\prime} \cdot \tau\right) \nu\right)+O\left(\left|h^{\prime}\right|^{2}\right)
\end{aligned}
$$

since $\tau=z^{\prime} /\left|z^{\prime}\right|$. Using

$$
\operatorname{grad} u \circ(z+h)-\operatorname{grad} u \circ z=O(|h|)
$$


consequently we have

$$
\begin{aligned}
& \left(\frac{z^{\perp}+h^{\prime \perp}}{\left|z^{\prime}+h^{\prime}\right|}-\frac{z^{\prime \perp}}{\left|z^{\prime}\right|}\right) \cdot(\operatorname{grad} u \circ(z+h))= \\
& \quad=\frac{1}{\left|z^{\prime}\right|}\left(h^{\prime \perp}-\left(h^{\prime} \cdot \tau\right) \nu\right) \cdot \operatorname{grad} u \circ z+O\left(\left|h^{\prime}\right|^{2}\right)+O\left(\left|h^{\prime}\right||h|\right) .
\end{aligned}
$$

We now perform a change of variables in a neighbourhood of $\gamma$ by

$$
x(s, \varepsilon)=z(s)+\varepsilon \nu(s), \quad s \in[0,2 \pi], \varepsilon \in\left(-\varepsilon_{0}, \varepsilon_{0}\right)
$$

and set

$$
v(s, \varepsilon)=u(z(s)+\varepsilon \nu(s)) .
$$

In the new coordinate system we have that (e.g [62])

$$
\operatorname{grad} v(s, \varepsilon)=\frac{1}{\left|z^{\prime}(s)+\varepsilon \nu^{\prime}(s)\right|^{2}} \frac{\partial v}{\partial s}(s, \varepsilon)\left[z^{\prime}(s)+\varepsilon \nu^{\prime}(s)\right]+\frac{\partial v}{\partial \varepsilon}(s, \varepsilon) \nu(s) .
$$

Therefore we can rewrite (3.23) as

$$
\left(\frac{z^{\prime \perp}+h^{\prime \perp}}{\left|z^{\prime}+h^{\prime}\right|}-\frac{z^{\prime \perp}}{\left|z^{\prime}\right|}\right) \cdot(\operatorname{grad} u \circ(z+h))=-\frac{\left(h^{\prime} \cdot \nu\right)}{\left|z^{\prime}\right|^{2}} \frac{\partial v}{\partial s}+O\left(\left|h^{\prime}\right|^{2}\right)+O\left(\left|h^{\prime}\right||h|\right)
$$

using the equalities

$$
h^{\prime \perp} \cdot \tau=-h^{\prime} \cdot \nu \quad \text { and } \quad h^{\prime \perp} \cdot \nu=h^{\prime} \cdot \tau .
$$

We now consider the second term on the right hand side of (3.22). Taylor's formula and the relations $\nu \cdot z^{\prime}=0$ and $\nu^{\prime} \cdot \nu=0$ imply that

$$
\begin{aligned}
\nu(s) \cdot & {[\operatorname{grad} v(s+\sigma, \epsilon)-\operatorname{grad} v(s, 0)]=} \\
= & {\left[\frac{\partial^{2} v}{\partial s \partial \varepsilon}(s, 0)-H(s) \frac{\partial v}{\partial s}(s, 0)\right] \sigma+\frac{\partial^{2} v}{\partial \varepsilon^{2}}(s, 0) \epsilon+O\left(\sigma^{2}+\epsilon^{2}\right) }
\end{aligned}
$$

where the curvature $H$ in two dimensions is given by

$$
H=\frac{z_{1}^{\prime} z_{2}^{\prime \prime}-z_{2}^{\prime} z_{1}^{\prime \prime}}{\left|z^{\prime}\right|^{3}}=-\frac{z^{\prime \prime} \cdot \nu}{\left|z^{\prime}\right|^{2}} .
$$

In view of the second term on the right hand side of (3.22) we want to choose the pair $(\sigma, \epsilon)$ such that

$$
z(s)+h(s)=z(s+\sigma)+\epsilon \nu(s+\sigma) .
$$


By Taylor's formula, we note that

$$
h(s)-\epsilon \nu(s)+O(\sigma \epsilon)=z(s+\sigma)-z(s)=z^{\prime}(s) \sigma+O\left(\sigma^{2}\right)
$$

and therefore

$$
h(s)=z^{\prime}(s) \sigma+\epsilon \nu(s)+O(\sigma \epsilon)+O\left(\sigma^{2}\right) .
$$

Comparing the previous expression with the decomposition

$$
h(s)=\frac{(h(s) \cdot \tau(s))}{\left|z^{\prime}(s)\right|} z^{\prime}(s)+(h(s) \cdot \nu(s)) \nu(s),
$$

we have

$$
\sigma=\frac{h \cdot \tau}{\left|z^{\prime}\right|} \quad \text { and } \quad \epsilon=h \cdot \nu
$$

Therefore, we can write the second term on the right hand side of (3.22) as

$$
\begin{aligned}
\nu(s) \cdot & (\operatorname{grad} u(z(s)+h(s))-\operatorname{grad} u(z(s)))= \\
= & {\left[\frac{\partial^{2} v}{\partial s \partial \varepsilon}(s, 0)-H(s) \frac{\partial v}{\partial s}(s, 0)\right] \frac{(h(s) \cdot \tau(s))}{\left|z^{\prime}(s)\right|} } \\
& +\frac{\partial^{2} v}{\partial \varepsilon^{2}}(s, 0)(h(s) \cdot \nu(s))+O\left(|h|^{2}\right) .
\end{aligned}
$$

Inserting (3.25) and (3.27) into (3.22) and by definition of the Fréchet derivative

$$
\left|G_{N}(z+h)-G_{N}(z)-G_{N}^{\prime}(z) h\right|=O\left(\|h\|_{C^{2}}^{2}\right), \quad\|h\|_{C^{2}} \rightarrow 0,
$$

one gets

$$
\begin{aligned}
G_{N}^{\prime}(z(s)) h(s)= & -\frac{\left(h^{\prime}(s) \cdot \nu(s)\right)}{\left|z^{\prime}\right|^{2}} \frac{\partial v}{\partial s}(s, 0)+\frac{\partial^{2} v}{\partial \varepsilon^{2}}(s, 0)(h(s) \cdot \nu(s)) \\
& +\left[\frac{\partial^{2} v}{\partial s \partial \varepsilon}(s, 0)-H(s) \frac{\partial v}{\partial s}(s, 0)\right] \frac{(h(s) \cdot \tau(s))}{\left|z^{\prime}(s)\right|}
\end{aligned}
$$

and by the relations

$$
\frac{\partial v}{\partial \varepsilon}(s, 0)=\frac{\partial u}{\partial \nu}(z(s))
$$

and

$$
\frac{1}{\left|z^{\prime}(s)\right|} \frac{\partial v}{\partial s}(s, 0)=\frac{\partial u}{\partial \tau}(z(s))
$$

the result follows. 
In practice one wants to avoid computing the term $\partial^{2} u / \partial^{2} \nu$ appearing in (3.21). Therefore, in the following corollary this term is eliminated by using the fact that $u$ satisfies the Helmholtz equation.

Corollary 3.12. Provided that the field $u$ satisfies the Helmholtz equation, the Fréchet derivative of $G_{N}: C^{2}[0,2 \pi] \rightarrow C[0,2 \pi]$ is given by

$$
\begin{aligned}
G_{N}^{\prime}(z) h= & -k^{2}(h \cdot \nu) u \circ z-\frac{\partial}{\partial \tau}\left(h \cdot \nu\left(\frac{\partial u}{\partial \tau} \circ z\right)\right) \\
& +H(h \cdot \nu) \frac{\partial u}{\partial \nu} \circ z+(h \cdot \tau) \frac{\partial^{2} u}{\partial \tau \partial \nu} \circ z
\end{aligned}
$$

in $[0,2 \pi]$, where again $H$ holds for the curvature.

Proof. Using the same change of variables (3.24) as in the previous proof, for the Laplace operator we have that

$$
\begin{aligned}
\Delta v(s, \varepsilon)= & \frac{1}{\left|z^{\prime}(s)+\varepsilon \nu^{\prime}(s)\right|}\left\{\frac{\partial}{\partial s}\left(\frac{1}{\left|z^{\prime}(s)+\varepsilon \nu^{\prime}(s)\right|} \frac{\partial v}{\partial s}(s, \varepsilon)\right)\right. \\
& \left.+\frac{\partial}{\partial \varepsilon}\left(\left|z^{\prime}(s)+\varepsilon \nu^{\prime}(s)\right| \frac{\partial v}{\partial \varepsilon}(s, \varepsilon)\right)\right\} .
\end{aligned}
$$

Therefore we can write

$$
\begin{aligned}
\frac{\partial^{2} v}{\partial \varepsilon^{2}}(s, 0)= & -k^{2} v(s, 0)+\frac{z^{\prime}(s) \cdot z^{\prime \prime}(s)}{\left|z^{\prime}(s)\right|^{4}} \frac{\partial v}{\partial s}(s, 0) \\
& -\frac{1}{\left|z^{\prime}(s)\right|^{2}} \frac{\partial^{2} v}{\partial s^{2}}(s, 0)+H(s) \frac{\partial v}{\partial \varepsilon}(s, 0)
\end{aligned}
$$

since $u$ satisfies the Helmholtz equation. This comes from the fact that in the new coordinate system (e.g. [62])

$$
\begin{aligned}
\Delta v(s, \epsilon) \rightarrow & -\frac{z^{\prime}(s) \cdot z^{\prime \prime}(s)}{\left|z^{\prime}(s)\right|^{4}} \frac{\partial v}{\partial s}(s, 0)+\frac{1}{\left|z^{\prime}(s)\right|^{2}} \frac{\partial^{2} v}{\partial s^{2}}(s, 0) \\
& +\frac{z^{\prime}(s) \cdot \nu^{\prime}(s)}{\left|z^{\prime}(s)\right|^{2}} \frac{\partial v}{\partial \varepsilon}(s, 0)+\frac{\partial^{2} v}{\partial \varepsilon^{2}}(s, 0),
\end{aligned}
$$

as $\epsilon \rightarrow 0$ and from the identity

$$
\nu^{\prime} \cdot z^{\prime}=z^{\prime \prime} \cdot \nu=-\left|z^{\prime}\right|^{2} H .
$$


Substituting (3.32) in (3.28) one gets the characterization

$$
\begin{aligned}
G_{N}^{\prime}(z) h= & -\frac{\left(h^{\prime} \cdot \nu\right)}{\left|z^{\prime}\right|^{2}} \frac{\partial v}{\partial s}+\left[\frac{\partial^{2} v}{\partial s \partial \varepsilon}-H \frac{\partial v}{\partial s}\right] \frac{(h \cdot \tau)}{\left|z^{\prime}\right|} \\
& +\left[-k^{2} v+\frac{z^{\prime} \cdot z^{\prime \prime}}{\left|z^{\prime}\right|^{4}} \frac{\partial v}{\partial s}-\frac{1}{\left|z^{\prime}\right|^{2}} \frac{\partial^{2} v}{\partial s^{2}}+H \frac{\partial v}{\partial \varepsilon}\right](h \cdot \nu)
\end{aligned}
$$

or rearranging the terms

$$
\begin{aligned}
G_{N}^{\prime}(z)= & -k^{2}(h \cdot \nu) v+H(h \cdot \nu) \frac{\partial v}{\partial \varepsilon}+\frac{(h \cdot \tau)}{\left|z^{\prime}\right|} \frac{\partial^{2} v}{\partial s \partial \varepsilon} \\
& -\frac{1}{\left|z^{\prime}\right|^{2}}\left[h^{\prime} \cdot \nu-(h \cdot \nu) \frac{z^{\prime} \cdot z^{\prime \prime}}{\left|z^{\prime}\right|^{2}}+(h \cdot \tau) H\left|z^{\prime}\right|\right] \frac{\partial v}{\partial s}-\frac{h \cdot \nu}{\left|z^{\prime}\right|^{2}} \frac{\partial^{2} v}{\partial s^{2}}
\end{aligned}
$$

where for simplicity $v$ holds for $v(s, 0)$.

Considering (3.30) one gets

$$
\begin{aligned}
\frac{\partial}{\partial \tau}\left(h \cdot \nu\left(\frac{\partial u}{\partial \tau} \circ z\right)\right)= & \frac{1}{\left|z^{\prime}\right|^{2}}\left[h^{\prime} \cdot \nu+h \cdot \nu^{\prime}-(h \cdot \nu) \frac{z^{\prime} \cdot z^{\prime \prime}}{\left|z^{\prime}\right|^{2}}\right] \frac{\partial v}{\partial s} \\
& +\frac{(h \cdot \nu)}{\left|z^{\prime}\right|^{2}} \frac{\partial^{2} v}{\partial s^{2}}
\end{aligned}
$$

and if one has the identity

$$
h \cdot \nu^{\prime}=-\frac{(h \cdot \tau)}{\left|z^{\prime}\right|}\left(\nu \cdot z^{\prime \prime}\right)=(h \cdot \tau) H\left|z^{\prime}\right|
$$

one can substitute (3.35) in (3.34) and the latter in (3.33), obtaining the result by (3.29) and (3.30).

To prove $(3.35$ ) one starts by noting that

$$
h \cdot \nu^{\prime}=h \cdot\left(\frac{z^{\prime \prime \perp}}{\left|z^{\prime}\right|}-\frac{z^{\prime \prime} \cdot z^{\prime}}{\left|z^{\prime}\right|^{2}} \nu\right)=-\frac{h^{\perp} \cdot z^{\prime \prime}}{\left|z^{\prime}\right|}-(h \cdot \nu) \frac{z^{\prime \prime} \cdot \tau}{\left|z^{\prime}\right|} .
$$

Now one only needs to decompose $h$ in its tangential and normal component in order to get

$$
h^{\perp}=(h \cdot \tau) \nu-(h \cdot \nu) \tau
$$

and apply it in (3.36). 
With this characterization, the linearized equation (3.20) can be rewritten in the following way

$$
\left((h \cdot \nu)\left(H \frac{\partial u_{n}}{\partial \nu}-k^{2} u_{n}\right)-\frac{\partial}{\partial \tau}\left(h \cdot \nu\left(\frac{\partial u_{n}}{\partial \tau}\right)\right)+(h \cdot \tau) \frac{\partial^{2} u_{n}}{\partial \tau \partial \nu}\right) \circ z_{n}=-\frac{\partial u_{n}}{\partial \nu} \circ z_{n}
$$

in $[0,2 \pi]$. In this way, at each iteration $n$ we solve the previous equation with respect to $h$ in a least squares sense, using the approximated scattered field $u_{n}^{s}$ as obtained in the first step of the method to approximate the total field in the previous equation by $u_{n}=u^{i}+u_{n}^{s}$. We then update the approximation for the domain, getting $\gamma_{n+1}$ parameterized by $z_{n+1}=z_{n}+h$. Note that again we use the jump relations (3.16) and (3.17) and the relation (3.30) to compute the terms involved. The details on the numerical implementation are presented in section 5.1.3.

\section{Three-dimensional Case}

Though we did not implement the hybrid method for the Neumann boundary condition in $\mathbb{R}^{3}$, we will now study the characterization of $G_{N}^{\prime}$ for the three-dimensional case. The procedure is similar to the two-dimensional case, with the difference that now the tangential space has dimension two.

We recall that we consider a parameterization $z:[0, \pi] \times[0,2 \pi] \rightarrow \mathbb{R}^{3}$. Therefore the corresponding surface $\gamma$ is given by

$$
\gamma_{z}=\{z(\theta, \phi):(\theta, \phi) \in[0, \pi] \times[0,2 \pi]\} .
$$

On $\gamma$ we define two orthogonal unit tangential vector fields $\tau_{1}$ and $\tau_{2}$. As a parameterized characterization of $G_{N}^{\prime}$ is of use for the numerical implementation later on, we will consider

$$
\tau_{1}=\frac{z_{\theta}}{\left|z_{\theta}\right|}, \quad \tau_{2}=\frac{z_{\phi}-\left(z_{\phi} \cdot z_{\theta}\right) z_{\theta}}{\left|z_{\phi}-\left(z_{\phi} \cdot z_{\theta}\right) z_{\theta}\right|}
$$

where $z_{\theta}=\partial z / \partial \theta$ and $z_{\phi}=\partial z / \partial \phi$, but the following analysis holds for any other two orthonormal tangential vector fields $\tau_{1}$ and $\tau_{2}$. We will also introduce as notation for the non-normalized tangential vectors

$$
\tilde{\tau}_{1}=z_{\theta}, \quad \tilde{\tau}_{2}=z_{\phi}-\left(z_{\phi} \cdot z_{\theta}\right) z_{\theta} .
$$

We can now write the operator $G_{N}$ as

$$
G_{N}: z \mapsto\left(\frac{\tau_{1} \times \tau_{2}}{\left|\tau_{1} \times \tau_{2}\right|}\right) \cdot \operatorname{grad} u \circ z .
$$

where $\times$ is the usual cross product. We will assume without loss of generality that

$$
\nu=\frac{\tau_{1} \times \tau_{2}}{\left|\tau_{1} \times \tau_{2}\right|}
$$


is the exterior normal to $\gamma_{z}$ (otherwise we would just need to change the rolls of $\tau_{1}$ and $\tau_{2}$ ), where for simplicity we denote $\nu=\nu \circ z$ and $\tau_{i}=\tau_{i} \circ z$ for $i=1,2$.

The analog of corollary 3.12 in three dimensions is the following theorem.

Theorem 3.13. The operator $G_{N}: C^{2}([0, \pi] \times[0,2 \pi]) \rightarrow C([0, \pi] \times[0,2 \pi])$ is Fréchet differentiable and provided that u satisfies the Helmholtz equation its Fréchet derivative is given by

$$
\begin{aligned}
G_{N}^{\prime}(z) h= & {\left[-k^{2}(h \cdot \nu) u-\operatorname{Div}\left((h \cdot \nu) \nabla_{\tau} u\right)+h \cdot\left(\nabla_{\tau} \frac{\partial u}{\partial \nu}\right)\right.} \\
& \left.+2 H(h \cdot \nu) \frac{\partial u}{\partial \nu}\right] \circ z
\end{aligned}
$$

in $[0, \pi] \times[0,2 \pi]$, where $H$ stands for the mean curvature and Div is the tangential divergence.

Proof. The Fréchet differentiability of $G_{N}$ is again a direct consequence of the analyticity of $u$ and the $C^{2}$-smoothness of $z$. Let $h$ be sufficiently small to ensure that

$$
\gamma_{z+h}=\{z(\theta, \phi)+h(\theta, \phi):(\theta, \phi) \in[0, \pi] \times[0,2 \pi]\}
$$

describes a closed surface. Again we will do a pointwise analysis to characterize the Fréchet derivative $G_{N}^{\prime}$. In this way, we use the characterization for the exterior normal given by

$$
\nu=\frac{z_{\theta} \times z_{\phi}}{\left|z_{\theta} \times z_{\phi}\right|}
$$

As in the two-dimensional case, one uses the decomposition

$$
\begin{aligned}
G_{N}(z+h)-G_{N}(z)= \\
=\left(\frac{(z+h)_{\theta} \times(z+h)_{\phi}}{\left|(z+h)_{\theta} \times(z+h)_{\phi}\right|}-\frac{z_{\theta} \times z_{\phi}}{\left|z_{\theta} \times z_{\phi}\right|}\right) \cdot(\operatorname{grad} u \circ(z+h)) \\
\quad+\left(\frac{z_{\theta} \times z_{\phi}}{\left|z_{\theta} \times z_{\phi}\right|}\right) \cdot(\operatorname{grad} u \circ(z+h)-\operatorname{grad} u \circ z)
\end{aligned}
$$

and treat both terms on the right hand side separately. Using Taylor's formula, we begin by noting that

$$
\begin{aligned}
& \left(\frac{(z+h)_{\theta} \times(z+h)_{\phi}}{\left|(z+h)_{\theta} \times(z+h)_{\phi}\right|}-\frac{z_{\theta} \times z_{\phi}}{\left|z_{\theta} \times z_{\phi}\right|}\right)= \\
& =\frac{1}{\left|z_{\theta} \times z_{\phi}\right|}\left[h_{\theta} \times z_{\phi}+z_{\theta} \times h_{\phi}-\nu\left(\left(z_{\phi} \times \nu\right) \cdot h_{\theta}+\left(\nu \times z_{\theta}\right) \cdot h_{\phi}\right)\right] \\
& \quad+O\left(\|\left. h\right|_{C^{2}} ^{2}\right)
\end{aligned}
$$


Using

$$
\operatorname{grad} u \circ(z+h)-\operatorname{grad} u \circ z=O(|h|)
$$

we consequently have

$$
\begin{aligned}
& \left(\frac{(z+h)_{\theta} \times(z+h)_{\phi}}{\left|(z+h)_{\theta} \times(z+h)_{\phi}\right|}-\frac{z_{\theta} \times z_{\phi}}{\left|z_{\theta} \times z_{\phi}\right|}\right) \cdot(\operatorname{grad} u \circ(z+h))= \\
& =\frac{1}{\left|z_{\theta} \times z_{\phi}\right|}\left[h_{\theta} \times z_{\phi}+z_{\theta} \times h_{\phi}-\nu\left(\left(z_{\phi} \times \nu\right) \cdot h_{\theta}+\left(\nu \times z_{\theta}\right) \cdot h_{\phi}\right)\right] \cdot \operatorname{grad} u \circ z \\
& \quad+O\left(\|\left. h\right|_{C^{2}} ^{2}\right) .
\end{aligned}
$$

In order to perform a change of variables we need an orthogonal coordinate system at each point of the surface $\gamma$. Since the analysis is done pointwise, we consider a fixed but arbitrary point $x_{0}=z\left(\theta_{0}, \phi_{0}\right) \in \gamma$. Without loss of generality, we will assume that $z_{\theta} \perp z_{\phi}$ at $\left(\theta_{0}, \phi_{0}\right)$, that is, that $z_{\theta} \cdot z_{\phi}=0$. Note that if this is not the case one can locally choose a parameterization $\tilde{z}(\theta, \phi)$ of $\gamma$ in a neighbourhood of $x_{0}$ such that on has $x_{0}=\tilde{z}\left(\theta_{0}, \phi_{0}\right)$ and the variable $\theta$ corresponds to the direction $\tilde{\tau}_{1}$ and $\phi$ to the direction $\tilde{\tau}_{2}$, for $\tilde{\tau}_{1}$ and $\tilde{\tau}_{2}$ given by (3.39). In other words, we would pick a parameterization $\tilde{z}$ such that $\tilde{z}_{\theta}=\tilde{\tau}_{1}$ and $\tilde{z}_{\phi}=\tilde{\tau}_{2}$. Note that as the analysis is pointwise this would not influence the final result since $\tilde{z}\left(\theta_{0}, \phi_{0}\right)=x_{0}=z\left(\theta_{0}, \phi_{0}\right)$ and the point $x_{0}$ was fixed but arbitrary.

We are now in a position to perform a change of variables in a neighbourhood of $x_{0} \in \gamma$ by

$$
x(\theta, \phi, \varepsilon)=z(\theta, \phi)+\varepsilon \nu(\theta, \phi),
$$

and set

$$
v(\theta, \phi, \varepsilon)=u(z(\theta, \phi)+\varepsilon \nu(\theta, \phi))
$$

in order to get in the new coordinate system (e.g [62])

$$
\begin{aligned}
\operatorname{grad} v(\theta, \phi, \varepsilon)= & \frac{1}{\left|z_{\theta}(\theta, \phi)+\varepsilon \nu_{\theta}(\theta, \phi)\right|^{2}} \frac{\partial v}{\partial \theta}(\theta, \phi, \varepsilon)\left[z_{\theta}(\theta, \phi)+\varepsilon \nu_{\theta}(\theta, \phi)\right] \\
& +\frac{1}{\left|z_{\phi}(\theta, \phi)+\varepsilon \nu_{\phi}(\theta, \phi)\right|^{2}} \frac{\partial v}{\partial \phi}(\theta, \phi, \varepsilon)\left[z_{\phi}(\theta, \phi)+\varepsilon \nu_{\phi}(\theta, \phi)\right] \\
& +\frac{\partial v}{\partial \varepsilon}(\theta, \phi, \varepsilon) \nu(s) .
\end{aligned}
$$

Therefore, using the relations $\nu \cdot z_{\theta}=\nu \cdot z_{\phi}=0$ and some properties of the cross product such as $(a \times b) \cdot a=0$ for all $a, b \in \mathbb{R}^{3}$ and $a \cdot(b \times c)=(a \times b) \cdot c$ for all $a, b, c \in \mathbb{R}^{3}$ to conclude that

$$
\left(h_{\theta} \times z_{\phi}\right) \cdot \nu=h_{\theta} \cdot\left(z_{\phi} \times \nu\right), \quad\left(h_{\phi} \times z_{\theta}\right) \cdot \nu=h_{\phi} \cdot\left(z_{\theta} \times \nu\right),
$$


we can rewrite (3.42) as

$$
\begin{aligned}
& \left(\frac{(z+h)_{\theta} \times(z+h)_{\phi}}{\left|(z+h)_{\theta} \times(z+h)_{\phi}\right|}-\frac{z_{\theta} \times z_{\phi}}{\left|z_{\theta} \times z_{\phi}\right|}\right) \cdot(\operatorname{grad} u \circ(z+h))= \\
& \quad=-\frac{\left(h_{\theta} \cdot \nu\right)}{\left|z_{\theta}\right|^{2}} \frac{\partial v}{\partial \theta}-\frac{\left(h_{\phi} \cdot \nu\right)}{\left|z_{\phi}\right|^{2}} \frac{\partial v}{\partial \phi}+O\left(\|h\|_{C^{2}}^{2}\right)
\end{aligned}
$$

where the evaluation point $x_{0}=z\left(\theta_{0}, \phi_{0}\right)$ is omitted in the previous and following equations to ease the notation.

We now consider the second term on the right hand side of (3.41). Taylor's formula and the relations $\nu \cdot z_{\theta}=\nu \cdot z_{\phi}=0$ and $\nu \cdot \nu_{\theta}=\nu \cdot \nu_{\phi}=0$ imply that

$$
\begin{aligned}
\nu(\theta, \phi) \cdot[\operatorname{grad} v(\theta+\sigma, \phi+\xi, \epsilon)-\operatorname{grad} v(\theta, \phi, 0)] \\
=\left[\frac{z_{\theta \theta} \cdot \nu}{\left|z_{\theta}\right|^{2}} \frac{\partial v}{\partial \theta}(\theta, \phi, 0)+\frac{z_{\phi \theta} \cdot \nu}{\left|z_{\phi}\right|^{2}} \frac{\partial v}{\partial \phi}(\theta, \phi, 0)+\frac{\partial^{2} v}{\partial \theta \partial \epsilon}(\theta, \phi, 0)\right] \sigma \\
+\left[\frac{z_{\phi \phi} \cdot \nu}{\left|z_{\phi}\right|^{2}} \frac{\partial v}{\partial \phi}(\theta, \phi, 0)+\frac{z_{\theta \phi} \cdot \nu}{\left|z_{\theta}\right|^{2}} \frac{\partial v}{\partial \theta}(\theta, \phi, 0)+\frac{\partial^{2} v}{\partial \phi \partial \epsilon}(\theta, \phi, 0)\right] \xi \\
+\frac{\partial^{2} v}{\partial \varepsilon^{2}}(\theta, \phi, 0) \epsilon .
\end{aligned}
$$

In view of the second term on the right hand side of (3.41) we again want to choose the triplet $(\sigma, x i, \epsilon)$ such that

$$
z\left(\theta_{0}, \phi_{0}\right)+h\left(\theta_{0}, \phi_{0}\right)=z\left(\theta_{0}+\sigma, \phi_{0}+\xi\right)+\epsilon \nu\left(\theta_{0}+\sigma, \phi_{0}+\xi\right)
$$

and (similarly to the two-dimensional case) by Taylor's formula we get

$$
\sigma=\frac{h \cdot z_{\theta}}{\left|z_{\theta}\right|^{2}}, \quad \xi=\frac{h \cdot z_{\phi}}{\left|z_{\phi}\right|^{2}}, \quad \text { and } \quad \epsilon=h \cdot \nu
$$

In this way, we get

$$
\begin{aligned}
G_{N}(z+h)-G_{N}(z)= & -\frac{\left(h_{\theta} \cdot \nu\right)}{\left|z_{\theta}\right|^{2}} \frac{\partial v}{\partial \theta}-\frac{\left(h_{\phi} \cdot \nu\right)}{\left|z_{\phi}\right|^{2}} \frac{\partial v}{\partial \phi} \\
& +\left[\frac{z_{\theta \theta} \cdot \nu}{\left|z_{\theta}\right|^{2}} \frac{\partial v}{\partial \theta}+\frac{z_{\phi \theta} \cdot \nu}{\left|z_{\phi}\right|^{2}} \frac{\partial v}{\partial \phi}+\frac{\partial^{2} v}{\partial \theta \partial \epsilon}\right] \frac{h \cdot z_{\theta}}{\left|z_{\theta}\right|^{2}} \\
& +\left[\frac{z_{\phi \phi} \cdot \nu}{\left|z_{\phi}\right|^{2}} \frac{\partial v}{\partial \phi}+\frac{z_{\theta \phi} \cdot \nu}{\left|z_{\theta}\right|^{2}} \frac{\partial v}{\partial \theta}+\frac{\partial^{2} v}{\partial \phi \partial \epsilon}\right] \frac{h \cdot z_{\phi}}{\left|z_{\phi}\right|^{2}} \\
& +\frac{\partial^{2} v}{\partial \varepsilon^{2}} h \cdot \nu+O\left(\|h\|_{C^{2}}^{2}\right) .
\end{aligned}
$$


For numerical purposes we want to get rid of the term $\partial^{2} v / \partial \varepsilon^{2}$. Therefore one uses the fact that the Laplacian is given in the new coordinate system (e.g [62]) by

$$
\begin{aligned}
\Delta v(., \varepsilon)= & \frac{1}{\left|z_{\theta}(.)+\varepsilon \nu_{\theta}(.)\right|\left|z_{\theta}(.)+\varepsilon \nu_{\phi}(.)\right|}\left[\frac{\partial}{\partial \theta}\left(\frac{\left|z_{\theta}(.)+\varepsilon \nu_{\phi}(.)\right|}{\left|z_{\theta}(.)+\varepsilon \nu_{\theta}(.)\right|} \frac{\partial v}{\partial \theta}(., \varepsilon)\right)\right. \\
& +\frac{\partial}{\partial \phi}\left(\frac{\left|z_{\theta}(.)+\varepsilon \nu_{\theta}(.)\right|}{\left|z_{\theta}(.)+\varepsilon \nu_{\phi}(.)\right|} \frac{\partial v}{\partial \phi}(., \varepsilon)\right) \\
& \left.+\frac{\partial}{\partial \varepsilon}\left(\left|z_{\theta}(.)+\varepsilon \nu_{\phi}(.)\right|\left|z_{\theta}(.)+\varepsilon \nu_{\theta}(.)\right| \frac{\partial v}{\partial \varepsilon}(., \varepsilon)\right)\right]
\end{aligned}
$$

and therefore taking the limit as $\varepsilon \rightarrow 0$ we get

$$
\begin{aligned}
\Delta v \rightarrow & \left(\frac{z_{\phi \theta} \cdot z_{\phi}}{\left|z_{\theta}\right|^{2}\left|z_{\phi}\right|^{2}}-\frac{z_{\theta \theta} \cdot z_{\theta}}{\left|z_{\theta}\right|^{4}}\right) \frac{\partial v}{\partial \theta}+\left(\frac{z_{\theta \phi} \cdot z_{\theta}}{\left|z_{\theta}\right|^{2}\left|z_{\phi}\right|^{2}}-\frac{z_{\phi \phi} \cdot z_{\phi}}{\left|z_{\phi}\right|^{4}}\right) \frac{\partial v}{\partial \phi} \\
& +\frac{1}{\left|z_{\theta}\right|^{2}} \frac{\partial^{2} v}{\partial \theta^{2}}+\frac{1}{\left|z_{\phi}\right|^{2}} \frac{\partial^{2} v}{\partial \phi^{2}}+\left(\frac{z_{\theta} \cdot \nu_{\theta}}{\left|z_{\theta}\right|^{2}}+\frac{z_{\phi} \cdot \nu_{\phi}}{\left|z_{\phi}\right|^{2}}\right) \frac{\partial v}{\partial \varepsilon}+\frac{\partial^{2} v}{\partial \varepsilon^{2}} .
\end{aligned}
$$

As $u$ satisfies the Helmholtz equation one gets

$$
\begin{aligned}
G_{N}(z+h)-G_{N}(z)= & -\frac{\left(h_{\theta} \cdot \nu\right)}{\left|z_{\theta}\right|^{2}} \frac{\partial v}{\partial \theta}-\frac{\left(h_{\phi} \cdot \nu\right)}{\left|z_{\phi}\right|^{2}} \frac{\partial v}{\partial \phi} \\
+ & {\left[\frac{z_{\theta \theta} \cdot \nu}{\left|z_{\theta}\right|^{2}} \frac{\partial v}{\partial \theta}+\frac{z_{\phi \theta} \cdot \nu}{\left|z_{\phi}\right|^{2}} \frac{\partial v}{\partial \phi}+\frac{\partial^{2} v}{\partial \theta \partial \epsilon}\right] \frac{h \cdot z_{\theta}}{\left|z_{\theta}\right|^{2}} } \\
+ & {\left[\frac{z_{\phi \phi} \cdot \nu}{\left|z_{\phi}\right|^{2}} \frac{\partial v}{\partial \phi}+\frac{z_{\theta \phi} \cdot \nu}{\left|z_{\theta}\right|^{2}} \frac{\partial v}{\partial \theta}+\frac{\partial^{2} v}{\partial \phi \partial \epsilon}\right] \frac{h \cdot z_{\phi}}{\left|z_{\phi}\right|^{2}} } \\
- & {\left[k^{2} v+\left(\frac{z_{\phi \theta} \cdot z_{\phi}}{\left|z_{\theta}\right|^{2}\left|z_{\phi}\right|^{2}}-\frac{z_{\theta \theta} \cdot z_{\theta}}{\left|z_{\theta}\right|^{4}}\right) \frac{\partial v}{\partial \theta}\right.} \\
& +\left(\frac{z_{\theta \phi} \cdot z_{\theta}}{\left|z_{\theta}\right|^{2}\left|z_{\phi}\right|^{2}}-\frac{z_{\phi \phi} \cdot z_{\phi}}{\left|z_{\phi}\right|^{4}}\right) \frac{\partial v}{\partial \phi}+\frac{1}{\left|z_{\theta}\right|^{2}} \frac{\partial^{2} v}{\partial \theta^{2}}+\frac{1}{\left|z_{\phi}\right|^{2}} \frac{\partial^{2} v}{\partial \phi^{2}} \\
& \left.+\left(\frac{z_{\theta} \cdot \nu_{\theta}}{\left|z_{\theta}\right|^{2}}+\frac{z_{\phi} \cdot \nu_{\phi}}{\left|z_{\phi}\right|^{2}}\right) \frac{\partial v}{\partial \varepsilon}\right] h \cdot \nu+O\left(\|h\|_{C^{2}}^{2}\right) . \quad(3.45)
\end{aligned}
$$

By straight forward computations, using the fact that

$$
\nu_{\theta}=\frac{1}{\left|z_{\theta} \times z_{\phi}\right|}\left(\left(z_{\theta \theta} \times z_{\phi}\right)+\left(z_{\theta} \times z_{\phi \theta}\right)+\nu\left(\left(\nu \times z_{\phi}\right) \cdot z_{\theta \theta}+\left(z_{\theta} \times \nu\right) \cdot z_{\phi \theta}\right)\right)
$$


one concludes that

$$
h \cdot \nu_{\theta}=-\left(\frac{\left(h \cdot z_{\theta}\right)}{\left|z_{\theta}\right|^{2}}\left(\nu \cdot z_{\theta \theta}\right)+\frac{\left(h \cdot z_{\phi}\right)}{\left|z_{\phi}\right|^{2}}\left(\nu \cdot z_{\phi \theta}\right)\right) .
$$

In this way, collecting all the terms in $\partial v / \partial \theta$ from (3.45) we get that

$$
\begin{gathered}
\left(-\frac{\left(h_{\theta} \cdot \nu\right)}{\left|z_{\theta}\right|^{2}}+\frac{\left(z_{\theta \theta} \cdot \nu\right)\left(h \cdot z_{\theta}\right)}{\left|z_{\theta}\right|^{4}}+\frac{\left(z_{\theta \phi} \cdot \nu\right)\left(h \cdot z_{\phi}\right)}{\left|z_{\theta}\right|^{2}\left|z_{\phi}\right|^{2}}-\frac{z_{\phi \theta} \cdot z_{\phi}}{\left|z_{\theta}\right|^{2}\left|z_{\phi}\right|^{2}}+\frac{z_{\theta \theta} \cdot z_{\theta}}{\left|z_{\theta}\right|^{4}}\right) \frac{\partial v}{\partial \theta}= \\
=\frac{1}{\left|z_{\theta}\right|\left|z_{\phi}\right|}\left(-\frac{\left|z_{\phi}\right|}{\left|z_{\theta}\right|}\left(\left(h_{\theta} \cdot \nu\right)+\left(h \cdot \nu_{\theta}\right)\right)-\frac{z_{\phi \theta} \cdot z_{\phi}}{\left|z_{\phi}\right|\left|z_{\theta}\right|}+\frac{\left(z_{\theta \theta} \cdot z_{\theta}\right)\left|z_{\phi}\right|}{\left|z_{\theta}\right|^{3}}\right) \frac{\partial v}{\partial \theta} \\
=-\frac{1}{\left|z_{\theta}\right|\left|z_{\phi}\right|} \frac{\partial}{\partial \theta}\left((h \cdot \nu) \frac{\left|z_{\phi}\right|}{\left|z_{\theta}\right|}\right) \frac{\partial v}{\partial \theta} .
\end{gathered}
$$

Now summing the term in $\partial^{2} v / \partial \theta^{2}$ from (3.45) we get

$$
-\frac{1}{\left|z_{\theta}\right|\left|z_{\phi}\right|} \frac{\partial}{\partial \theta}\left((h \cdot \nu) \frac{\left|z_{\phi}\right|}{\left|z_{\theta}\right|}\right) \frac{\partial v}{\partial \theta}-\frac{h \cdot \nu}{\left|z_{\theta}\right|^{2}} \frac{\partial^{2} v}{\partial \theta^{2}}=-\frac{1}{\left|z_{\theta}\right|\left|z_{\phi}\right|} \frac{\partial}{\partial \theta}\left((h \cdot \nu) \frac{\left|z_{\phi}\right|}{\left|z_{\theta}\right|} \frac{\partial v}{\partial \theta}\right) .
$$

Interchanging the roles of $\theta$ and $\phi$ we can write all the terms in the first or second order derivative of $v$ in $\theta$ and $\phi$ from (3.45) in the simplified form

$$
-\frac{1}{\left|z_{\theta}\right|\left|z_{\phi}\right|}\left[\frac{\partial}{\partial \theta}\left((h \cdot \nu) \frac{\left|z_{\phi}\right|}{\left|z_{\theta}\right|} \frac{\partial v}{\partial \theta}\right)+\frac{\partial}{\partial \phi}\left((h \cdot \nu) \frac{\left|z_{\theta}\right|}{\left|z_{\phi}\right|} \frac{\partial v}{\partial \phi}\right)\right]=-\operatorname{Div}\left((h \cdot \nu) \nabla_{\tau} u\right)
$$

where $\nabla_{\tau}$ denotes the tangential gradient of $u$. The previous equality comes from the fact that in the new coordinate system the tangential divergence over the surface $\gamma$ of a tangential field $A$ is given by (e.g. [62])

$$
\operatorname{Div} A=\frac{1}{\left|z_{\theta}\right|\left|z_{\phi}\right|} \quad\left[\frac{\partial}{\partial \theta}\left(\frac{\left|z_{\phi}\right|}{\left|z_{\theta}\right|}\left(A \cdot z_{\theta}\right)\right)+\frac{\partial}{\partial \phi}\left(\frac{\left|z_{\theta}\right|}{\left|z_{\phi}\right|}\left(A \cdot z_{\phi}\right)\right)\right]
$$

and from the equalities

$$
\frac{\partial v}{\partial \varepsilon}(\theta, \phi, 0)=\frac{\partial u}{\partial \nu}(z(\theta, \phi))
$$

and

$$
\begin{aligned}
& \frac{1}{\left|z_{\theta}(\theta, \phi)\right|} \frac{\partial v}{\partial \theta}(\theta, \phi, 0)=\frac{\partial u}{\partial \tau_{1}}(z(\theta, \phi)), \\
& \frac{1}{\left|z_{\phi}(\theta, \phi)\right|} \frac{\partial v}{\partial \phi}(\theta, \phi, 0)=\frac{\partial u}{\partial \tau_{2}}(z(\theta, \phi)) .
\end{aligned}
$$


Finally, applying (3.46), (3.47) and (3.48) to the remaining terms of (3.45) one gets the result from the definition of Fréchet derivative and from the definition of mean curvature $H$ of a surface in $\mathbb{R}^{3}$ as

$$
H=-\frac{\tilde{\nu} \cdot\left(\left(\tilde{\nu}_{\theta} \times z_{\phi}\right)+\left(z_{\theta} \times \tilde{\nu}_{\phi}\right)\right)}{2|\tilde{\nu}|^{3}}=-\frac{1}{2}\left(\tau_{1} \cdot \frac{\partial \nu}{\partial \tau_{1}}+\tau_{2} \cdot \frac{\partial \nu}{\partial \tau_{2}}\right)
$$

with $\tilde{\nu}=z_{\theta} \times z_{\phi}$.

Again we would use the characterization (3.40) for $G_{N}^{\prime}$ in (3.20) and solve this equation (using $u_{n}$ as approximation to the total field) with respect to $h$ in a least squares sense, in order to obtain the new approximation $\gamma_{n+1}$ parameterized by $z_{n+1}=z_{n}+h$. To compute the terms involved, we use the jump relations (3.16) and (3.17), along with the relations

$$
\frac{\partial u}{\partial \tau_{1}} \circ z=\frac{1}{\left|z_{\theta}\right|} \frac{\partial v}{\partial \theta}
$$

and

$$
\frac{\partial u}{\partial \tau_{2}} \circ z=\frac{1}{z_{\phi} \cdot \tau_{2}}\left(\frac{\partial v}{\partial \phi}-\frac{z_{\phi} \cdot \tau_{1}}{\left|z_{\theta}\right|} \frac{\partial v}{\partial \theta}\right)=\frac{1}{\left|z_{\phi}\right|^{2}-\left(z_{\phi} \cdot z_{\theta}\right)^{2}}\left(\frac{\partial v}{\partial \phi}-\frac{\left(z_{\phi} \cdot z_{\theta}\right)}{\left|z_{\theta}\right|^{2}} \frac{\partial v}{\partial \theta}\right)
$$

in order to compute the tangential gradient given by

$$
\nabla_{\tau} u=\frac{\partial u}{\partial \tau_{1}} \tau_{1}+\frac{\partial u}{\partial \tau_{2}} \tau_{2}
$$

\subsection{The Hybrid Method for the Robin Case}

We will now combine both previous approaches for the Dirichlet and Neumann cases in order to solve the inverse problem 2.15 with Robin boundary condition. The solution $u^{s}$ to the direct problem must satisfy

$$
\begin{array}{r}
\Delta u^{s}+k^{2} u^{s}=0, \quad x \in \mathbb{R}^{m} \backslash \bar{D} \\
\frac{\partial u}{\partial \nu}+i \lambda u=0, \quad x \in \Gamma, \\
\lim _{r \rightarrow \infty} r^{\frac{m-1}{2}}\left(\frac{\partial u^{s}}{\partial r}-i k u^{s}\right)=0,
\end{array}
$$

where again the total field $u$ is the sum of the given incident field $u^{i}$ and the scattered field $u^{s}$. Now the goal is not only to recover the domain $D$ but also the unknown impedance $\lambda$, from the knowledge of the far-field pattern $u_{\infty}$ for one incident plane 
wave $u^{i}(x)=e^{i k x \cdot d},|d|=1$. In this way, at each iteration we consider to have both an approximation $\gamma_{n}$ (parameterized by $z_{n}$ ) to the correct boundary $\Gamma$, as well as an approximation $\zeta_{n}$ to the correct impedance $\lambda$.

In the first step of the iteration, that is, to reconstruct the total field $u$, the procedure will be exactly the same as for the Neumann case. Having in mind assumption 3.2, in order to get a meaningful representation of the scattered field $u^{s}$ as a layer potential over $\gamma_{n}$, we need to assume that the solution $u^{s}$ to the direct problem of scattering by $D$ with impedance $\lambda$ can be analytically extended until $\gamma_{n}$. Analogously to remark 3.3, for the Robin case one has to require that both the boundary $\Gamma$ and the impedance $\lambda$ are analytic in order to guarantee that the analytic continuation principle 3.2 holds.

Therefore we represent the scattered field $u^{s}$ as a combined single-and double-layer potential over $\gamma_{n}$ (see [8, thm.3.38]), that is,

$$
u^{s}(x)=\int_{\gamma_{n}}\left(\frac{\partial \Phi(x, y)}{\partial \nu(y)}-i \eta \Phi(x, y)\right) \varphi(y) d s(y), \quad x \in \mathbb{R}^{m} \backslash \gamma_{n}
$$

for some coupling parameter $\eta>0$ and with density $\varphi \in C^{1, \alpha}\left(\gamma_{n}\right)$. All the remarks made on this representation in the previous section concerning the Neumann problem remain true. For instance we have that the exterior trace of $u^{s}$ over $\gamma$ is given by (3.16) and the normal trace of $u^{s}$ is given by (3.17). In this way the impedance trace of $u^{s}$ over $\gamma$ with impedance $\zeta$ is given by

$$
\frac{\partial u^{s}}{\partial \nu}+i \zeta u^{s}=B_{\gamma, \zeta} \varphi \quad \text { on } \gamma
$$

where

$$
B_{\gamma, \zeta}=i(\eta+\zeta) \frac{I}{2}+T_{\gamma}+i\left(\zeta K_{\gamma}-\eta K_{\gamma}^{*}\right)+\zeta \eta S_{\gamma}
$$

is the exterior impedance trace operator $B_{\gamma, \zeta}: C^{1, \alpha}(\gamma) \rightarrow C(\gamma)$ of the combined singleand double-layer potential.

Again, by the asymptotics of the potentials, the equation

$$
F_{\gamma, \infty} \varphi=u_{\infty} \quad \text { on } \Omega_{m}
$$

must be satisfied with $F_{\gamma, \infty}:=\left(K_{\gamma, \infty}-i \eta S_{\gamma, \infty}\right)$, where the far-field single-and doublelayer operators $S_{\gamma, \infty}, K_{\gamma, \infty}: C^{1, \alpha}\left(\gamma_{n}\right) \rightarrow C\left(\gamma_{n}\right)$ are defined in (2.22) and (2.23). As the operator $F_{\gamma, \infty}$ is compact, we use Tikhonov regularization to get the regularized equation

$$
\left(\alpha_{n} I+F_{\gamma, \infty}^{*} F_{\gamma, \infty}\right) \varphi^{(n)}=F_{\gamma, \infty}^{*} u_{\infty}
$$

and solve it with respect to $\varphi^{(n)}$ for some regularization parameter $\alpha_{n}>0$ decreasing with the iteration $n$. In this way we construct an approximation to the scattered field given by

$$
u_{n}^{s}(x)=\int_{\gamma_{n}}\left(\frac{\partial \Phi(x, y)}{\partial \nu(y)}-i \eta \Phi(x, y)\right) \varphi^{(n)}(y) d s(y), \quad x \in \mathbb{R}^{m} \backslash \gamma_{n}
$$


and by the jump relations we have approximations to the exterior trace and normal trace of $u$ given by

$$
\begin{array}{r}
u_{n}^{s}=\frac{\varphi^{(n)}}{2}+\left(K_{\gamma_{n}}-i \eta S_{\gamma_{n}}\right) \varphi^{(n)} \quad \text { on } \gamma_{n}, \\
\frac{\partial u_{n}^{s}}{\partial \nu}=i \eta \frac{\varphi^{(n)}}{2}+\left(T_{\gamma_{n}}-i \eta K_{\gamma_{n}}^{*}\right) \varphi^{(n)} \quad \text { on } \gamma_{n} .
\end{array}
$$

As for the second step, we start by defining the operator $G_{R}$ that maps the pair $(z, \zeta)$ where $z$ is the parameterization of the contour $\gamma$ to the exterior trace of the Robin boundary condition with impedance $\zeta$ of some fixed field $u$ over $\gamma$, that is,

$$
G_{R}:(z, \zeta) \mapsto(\nu \cdot \operatorname{grad} u+i \zeta u) \circ z .
$$

Remark 3.14. Note that the function $\zeta$ is only defined in the boundary $\gamma$, from its definition as an impedance function. However, $\zeta$ must be defined in all $\mathbb{R}^{m}$ so that the previous operator is defined for all parameterizations $z$. Since we are just interested in a local characterization around $z_{n}$, we will need to extend $\zeta_{n}$ in a proper way around $\gamma_{n}$. We will address this extension later at the beginning of section 5.1.4 and for the time being we will just assume that this can be done.

One also has that $G_{R}$ can be given in terms of $G_{D}$ and $G_{N}$ by

$$
G_{R}(z, \zeta)=G_{N}(z)+i \zeta G_{D}(z)
$$

If the fixed field $u$ would be the exact total field, having in view the boundary condition, we would like to look for a solution to

$$
G_{R}(z, \zeta)=0
$$

and again based on the Fréchet differentiability of $G_{R}$, we linearize the previous equation around $z_{n}$ and $\zeta_{n}$ and solve the linearized equation

$$
G_{R}\left(z_{n}, \zeta_{n}\right)+\frac{\partial}{\partial z} G_{R}\left(z_{n}, \zeta_{n}\right) h+\frac{\partial}{\partial \zeta} G_{R}\left(z_{n}, \zeta_{n}\right) \mu=0 \quad \text { in } X
$$

in terms of the shifts $h$ and $\mu$. A characterization for the Fréchet derivative of $G_{N}$ is now needed and can be easily obtained using the characterizations of $G_{D}^{\prime}$ and $G_{N}^{\prime}$ given in the two previous sections.

Theorem 3.15. The operator $G_{R}: C^{2}(X) \times C^{1}\left(\mathbb{R}^{m}\right) \rightarrow C(X)$ is Fréchet differentiable and its derivative with respect to the parameterization $z$ is given by

$$
\frac{\partial}{\partial z} G_{R}(z, \zeta) h=\left[G_{R}^{\prime}(z) h+i\left(\zeta G_{D}^{\prime}(z) h+(h \cdot \operatorname{grad} \zeta) u\right)\right] \circ z
$$

and the derivative with respect to the impedance $\zeta$ is given by

$$
\frac{\partial}{\partial \zeta} G_{R}(z, \zeta) \mu=i(\mu u) \circ z
$$


Proof. The Fréchet differentiability of $G_{R}$ is again a consequence of the analyticity of $u$, the $C^{1}$-smoothness of $\zeta$ and the $C^{2}$-smoothness of $z$. To characterize the derivative with respect to the parameterization $z$ we proceed as in the proofs of the Theorems 3.6, 3.11 or 3.13. Using the decomposition (3.54) we get

$$
\begin{aligned}
G_{R}(z+h, \zeta)-G_{R}(z, \zeta)= & G_{N}(z+h)-G_{N}(z)+ \\
& +i\left(\zeta \circ(z+h)\left(G_{D}(z+h)-G_{D}(z)\right)\right. \\
& \left.+G_{D}(z)(\zeta \circ(z+h)-\zeta \circ(z))\right)
\end{aligned}
$$

and using Taylor's formula to get

$$
\zeta((z+h)(x))=\zeta(z(x))+\operatorname{grad} \zeta(z(x)) \cdot h(x)+O\left(\|h\|_{C^{2}}^{2}\right), \quad x \in X
$$

and that

$$
\begin{array}{ll}
G_{N}(z+h)-G_{N}(z)=G_{N}^{\prime}(z)+O\left(\|h\|_{C^{2}}^{2}\right), & x \in X \\
G_{D}(z+h)-G_{D}(z)=G_{D}^{\prime}(z)+O\left(\|h\|_{C^{2}}^{2}\right), & x \in X
\end{array}
$$

the result for the Fréchet derivative of $G_{R}$ with respect to the parameterization follows. As the operator $G_{R}$ is linear in $\zeta$, the Fréchet derivative of $G_{R}$ with respect to the impedance is clearly given by (3.57).

Remark 3.16. To prove the previous result we needed to assume that $\zeta$ is $C^{1}$, while we only have continuity assumptions for the impedance $\lambda$. However we will see later in the beginning of section 5.1.4 that for star-shaped domains the method can be applied in practise to a just continuous impedance, using some proper extension of the function $\zeta_{n}$ from $\gamma_{n}$ to a neighbourhood of it.

With this characterization we solve (3.55) for both $h$ and $\mu$ in a least squares sense and get the new approximations to the boundary $\Gamma$ given by $\gamma_{n+1}$, with parameterization given by $z_{n+1}=z_{n}+h$, and to the impedance $\lambda$ given by $\zeta_{n+1}=\zeta_{n}+\mu$. Details for the numerical implementation in $\mathbb{R}^{2}$ are given in section 5.1.4.

\section{Comparison between the Hybrid Method and a Newton Method}

Now that we have characterized the Fréchet derivative of $G$, we would like to say some words on the comparison between the hybrid method and the usual Newton method applied to the boundary-to-far-field operator $F$ defined in (3.1). Namely, we will compare the characterization of the Fréchet derivative of $G$ to the boundary condition of the forward problem that characterizes the Fréchet derivative of $F$. 
Let again $\Gamma$ (parameterized by $z_{*}$ ) be the solution to the inverse problem. Let $D_{h}$ be a smooth domain with boundary $\Gamma_{h}$, where $\Gamma_{h}$ is a smooth perturbation of $\Gamma$ given by

$$
\Gamma_{h}=\left\{z_{h}(t):=z_{*}(t)+h(t): t \in X\right\} .
$$

We will consider for simplicity that $\bar{D} \subset D_{h}$. We start by noting that the total field $u_{h}$, corresponding to scattering by an obstacle $D_{h}$, satisfies the corresponding boundary condition $B_{\Gamma_{h}} u_{h}=0$ over $\Gamma_{h}$, where $B_{\Gamma_{h}}$ is one of the boundary condition operators (2.8)-(2.10) with $\Gamma$ replaced by $\Gamma_{h}$. The field $u_{h}$ defines the operator $F$, since $F\left(\Gamma_{h}\right)$ is the far-field of the scattered field $u_{h}^{s}$. The characterization of the Fréchet derivative of $F$ is given in terms of a forward problem (see[18, 19, 53]). The procedure of proof to obtain this characterization goes as follows: One wants to characterize a solution $v$ to the Helmholtz equation such that the sum $u_{h}^{s}-u^{s}-v$ goes to zero superlinearly as $h \rightarrow 0$. In this way one would have in particular that

$$
\left.\left(u_{h}^{s}-u^{s}-v\right)\right|_{\gamma_{h}}=o(\|h\|)
$$

as $h \rightarrow 0$. Then, from the far-field pattern representation (2.13) over $\Gamma_{h}$ one concludes that $F^{\prime}(\gamma) h=v_{\infty}$, where $v_{\infty}$ is the far-field pattern of $v$, missing just a characterization of $v$ to complete the proof. The characterization of $v$ is given through the solution to a forward problem with an appropriate boundary condition (see[18, 19, 53]). As we will see in the following lines, this boundary condition is strongly related with the Fréchet derivative of $G$.

We recall the definition of $G$ as the operator that maps a parameterization $z$ to the exterior trace of the boundary condition of a field $u$ over $\gamma$ parameterized by $z$. Considering $u$ to be the exact total field, we define $G: z \mapsto B u \circ z$, where $B$ is again one of the boundary condition operators (2.8)-(2.10). In the same way we define a similar operator $H: z \mapsto B v \circ z$, where $v$ plays the role of $u$. Then formally we obtain

$$
\begin{aligned}
B\left(u_{h}^{s}-u^{s}-v\right) \circ z_{h} & =B(-u-v) \circ z_{h} \\
& =-\left(G\left(z_{h}\right)+H\left(z_{h}\right)\right) \\
& =-\left(G\left(z_{*}\right)+G^{\prime}\left(z_{*}\right) h+H\left(z_{*}\right)+H^{\prime}\left(z_{*}\right) h\right)+O\left(|h|^{2}\right) \\
& =-\left(G^{\prime}\left(z_{*}\right) h+H\left(z_{*}\right)+H^{\prime}\left(z_{*}\right) h\right)+O\left(\|h\|^{2}\right) .
\end{aligned}
$$

Since $v \rightarrow 0$ along with its derivatives as $h \rightarrow 0$, by the characterization of $H^{\prime}$ we have that $H^{\prime}(z) h=o(\|h\|)$. From (3.58) and taking the first order terms in $h$ in the previous equation and by the definition of $H$ we conclude that

$$
(B v) \circ z_{*}=H\left(z_{*}\right)=-G^{\prime}\left(z_{*}\right) h,
$$

that is, the boundary condition that $v$ has to satisfy over $\Gamma$ is equal to $-G^{\prime}\left(z_{*}\right) h$. 
In this way we will characterize the derivative of $G_{R}$ over the correct boundary $\Gamma$ and impedance $\lambda$. The Dirichlet and Neumann cases are particular cases of this procedure. In $\mathbb{R}^{2}$, one can also rewrite (3.56) in the following form

$$
\begin{aligned}
\frac{\partial}{\partial z} G_{R}(z, \zeta) h= & {\left[-k^{2}(h \cdot \nu) u-\frac{\partial}{\partial \tau}\left((h \cdot \nu)\left(\frac{\partial u}{\partial \tau}\right)\right)+(i \zeta+H)(h \cdot \nu) \frac{\partial u}{\partial \nu}\right.} \\
& \left.+(h \cdot \tau)\left(\frac{\partial}{\partial \tau}\left(\frac{\partial u}{\partial \nu}+i \zeta u\right)\right)+i(h \cdot \nu)\left(\frac{\partial \zeta}{\partial \nu} u\right)\right] \circ z
\end{aligned}
$$

since we have the decomposition in the normal and tangential direction

$$
h \cdot(\operatorname{grad} \zeta \circ z)=(h \cdot \tau) \frac{\partial \zeta}{\partial \tau} \circ z+(h \cdot \nu) \frac{\partial \zeta}{\partial \nu} \circ z .
$$

In $\mathbb{R}^{3}$, the characterization (3.56) would take the form

$$
\begin{aligned}
\frac{\partial}{\partial z} G_{R}(z, \zeta) h= & {\left[-k^{2}(h \cdot \nu) u-\operatorname{Div}\left((h \cdot \nu) \nabla_{\tau} u\right)+(i \zeta+2 H)(h \cdot \nu) \frac{\partial u}{\partial \nu}\right.} \\
& \left.+h \cdot\left(\nabla_{\tau}\left(\frac{\partial u}{\partial \nu}+i \zeta u\right)\right)+i(h \cdot \nu)\left(\frac{\partial \zeta}{\partial \nu} u\right)\right] \circ z
\end{aligned}
$$

since we have the decomposition in the normal and tangential components

$$
h \cdot(\operatorname{grad} \zeta \circ z)=\left(h \cdot \nabla_{\tau} \zeta\right) \circ z+(h \cdot \nu) \frac{\partial \zeta}{\partial \nu} \circ z .
$$

Note that if we consider $u$ to be the true total field and if we evaluate the previous Fréchet derivatives on the parameterization $z_{*}$ to the true boundary solution $\Gamma$ and on the true impedance $\lambda$, then the boundary condition is satisfied, that is,

$$
\left(\frac{\partial u}{\partial \nu}+i \lambda u\right) \circ z_{*}=0
$$

and consequently the tangential component of the gradient of the boundary condition would also vanish. Therefore, in $\mathbb{R}^{2}$ the derivative is given by

$$
\begin{aligned}
\frac{\partial}{\partial z} G_{R}\left(z_{*}, \lambda\right) h= & {\left[-k^{2}(h \cdot \nu) u-\frac{\partial}{\partial \tau}\left((h \cdot \nu)\left(\frac{\partial u}{\partial \tau}\right)\right)+(i \lambda+H)(h \cdot \nu) \frac{\partial u}{\partial \nu}\right.} \\
& \left.+i(h \cdot \nu)\left(\frac{\partial \lambda}{\partial \nu} u\right)\right] \circ z_{*}
\end{aligned}
$$


while in $\mathbb{R}^{3}$ it is given by

$$
\begin{aligned}
\frac{\partial}{\partial z} G_{R}\left(z_{*}, \lambda\right) h= & {\left[-k^{2}(h \cdot \nu) u-\operatorname{Div}\left((h \cdot \nu) \nabla_{\tau} u\right)+(i \lambda+2 H)(h \cdot \nu) \frac{\partial u}{\partial \nu}\right.} \\
& \left.+i(h \cdot \nu)\left(\frac{\partial \lambda}{\partial \nu} u\right)\right] \circ z_{*}
\end{aligned}
$$

According to [19], where the work in [18] is extended to a non-constant impedance, this is up to a factor -1 the impedance boundary condition to the boundary value problem that characterizes the Fréchet derivative of the operator $F$ defined in (3.1). In other words and as expected, this is up to a factor -1 the boundary values of the extra forward problem that needs to be solved at each step of the Newton's method applied to the operator defined in (3.1). Heuristically one could say that the factor -1 comes from the fact that $G$ and $F$ depend on $\gamma$ in a symmetric way. While for $G$ the field $u$ is built in a way to satisfy the far-field equation but not the boundary condition on $\gamma$, for $F$ the field $u_{h}$ is constructed in order to satisfy the boundary condition over $\Gamma_{h}$ but not to have the given far-field pattern $u_{\infty}$. In this way we exposed the connections between these methods. 


\section{Chapter 4}

\section{Convergence Results}

In this chapter, we will present some theoretical results on the convergence of the hybrid method. We will study two different approaches to prove convergence for two closely related methods, each one with some drawbacks and gaps when compared to the actual implementation of the hybrid method itself. First, in section 4.1, we start by relating the hybrid method with a minimization problem in a decomposition method spirit. We will follow the ideas of $[9$, sec. 5.4] and extend it to the impedance case. Afterwards, in section 4.2, we will compare the hybrid method to a related iterative Newton method and prove convergence for it, using some ideas of [55]. Both these approaches present still open problems when compared to the practical application of the hybrid method. A reference to these open problems is made at the end of each section.

\subsection{A Related Minimization Problem}

Along the lines of $[9$, sec. 5.4], we will now introduce a minimization problem as theoretical background for the hybrid method. We will establish existence and convergence results for this minimization problem, which is related with the hybrid method as explained later on in this section. In [9] the analysis is done for the Dirichlet case with a single layer representation of the solution, covering the Dirichlet case as presented in section 3.3.

Remark 4.1. For the approach for a sound-soft crack as referred to in remark 3.7 we refer again to [42].

Following the lines of [59], in this section we will apply similar ideas to get convergence of the minimization problem to the Robin case as presented in section 3.5. This can be easily adapted to the particular case of impedance $\lambda=0$, that is, the Neumann case as presented in section 3.4. There are two main differences between what is done in this section and the result in [9, sec. 5.4]. The first is that more regularity is assumed on the density $\varphi$ due to the combined single-and double-layer approach in order to use 
compactness results. The second difference is that here we are concerned with recovering not just the shape of the scatterer as in [9] but also the impedance.

As in chapter 5 we will be only interested in reconstructing star-shaped domains, we will stick to that restriction, that is,

$$
\gamma_{r}=\left\{z(t)=r(\hat{x}(t)) \hat{x}(t) \mid r: \Omega_{m} \rightarrow \mathbb{R}^{m}, t \in X\right\} .
$$

where $\hat{x}(t)$ is defined in $\mathbb{R}^{m}$ by

$$
\hat{x}(t)= \begin{cases}(\cos t, \sin t), & t \in X=[0,2 \pi], m=2, \\ \left(\sin t_{1} \cos t_{2}, \sin t_{1} \sin t_{2}, \cos t_{1}\right), & t \in X=[0, \pi] \times[0,2 \pi], m=3 .\end{cases}
$$

We consider only radial parameterizations $r \in U$, where

$$
U=\left\{r \in H^{l}\left(\Omega_{m}\right) \mid 0<r_{i} \leq\|r\|_{H^{l}\left(\Omega_{m}\right)} \leq r_{e}\right\}
$$

for some fixed $r_{i}, r_{e} \in \mathbb{R}^{+}$. As $\Omega_{m}$ has dimension $(m-1)$ and we want $C^{2}$ domains in $\mathbb{R}^{m}$, in view of the Sobolev imbedding we will assume later on that $l>(m+3) / 2$. We will also consider impedances $\zeta \in V$ where

$$
V=\left\{\zeta: \gamma \rightarrow \mathbb{R} \mid \zeta \in H^{p}(\gamma), 0 \leq \zeta(x) \leq \zeta_{e}, x \in \gamma\right\}
$$

for some fixed $\zeta_{e} \in \mathbb{R}^{+}$and again in order to have continuous impedances $\zeta$ on $\gamma_{r}$, we will assume that $p>(m-1) / 2$. For the density, we will consider $\varphi \in H^{q}(\gamma)$.

Assuming $q>(m+1) / 2+\alpha$, for some fixed $\alpha>0$, we can restrict the operators $F_{\gamma, \infty}$ and $B_{\gamma, \zeta}$ introduced in section 3.5 as operators mapping from $H^{q}(\gamma)$ to $L^{2}\left(\Omega_{m}\right)$ and $L^{2}(\gamma)$, respectively, since by the Sobolev's imbedding theorems $H^{q}(\gamma) \subset C^{1, \alpha}(\gamma)$.

We define the cost function $\Lambda(r, \zeta, \varphi ;):. U \times V \times H^{q}(\gamma) \rightarrow \mathbb{R}_{0}^{+}$given by

$$
\Lambda(r, \zeta, \varphi ; \beta)=\beta\|\varphi\|_{H^{q}\left(\gamma_{r}\right)}^{2}+\Lambda_{1}(r, \zeta, \varphi)+\Lambda_{2}(r, \zeta, \varphi)
$$

for $\beta>0$, where

$$
\Lambda_{1}(r, \zeta, \varphi)=\left\|F_{\gamma_{r}, \infty} \varphi-f\right\|_{L^{2}(\Omega)}^{2}
$$

for some $f \in L^{2}(\Omega)$ and

$$
\Lambda_{2}(r, \zeta, \varphi)=\left\|B_{\gamma_{r}, \zeta} \varphi+g\right\|_{L^{2}\left(\gamma_{r}\right)}^{2}
$$

for some $g \in L^{2}\left(\mathbb{R}^{2}\right)$, where $\gamma_{r}$ is the contour corresponding to $r$.

On the one hand, if $f=u_{\infty}$ and if $r$ and $\zeta$ are fixed, the minimization of

$$
\beta\|\varphi\|_{H^{q}\left(\gamma_{r}\right)}^{2}+\Lambda_{1}(r, \zeta, \varphi)
$$

with respect to $\varphi$ is equivalent to finding a regularized solution to the Tikhonov equation (3.51) with regularization parameter $\beta$, or in other words, to solve the first step of 
each iteration of the hybrid method. On the other hand, if $g$ is the impedance boundary data and $\varphi$ is kept fixed, minimizing $\Lambda_{2}(r, \zeta, \varphi)$ in terms of $r$ and $\zeta$ can be seen as finding a solution to (3.7), or in other words, to solve the second step of each iteration of the hybrid method. In this way this nonlinear optimization problem is related with the hybrid method, joining the two steps in one single cost function. Therefore we will proceed with convergence results for this method as the parameter $\beta \rightarrow 0$.

The first issue is however to guarantee an existence of a solution for every given parameter $\beta$. Given $f$ and $g$, we define the pair $\left(r_{0}, \zeta_{0}\right) \in U \times V$ as being optimal if there exists $\varphi_{0} \in H^{q}\left(\gamma_{0}\right)$ such that

$$
\Lambda\left(r_{0}, \zeta_{0}, \varphi_{0} ; \beta\right)=M(\beta)
$$

where

$$
M(\beta):=\inf _{r \in U, \zeta \in V, \varphi \in H^{q}(\gamma)} \Lambda(r, \zeta, \varphi ; \beta) .
$$

We can show the following existence theorem.

Theorem 4.2. Assume in addition to $q>(m+1) / 2+\alpha$ that also $l>(m+3) / 2$ and $p>(m-1) / 2$. Then for each $\beta>0, f \in L^{2}(\Omega)$ and $g \in L^{2}\left(\mathbb{R}^{m}\right)$ there exists an optimal pair $(r, \zeta) \in U \times V$.

Proof. Assume the triple $\left(r_{n}, \zeta_{n}, \varphi_{n}\right)$ to be a minimizing sequence, that is,

$$
\lim _{n \rightarrow \infty} \Lambda\left(r_{n}, \zeta_{n}, \varphi_{n} ; \beta\right)=M(\beta) .
$$

As $U$ is bounded in $H^{l}\left(\Omega_{m}\right)$, by the Sobolev compact embedding theorems one has that $H^{l}\left(\Omega_{m}\right) \subset C^{2}\left(\Omega_{m}\right)$ and so $U$ is compact in $C^{2}\left(\Omega_{m}\right)$. Therefore, without loss of generality, we can assume $C^{2}$-convergence $r_{n} \rightarrow r$ as $n \rightarrow \infty$. As $\mathrm{U}$ is closed, $r \in U$. In a similar way one can assume $C$-convergence of $\zeta_{n} \rightarrow \zeta \in V$.

One also has that

$$
\beta\left\|\varphi_{n}\right\|_{H^{q}\left(\gamma_{r}\right)}^{2} \leq \Lambda\left(r_{n}, \zeta_{n}, \varphi_{n} ; \beta\right) \rightarrow M(\beta)
$$

as $n \rightarrow \infty$. So $\varphi_{n}$ is bounded and by a similar argument using the compact embedding $H^{l}(X) \subset C^{1, \alpha}(X)$ one can assume that $\varphi_{n} \rightarrow \varphi$. By continuity of the functional $\Lambda$ in all its variables, one has the result, since

$$
\Lambda(r, \zeta, \varphi ; \beta)=\lim _{n \rightarrow \infty} \Lambda\left(r_{n}, \zeta_{n}, \varphi_{n} ; \beta\right)=M(\beta) .
$$

We are interested in the behaviour of a solution to the minimization problem as the regularization parameter $\beta$ goes to zero. One can state the following convergence result. Note that, unfortunately, it does not imply convergence to a solution to the inverse scattering problem, since there is no general result to prove uniqueness for the inverse problem with one incident wave. 
Theorem 4.3. Assume $q>(m+1) / 2+\alpha, l>(m+3) / 2$ and $p>(m-1) / 2$. Assume also that $f$ is the exact far-field pattern $u_{\infty}$, that $g$ is the exact boundary data given by $g=\partial u^{i} / \partial \nu+i \lambda u^{i}$, that the solution $\Gamma$ can be parameterized by some $r_{*} \in U$ and that the exact impedance $\lambda \in V$. Let $\left(\beta_{n}\right)$ be a null sequence and let $\left(r_{n}, \zeta_{n}\right)$ be a sequence of corresponding optimal pairs. Then there exists a convergent subsequence of $\left(r_{n}, \zeta_{n}\right)$ and every limit point $\left(r^{\dagger}, \zeta^{\dagger}\right)$ represents a curve $\gamma^{\dagger}$ and impedance $\zeta^{\dagger}$ such that

$$
\frac{\partial u^{s}}{\partial \nu}+i \zeta^{\dagger} u^{s}=-g \text { on } \gamma^{\dagger}
$$

Proof. From section 3.5, one knows that the solution to the direct problem can be represented by a combined single and double layer potential via the solution $\varphi$ to the equation $B_{\Gamma, \lambda} \varphi=g$. Therefore with the assumptions on $f$ and $g$

$$
\Lambda_{1}\left(r_{*}, \lambda, \varphi\right)=\Lambda_{2}\left(r_{*}, \lambda, \varphi\right)=0
$$

Then, by the assumptions that $r_{*} \in U$ and that $\lambda \in V$ one has that

$$
\lim _{\beta \rightarrow 0} M(\beta)=0 .
$$

since

$$
M(\beta) \leq \Lambda\left(r_{*}, \lambda, \varphi ; \beta\right)=\beta\|\varphi\|_{H^{q}\left(\gamma_{r}\right)}^{2} .
$$

The existence of a convergent subsequence $\left(r_{k(n)}, \zeta_{k(n)}\right)_{n \in \mathbb{N}}$ comes from the proof of theorem 4.2. For simplicity we will denote $k=k(n)$. Let $\left(r^{\dagger}, \zeta^{\dagger}\right)$ be the limit point of that convergent subsequence and let $u^{\dagger}$ be the solution to the direct scattering problem with boundary condition

$$
\frac{\partial u^{\dagger}}{\partial \nu}+i \zeta^{\dagger} u^{\dagger}=-g \quad \text { on } \gamma^{\dagger}
$$

Since $\left(r_{k}, \zeta_{k}\right)$ is optimal there exists $\left(\varphi_{k}\right)_{k \in \mathbb{N}}$ such that

$$
\Lambda\left(r_{k}, \zeta_{k}, \varphi_{k}, \beta_{k}\right)=M\left(\beta_{k}\right) .
$$

Let now $u_{k}$ be the combined single and double layer potential over $\gamma_{k}$ (with radial parameterization $r_{k}$ ) with density $\varphi_{k}$. The potential $u_{k}$ can be interpreted as the solution to the exterior scattering problem with boundary $\gamma_{k}$ and impedance $\zeta_{k}$.

From (4.3), we observe that

$$
\left\|\frac{\partial u_{k}}{\partial \nu}+i \zeta_{k} u_{k}+g\right\|_{L^{2}\left(\gamma_{k}\right)}^{2} \leq M\left(\beta_{k}\right) \rightarrow 0
$$

and

$$
\left\|F_{\gamma_{k}, \infty} \varphi_{k}-f\right\|_{L^{2}(\Omega)}^{2} \leq M\left(\beta_{k}\right) \rightarrow 0
$$


both as $k \rightarrow \infty$.

By (4.4) one concludes that $u_{k}$ and all its derivatives converge to $u^{\dagger}$ on compact sets of the exterior domain (e.g. thm. 5.16 in [9]) and so the far-field patterns of $u_{k}$ also converge to the far-field pattern $u_{\infty}^{\dagger}$ of $u^{\dagger}$. By (4.5) we conclude that $u_{\infty}^{\dagger}=f=u_{\infty}$ and so $u^{s}=u^{\dagger}$ follows. This concludes the proof.

This approach can be carried out for all the boundary conditions, but has some drawbacks when compared with the actual implementation of the hybrid method. As already referred, this approach joins both steps of a decomposition method in one minimization problem. In this way there is a gap between the theoretical argument and the practical application of the hybrid method, since in practise one first minimizes the $\operatorname{sum} \beta\|\varphi\|_{H^{q}\left(\gamma_{r}\right)}^{2}+\Lambda_{1}(r, \zeta, \varphi)$ with respect to $\varphi$ and then minimizes $\Lambda_{2}(r, \zeta, \varphi)$ with respect to $r$. It is not clear whether this minimization in two steps used in the hybrid method leads to the same result as the minimization of $\Lambda(r, \zeta, \varphi)$ simultaneously in $r, \zeta$ and $\varphi$ that was treated theoretically in this section. Moreover, this approach does not take into account the linearization in the second step of the hybrid method, which does not exactly correspond to minimizing the cost functional $\Lambda_{2}$. The iterative procedure of the hybrid method is also not taken into account, nor is any noise level that might be added in the given far-field.

In the next section we will present a different approach that deals with these drawbacks. However, it can only be applied to the Dirichlet case. As in this approach, it has also the drawback that the penalty term for Tikhonov regularization is in a Sobolev norm $\left(H^{q}\right.$-norm), while in practice a $L^{2}$-norm penalty term is used and leads to successful reconstructions. This is confirmed by [34] where significant influence of the norm on the penalty term was not found in the behaviour of the solution using the Kirsch and Kress method.

\subsection{A Related Newton's Method}

In this section we will establish a local convergence result for the hybrid method. We will follow the same procedure for a convergence proof as in [55], with differences in the considered operators. In [55] the convergence analysis is done for the boundary-to-farfield pattern operator (3.1), or in other words, for the usual Newton method applied to the operator that maps the boundary to the far-field pattern of the corresponding scattered wave. In this work we are interested in a different operator that is related to the hybrid method. We will define the hybrid method as a pointwise iterative scheme and derive its convergence under some assumptions. The analysis will be performed just for the Dirichlet case. The extension to the Neumann and Robin cases does not seem to be possible in a general sense, as addressed at the end of this section. Again we assume the domain to be star-shaped, that is, the boundary is of the form (4.1), but a proof can be 
carried out for a general-shaped and sufficiently smooth domain.

\section{Noise free data}

For a first analysis, we assume that the given far-field data $u_{\infty}$ is noise free.

Considering star-shaped domains as in (4.1), let $r_{n}$ be the radial parameterization for the current approximation $\gamma_{n}$ and let $r_{*}$ be the radial parameterization for the solution $\Gamma$ to the inverse problem. The first step of the hybrid method consists of reconstructing the scattered field. To do so, we assume that the solution $u_{*}$ to the forward problem can be analytically extended (possibly through the interior of $D$ ) up to $\gamma_{n}$, as referred in the analytic continuation principle 3.2. Then it is possible to represent $u^{s}$ as a combined layer potential over $\gamma_{n}$

$$
u^{s}(x)=\int_{\gamma_{n}}\left(\frac{\partial \Phi(x, y)}{\partial \nu(y)}-i \eta \Phi(x, y)\right) \varphi_{*}^{(n)}(y) d s(y), \quad x \in \mathbb{R}^{m} \backslash \gamma_{n}
$$

with density $\varphi_{*}^{(n)}$ being the unique solution to the far-field equation

$$
F_{\gamma_{n}, \infty} \varphi_{*}^{(n)}=u_{\infty}
$$

where again $F_{\gamma, \infty}=\left(K_{\gamma, \infty}-i \eta S_{\gamma, \infty}\right)$.

Remark 4.4. We just used a combined single-and double-layer representation to get rid of the assumption that $k^{2}$ is not an interior eigenvalue of the successive approximations $\gamma_{n}$. With this last assumption, a single-layer representation could be carried out in exactly the same way.

Therefore, at each step $n$ the reconstructed total field represented as a combined layer potential over $\gamma_{n}$ coincides with the true solution $u_{*}$ to the forward problem, as well as its trace and normal trace on the boundary, respectively, given by

$$
u_{*}=u^{i}+L_{\gamma_{n}} \varphi_{*}^{(n)} \quad \text { on } \gamma_{n}
$$

and

$$
\frac{\partial u_{*}}{\partial \nu}=\frac{\partial u^{i}}{\partial \nu}+N_{\gamma_{n}} \varphi_{*}^{(n)} \quad \text { on } \gamma_{n}
$$

where the operators involved are defined as

$$
\begin{aligned}
& L_{\gamma}=\left(I / 2+K_{\gamma}-i \eta S_{\gamma}\right), \\
& N_{\gamma}=\left(i \eta I / 2+T_{\gamma}-i \eta K_{\gamma}^{*}\right) .
\end{aligned}
$$

Using the hybrid method for the Dirichlet case, we end up at each step with the linearized equation

$$
\left.\left(u_{*}+\operatorname{grad} u_{*} \cdot h\right)\right|_{\gamma_{n}}=0
$$


that for star-shaped domains with some abuse of notation $h(\hat{x})=h \hat{x}$ reduces to

$$
\left.\left(u_{*}+\frac{\partial u_{*}}{\partial \hat{x}} h\right)\right|_{\gamma_{n}}=0
$$

We now assume that the radial derivatives do not vanish in a small closed neighbourhood $U$ of $\Gamma$, that is,

$$
\left|\frac{\partial u_{*}}{\partial \hat{x}}\right|_{\gamma} \mid>0
$$

for $\gamma \in U$. For continuous radial derivatives the condition (4.9) implies there exists an $\epsilon>0$ such that

$$
\left|\frac{\partial u_{*}}{\partial \hat{x}}\right|_{\gamma} \mid \geq 4 \epsilon
$$

for $\gamma \in U$.

Remark 4.5. One needs to justify that the previous assumption is not artificial and makes sense in practise. In [2, pp.360], it is shown that for scattering by a sound-soft sphere of radius $a$ in $\mathbb{R}^{3}$ with plane wave incidence with direction $d=(0,0,-1)$, the normal derivative of the corresponding total field $u$ measured over the same sphere is given explicitly by

$$
\frac{1}{k} \frac{\partial u}{\partial \hat{x}}(a \hat{x}(\theta, \phi))=\frac{1}{k} \frac{\partial u}{\partial \nu}(a \hat{x}(\theta, \phi))=-\frac{i}{(k a)^{2}} \sum_{n=0}^{\infty}(-i)^{n}(2 n+1) \frac{P_{n}(\cos \theta)}{h_{n}^{(1)}(k a)}
$$

for $\hat{x}(\theta, \phi)$ given as in (4.2) and where $h_{n}^{(1)}$ holds for a spherical Bessel function of the first kind and order $n$ (e.g. [9, sec.2.4]). Plots of $|1 / k \partial u / \partial \hat{x}|$ (see [2, fig.10.2] or figure 4.1) for several values of $k a$ show numerically that the radial derivative on the surface does not vanish. Therefore, by continuous dependence of the total field $u$ on the scatterer, we conclude that for domains close to a sphere it makes sense to make the assumption (4.9). However, the value of $|1 / k \partial u / \partial \hat{x}|$ gets smaller in the shadow region as $k a$ increases, so assumption (4.10) might not hold for large obstacles or high frequency.

Then, the linearized equation can be written pointwise as

$$
h=-\left.\left(u_{*} / \frac{\partial u_{*}}{\partial \hat{x}}\right)\right|_{\gamma_{n}} .
$$

Note that the quantity on the left-hand side of the previous equation is real-valued, while the quantity on the right-hand side might be complex-value. Therefore, the shift might be computed for numerical purposes by

$$
h=-\left.\operatorname{Re}\left(u_{*} / \frac{\partial u_{*}}{\partial \hat{x}}\right)\right|_{\gamma_{n}}
$$




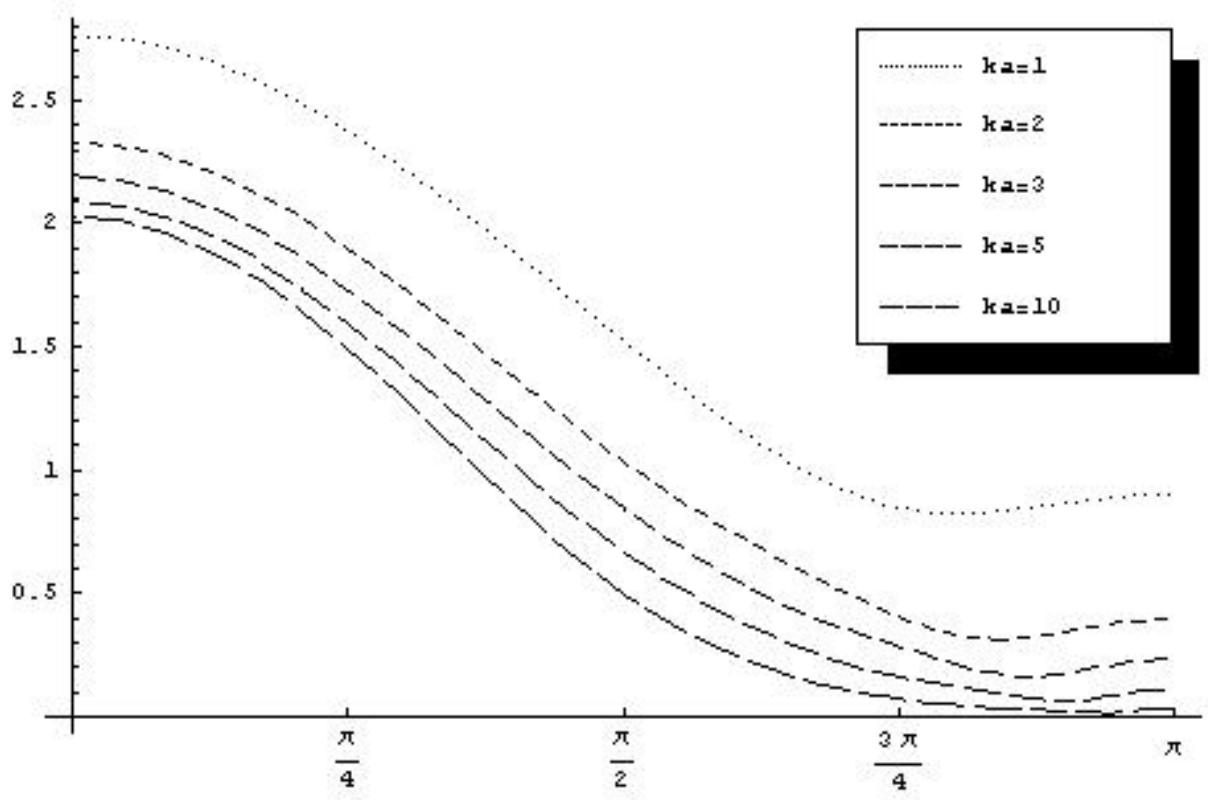

Figure 4.1: Plot of $|1 / k \partial u / \partial \hat{x}|$ in terms of $\theta$.

In this case we have that (4.10) must be replaced by

$$
\left|\operatorname{Re}\left(\left.\frac{\partial u_{*}}{\partial \hat{x}}\right|_{\gamma}\right)\right|>4 \epsilon
$$

which is still covered by a similar argument as in remark 4.5 for low frequency.

Remark 4.6. Another possibility is to consider

$$
h=-\left.\frac{1}{2}\left(\operatorname{Re}\left(u_{*} / \frac{\partial u_{*}}{\partial \hat{x}}\right)+\operatorname{Im}\left(u_{*} / \frac{\partial u_{*}}{\partial \hat{x}}\right)\right)\right|_{\gamma_{n}} .
$$

This is a more natural way since one fits both the real and imaginary parts when trying to find the zeros. In this case we would have that (4.10) must be replaced by

$$
\left|\operatorname{Re}\left(\left.\frac{\partial u_{*}}{\partial \hat{x}}\right|_{\gamma}\right)\right|>4 \epsilon, \quad\left|\operatorname{Im}\left(\left.\frac{\partial u_{*}}{\partial \hat{x}}\right|_{\gamma}\right)\right|>4 \epsilon .
$$

In order to keep the notation shorter we will continue the analysis for (4.12). One should have in mind that all the estimates obtained also hold for this last case (4.14) just by using the property that the $C$-norm of a complex function is greater than or equal to the $C$-norm of its real or imaginary part.

Therefore the update is obtained by the iterative scheme

$$
r_{n+1}=r_{n}-\left.\operatorname{Re}\left(u_{*} / \frac{\partial u_{*}}{\partial \hat{x}}\right)\right|_{\gamma_{n}},
$$


where we recall that $u_{*}$ is the true solution to the forward problem with scatterer $D$. Note that given an analytic initial guess $r_{0}$, at each step the updated approximation $r_{n+1}$ is still analytic, due to the analyticity of $u_{*}$.

By Taylor's Formula

$$
\left|u_{*}\left(r_{n}(\hat{x}) \hat{x}\right)-u_{*}\left(r_{*}(\hat{x}) \hat{x}\right)-\frac{\partial u_{*}\left(r_{n}(\hat{x}) \hat{x}\right)}{\partial \hat{x}}\left(r_{n}(\hat{x})-r_{*}(\hat{x})\right)\right|=O\left(\left|r_{n}(\hat{x})-r_{*}(\hat{x})\right|^{2}\right)
$$

and by the boundary condition, as $\left.u_{*}\right|_{\Gamma}=0$, we get

$$
\left\|\left.u_{*}\right|_{\gamma_{n}}-\left.\frac{\partial u_{*}}{\partial \hat{x}}\right|_{\gamma_{n}}\left(r_{n}-r_{*}\right)\right\|_{\infty}=O\left(\left\|r_{n}-r_{*}\right\|^{2}\right)
$$

In this way we obtain

$$
\begin{aligned}
r_{n+1}-r_{*} & =r_{n}-\left.\operatorname{Re}\left(u_{*} / \frac{\partial u_{*}}{\partial \hat{x}}\right)\right|_{\gamma_{n}}-r_{*} \\
& =\operatorname{Re}\left[\left(\left.\left(r_{n}-r_{*}\right) \frac{\partial u_{*}}{\partial \hat{x}}\right|_{\gamma_{n}}-\left.u_{*}\right|_{\gamma_{n}}\right) /\left(\left.\frac{\partial u_{*}}{\partial \hat{x}}\right|_{\gamma_{n}}\right)\right] .
\end{aligned}
$$

which implies that

$$
\left\|r_{n+1}-r_{*}\right\| \leq \frac{C}{4 \epsilon}\left\|r_{n}-r_{*}\right\|^{2}
$$

showing that (4.16) converges (superlinearly) to the solution in a small neighbourhood $U$ of $\Gamma$.

\section{Noisy data}

If the far-field data $u_{\infty}^{\delta} \in L^{2}\left(\Omega_{m}\right)$ has noise with magnitude $\delta$, that is,

$$
\left\|u_{\infty}^{\delta}-u_{\infty}\right\|_{L^{2}\left(\Omega_{m}\right)}<\delta
$$

then (4.6) in general has no solution. Therefore we look for a regularized solution to (4.6), that is,

$$
\varphi_{\alpha}^{(n)}:=R_{n, \alpha} u_{\infty}^{\delta}
$$

where $R_{n, \alpha}$ is a regularization scheme for $F_{\gamma_{n}, \infty}^{-1}$. As before we apply Tikhonov regularization, that is,

$$
R_{n, \alpha}=\left(\alpha I+F_{\gamma_{n}, \infty}^{*} F_{\gamma_{n}, \infty}\right)^{-1} F_{\gamma_{n}, \infty}^{*} .
$$

Therefore the iterative scheme (4.16) must be replaced by

$$
r_{n+1}=r_{n}-\left.\operatorname{Re}\left(u_{n, \alpha} / \frac{\partial u_{n, \alpha}}{\partial \hat{x}}\right)\right|_{\gamma_{n}}
$$


where

$$
\begin{array}{r}
\left.u_{n, \alpha}\right|_{\gamma_{n}}=\left.u^{i}\right|_{\gamma_{n}}+L_{\gamma_{n}} R_{n, \alpha} u_{\infty}^{\delta} \\
\left.\frac{\partial u_{n, \alpha}}{\partial \hat{x}}\right|_{\gamma_{n}}=\left.\frac{\partial u^{i}}{\partial \hat{x}}\right|_{\gamma_{n}}+D_{\gamma_{n}} R_{n, \alpha} u_{\infty}^{\delta} .
\end{array}
$$

and

$$
D_{\gamma}:\left.\varphi \mapsto \hat{x} \cdot\left(\nu N_{\gamma} \varphi+\nabla_{\tau} L_{\gamma} \varphi\right)\right|_{\gamma}
$$

maps $\varphi_{\alpha}^{(n)}$ into $\left.\left(\frac{\partial u_{n, \alpha}^{s}}{\partial \hat{x}}\right)\right|_{\gamma_{n}}$. In $\mathbb{R}^{m}$, we consider the operators

$$
\begin{gathered}
F_{\gamma, \infty}: H^{m}(\gamma) \rightarrow L^{2}\left(\Omega_{m}\right) \\
L_{\gamma}: H^{m}(\gamma) \rightarrow H^{m}(\gamma) \\
N_{\gamma}: H^{m}(\gamma) \rightarrow H^{m-1}(\gamma), \\
D_{\gamma}: H^{m}(\gamma) \rightarrow H^{m-1}(\gamma),
\end{gathered}
$$

that by Sobolev's imbedding theorems and theorem 2.11 are bounded for a $C^{4, \alpha}$-smooth surface $\gamma$ and in particular for analytic boundaries.

By theorem 3.10, in $\mathbb{R}^{m}$ it makes sense to consider the regularization operator

$$
R_{n, \alpha}: L^{2}(\gamma) \rightarrow H^{m}(\gamma)
$$

as defined in (4.19). In this way, it is clear that the exterior trace $\left.u_{n, \alpha}\right|_{\gamma_{n}} \in H^{m}\left(\gamma_{n}\right)$ and also that the exterior normal trace $\left.\left(\partial u_{n, \alpha} / \partial \hat{x}\right)\right|_{\gamma_{n}} \in H^{m-1}\left(\gamma_{n}\right)$. Therefore we can consider (4.27) in a pointwise sense, since for a contour $\gamma \in \mathbb{R}^{m}$ by the Sobolev imbedding theorems the $H^{m-1}(\gamma)$-norm over $\gamma$ is stronger than the $C(\gamma)$-norm. However, unlike in the no noise case, by (4.20) even if $r_{n}$ is analytic the update $r_{n+1}$ is just in $H^{m-1}\left(\Omega_{m}\right)$. In this way, one needs extra regularization so that the operators $(4.23)-(4.26)$ remain bounded in the considered spaces for the next Newton step. Having this in mind, we redefine the iterative method as

$$
\tilde{r}_{n+1}=r_{n}-\left.\operatorname{Re}\left(u_{n, \alpha} / \frac{\partial u_{n, \alpha}}{\partial \hat{x}}\right)\right|_{\gamma_{n}}
$$

and $r_{n+1}=Q\left(\tilde{r}_{n+1}\right)$, where $Q: C\left(\Omega_{m}\right) \rightarrow C^{4, \alpha}\left(\Omega_{m}\right)$ maps $\tilde{r}_{n+1}$ to a quasi-solution $r_{n+1}$ with constraint $C_{0}$, that is,

$$
\left\|\tilde{r}_{n+1}-r_{n+1}\right\|_{C\left(\Omega_{m}\right)} \leq\left\|\tilde{r}_{n+1}-r\right\|_{C\left(\Omega_{m}\right)}
$$

for all $r \in C^{4, \alpha}\left(\Omega_{m}\right)$ with $\|r\|_{C^{4, \alpha}\left(\Omega_{m}\right)} \leq C_{0}$. We will assume as a priori knowledge that $\left\|r_{*}\right\|_{C\left(\Omega_{m}\right)} \leq C_{0}$. 
Remark 4.7. In practise, one does not follow the procedure of finding a quasi-solution at each step. Instead, one finds the best approximation to $\tilde{r}_{n+1}$ in some sufficiently smooth finite dimensional approximation space.

We are now in a position to head for the convergence result. We start by establishing the estimates

$$
\begin{aligned}
\left\|u_{n, \alpha}-u_{*}\right\|_{C\left(\gamma_{n}\right)} & =\left\|L_{\gamma_{n}} \varphi_{\alpha}^{(n)}-L_{\gamma_{n}} \varphi_{*}^{(n)}\right\|_{C\left(\gamma_{n}\right)} \\
& \leq\left\|L_{\gamma_{n}}\right\|\left\|\varphi_{\alpha}^{(n)}-\varphi_{*}^{(n)}\right\|_{C\left(\gamma_{n}\right)} \\
& \leq\left\|L_{\gamma_{n}}\right\|\left\|R_{n, \alpha}\left(u_{\infty}^{\delta}-u_{\infty}\right)+R_{n, \alpha} L_{\gamma_{n}}^{\infty} \varphi_{*}^{(n)}-\varphi_{*}^{(n)}\right\|_{C\left(\gamma_{n}\right)} \\
& \leq C\left(\left\|R_{n, \alpha}\right\| \delta+\left\|R_{n, \alpha} L_{\gamma_{n}}^{\infty} \varphi_{*}^{(n)}-\varphi_{*}^{(n)}\right\|_{C\left(\gamma_{n}\right)}\right) .
\end{aligned}
$$

For Tikhonov regularization, picking $\alpha(\delta) \rightarrow 0$ such that

$$
\frac{\delta^{2}}{\alpha(\delta)} \rightarrow 0, \text { for } \delta \rightarrow 0
$$

we have that (see Thm.4.13 in [9])

$$
\left\|u_{n, \alpha}-u_{*}\right\|_{C\left(\gamma_{n}\right)} \leq \xi_{1}(\delta)
$$

where $\xi_{1}(\delta)$ is monotonously decreasing and $\xi_{1}(\delta) \rightarrow 0$ as $\delta \rightarrow 0$. By a similar argument we can conclude that

$$
\begin{aligned}
\left\|\frac{\partial u_{n, \alpha}}{\partial \hat{x}}-\frac{\partial u_{*}}{\partial \hat{x}}\right\|_{C\left(\gamma_{n}\right)} & \leq\left\|D_{\gamma_{n}} \varphi_{\alpha}^{(n)}-D_{\gamma_{n}} \varphi_{*}^{(n)}\right\|_{C\left(\gamma_{n}\right)} \\
& \leq \tilde{C}\left(\left\|R_{n, \alpha}\right\| \delta+\left\|R_{n, \alpha} L_{\gamma_{n}}^{\infty} \varphi_{*}^{(n)}-\varphi_{*}^{(n)}\right\|_{C\left(\gamma_{n}\right)}\right)
\end{aligned}
$$

and again similarly we conclude that

$$
\left\|\frac{\partial u_{n, \alpha}}{\partial \hat{x}}-\frac{\partial u_{*}}{\partial \hat{x}}\right\|_{C\left(\gamma_{n}\right)} \leq \xi_{2}(\delta)
$$

such that $\xi_{2}(\delta)$ is monotonously decreasing and $\xi_{2}(\delta) \rightarrow 0$ as $\delta \rightarrow 0$. Note that if (4.10) holds, then for a sufficiently small closed neighbourhood $U$ of $\Gamma$ and a sufficiently small $\delta$ we have

$$
\left|\frac{\partial u_{n, \alpha}}{\partial \hat{x}}\right|_{\gamma} \mid \geq 2 \epsilon
$$

for $\gamma \in U$.

As the method involves regularization, a stopping rule is of major importance to establish convergence of the method. 
Definition 4.8 (Stopping Rule). Given noisy data $u_{\infty}^{\delta}$ fulfilling (4.17) we stop the iterative scheme (4.27) if two successive approximations satisfy

$$
\left\|r_{n+1}-r_{n}\right\|_{C\left(\Omega_{m}\right)} \leq C_{1}(\delta)
$$

where $C_{1}(\delta)=\frac{4 \xi_{1}(\delta)}{\epsilon-2 \xi_{2}(\delta)}$.

Note that the stopping rule gets more strict as $\delta$ decreases, since $C_{1}(\delta) \rightarrow 0$ as $\delta \rightarrow 0$. When the stopping criteria is fulfilled, we establish by definition $r_{\delta}:=r_{n+1}$.

Theorem 4.9 (Convergence). Let $\Gamma$ be analytic and assume that (4.30) holds. Then the iterative scheme (4.27) with a regularization scheme satisfying (4.28) and (4.29) with a stopping rule (4.31) is locally convergent, in the sense that

$$
\left\|r_{\delta}-r_{*}\right\|_{C\left(\Omega_{m}\right)} \rightarrow 0, \quad \delta \rightarrow 0 .
$$

Proof. As compared to the noise free data, the proof must be changed in the following way. We start by noting that by definition of a quasi-solution, since the solution $r_{*}$ is assumed to be analytic (and therefore $r_{*} \in C^{4, \alpha}(\Omega)$ ) we have

$$
\begin{aligned}
\left\|r_{n+1}-r_{*}\right\|_{C\left(\Omega_{m}\right)} & \leq\left\|r_{n+1}-\tilde{r}_{n+1}\right\|_{C\left(\Omega_{m}\right)}+\left\|\tilde{r}_{n+1}-r_{*}\right\|_{C\left(\Omega_{m}\right)} \\
& \leq 2\left\|\tilde{r}_{n+1}-r_{*}\right\|_{C\left(\Omega_{m}\right)} .
\end{aligned}
$$

We also have that

$$
\begin{aligned}
\tilde{r}_{n+1}-r_{*}= & r_{n}-\left.\operatorname{Re}\left(u_{n, \alpha} / \frac{\partial u_{n, \alpha}}{\partial \hat{x}}\right)\right|_{\gamma_{n}}-r_{*} \\
= & \operatorname{Re}\left[\left(-\left.u_{n, \alpha}\right|_{\gamma_{n}}+\left.\left(r_{n}-r_{*}\right) \frac{\partial u_{n, \alpha}}{\partial \hat{x}}\right|_{\gamma_{n}}\right) /\left(\left.\frac{\partial u_{n, \alpha}}{\partial \hat{x}}\right|_{\gamma_{n}}\right)\right] \\
= & \operatorname{Re}\left[\left\{-\left.u_{*}\right|_{\gamma_{n}}-\left.\left(r_{*}-r_{n}\right) \frac{\partial u_{*}}{\partial \hat{x}}\right|_{\gamma_{n}}+\left.\left(u_{*}-u_{n, \alpha}\right)\right|_{\gamma_{n}}\right.\right. \\
& \left.\left.+\left.\left(r_{n}-r_{*}\right)\left(\frac{\partial u_{n, \alpha}}{\partial \hat{x}}-\frac{\partial u_{*}}{\partial \hat{x}}\right)\right|_{\gamma_{n}}\right\} /\left(\left.\frac{\partial u_{n, \alpha}}{\partial \hat{x}}\right|_{\gamma_{n}}\right)\right]
\end{aligned}
$$

and therefore by Taylor's formula and by (4.28) and (4.29) we get

$$
\begin{aligned}
\left\|r_{n+1}-r_{*}\right\|_{C\left(\Omega_{m}\right)} & \leq 2\left\|\tilde{r}_{n+1}-r_{*}\right\|_{C\left(\Omega_{m}\right)} \\
& \leq \frac{1}{\epsilon}\left(C\left\|r_{n}-r_{*}\right\|_{C\left(\Omega_{m}\right)}^{2}+\xi_{2}(\delta)\left\|r_{n}-r_{*}\right\|_{C\left(\Omega_{m}\right)}+\xi_{1}(\delta)\right)
\end{aligned}
$$

where $C$ is a constant depending on the solution $u_{*}$. 
In order to estimate the error on the $(n+1)$-approximation in terms of the error on the $n$-approximation, we want to explore under which conditions we have

$$
\frac{1}{\epsilon}\left(C\left\|r_{n}-r_{*}\right\|_{C\left(\Omega_{m}\right)}^{2}+\xi_{2}(\delta)\left\|r_{n}-r_{*}\right\|_{C\left(\Omega_{m}\right)}+\xi_{1}(\delta)\right)<\frac{\left\|r_{n}-r_{*}\right\|_{C\left(\Omega_{m}\right)}}{2} .
$$

Defining $\beta:=\epsilon / 2-\xi_{2}(\delta)$, that is positive for a sufficiently small $\delta$, by the solution formula for quadratic equations we have

$$
\frac{\beta-\sqrt{\beta^{2}-4 C \xi_{1}(\delta)}}{2 C}<\left\|r_{n}-r_{*}\right\|_{C\left(\Omega_{m}\right)}<\frac{\beta+\sqrt{\beta^{2}-4 C \xi_{1}(\delta)}}{2 C} .
$$

Therefore, by Taylor's expansion of the square root function around $\beta$, for a sufficiently small fixed $\delta$ one concludes that if

$$
C_{1}(\delta)=\frac{4 \xi_{1}(\delta)}{\epsilon-2 \xi_{2}(\delta)}<\left\|r_{n}-r_{*}\right\|_{C\left(\Omega_{m}\right)}<\frac{\epsilon-2 \xi_{2}(\delta)}{4 C}
$$

we get

$$
\left\|r_{n+1}-r_{*}\right\|_{C\left(\Omega_{m}\right)} \leq \frac{\left\|r_{n}-r_{*}\right\|_{C\left(\Omega_{m}\right)}}{2} .
$$

This shows that for a sufficiently small $\delta$, we have that (4.33) holds for a starting value in some neighbourhood of $\Gamma$, as long as $r_{n}$ satisfies (4.32). In order to justify the choice for the stopping criteria, we note that under (4.32) we have that

$$
\begin{aligned}
\left\|r_{n+1}-r_{n}\right\|_{C\left(\Omega_{m}\right)} & =\left\|r_{n+1}-r_{*}-\left(r_{n}-r_{*}\right)\right\|_{C\left(\Omega_{m}\right)} \\
& \geq\left\|r_{n}-r_{*}\right\|_{C\left(\Omega_{m}\right)}-\left\|r_{n+1}-r_{*}\right\|_{C\left(\Omega_{m}\right)} \\
& \geq \frac{1}{2}\left\|r_{n}-r_{*}\right\|_{C\left(\Omega_{m}\right)}
\end{aligned}
$$

and therefore if the stopping criteria (4.31) is satisfied then $r_{n}$ would be the last iteration to satisfy (4.32) since

$$
\left\|r_{n+1}-r_{*}\right\|_{C\left(\Omega_{m}\right)} \leq \frac{\left\|r_{n}-r_{*}\right\|_{C\left(\Omega_{m}\right)}}{2} \leq\left\|r_{n+1}-r_{n}\right\|_{C\left(\Omega_{m}\right)} \leq C_{1}(\delta)
$$

and further convergence could not be guaranteed. Therefore, by definition of $r_{\delta}$ one gets

$$
\left\|r_{\delta}-r_{*}\right\|_{C\left(\Omega_{m}\right)} \leq C_{1}(\delta) \rightarrow 0
$$

as $\delta \rightarrow 0$ and the proof is finished.

This result proves convergence of the hybrid method for the Dirichlet case under certain assumptions, taking into account the iterative procedure and the linearization of the second step, unlike the minimization problem approach in section 4.1. However, 
some of the assumptions need further work. Assumption (4.9) still requires a rigorous theoretical proof and its validity can be only supported in some cases, as for domains close to spheres in the low frequency case (see remark 4.5). This approach also requires more smoothness on the boundary, requiring analyticity (or at least $C^{4, \alpha}$-smooth, which is a very artificial space). Another drawback of this approach is that its extension to the Neumann (and consequently to the Robin) case is not trivial, since the characterization of $G_{N}^{\prime}$ depends on $h$ and its tangential derivatives. Therefore we are not able to write the shift $h$ explicitly (as in (4.11) for the Dirichlet case) for a general $h$ and therefore we do not get an iterative method of the form (4.16) equivalent to the hybrid method. 


\section{Chapter 5}

\section{Numerical Results}

In this chapter we present the numerical procedure to implement the hybrid method as well as the numerical results obtained. The main difficulty lies in the numerical evaluation of the integral operators involved, namely the layer potentials, so we based our approach on previous work on quadrature rules to deal with their singularities. In this way we split our presentation in the two-and three-dimensional cases, since the approach is different for each case. For the two-dimensional case there exist exponentially convergent quadrature rules obtained by explicitly integrating the singularities involved based on trigonometric interpolation (see [9, sec. 3.5.] and [36]). These quadrature rules lead to numerically solving the integral equations by the Nyström method. For the threedimensional case, such a procedure is not convenient. We will base ourselves in $[12,15]$, where a Galerkin method is presented to solve the direct problem dealing with the previously mentioned singularities of the fundamental solution. Convergence rates where proven for this Galerkin method (see [12]), namely super-algebraic convergence for analytic right-hand sides. Then we will use some of its ideas to establish quadrature rules to numerically integrate the integral operators appearing in the inverse problem. These quadrature rules end up to be the same as introduced by Wienert [65], that are also presented in an english version in $\left[9\right.$, sec.3.6]. In $\mathbb{R}^{2}$ we also split the treatment for each considered boundary condition (2.8)-(2.10), with special attention to the Robin case, where both the scatterer $D$ and the impedance $\lambda$ are reconstructed. In $\mathbb{R}^{3}$ we just present the numerical implementation of the method and the corresponding reconstructions for the Dirichlet case due to simplicity, though an extension to other boundary conditions is possible with similar changes as in the two-dimensional problem. Numerical results show the feasibility of the method, being its robustness also displayed in reconstructions from noisy data. 


\subsection{Two-dimensional case}

In this section we will present the numerical procedure to solve the inverse problem 2.15 by the hybrid method in $\mathbb{R}^{2}$. For simplicity, in this section we will denote the unit sphere by $\Omega:=\Omega_{2}$. We will briefly present the direct problem in order to introduce the quadrature rule to deal with the logarithmic singularities of the fundamental solution. In this way we present how the direct problem was solved in order to generate the synthetic far-field data for the inverse problem. Then, in the following three sections 5.1.2 -5.1.4 we will present the numerical procedure to solve the inverse problem for the Dirichlet, Neumann and Robin boundary condition, respectively.

\subsubsection{Direct problem}

We will start by briefly explaining how we obtained the synthetical data, solving the direct problem. The goal is to compute the far-field pattern $u_{\infty}$ corresponding to scattering by a given obstacle $D$ with boundary $\Gamma$ with a given incident field $u^{i}$. We represented the scattered field as a combined single-and double-layer potential over $\Gamma$

$$
u^{s}(x)=\int_{\Gamma}\left(\frac{\partial \Phi(x, y)}{\partial \nu(y)}-i \eta \Phi(x, y)\right) \varphi(y) d s(y), \quad x \in \mathbb{R}^{m} \backslash \Gamma
$$

with $\eta>0$, which is possible to do requiring enough regularity on $\Gamma$ and on the incident field $u^{i}$ over the boundary (see [8]). For each of the boundary conditions (2.8)-(2.10), the scattered field $u^{s}$ must satisfy

$$
B u^{s}=-B u^{i} \quad \text { on } \Gamma .
$$

Considering the layer operators given in section 2.2.1, one is lead to the following integral equation for the Dirichlet boundary condition (2.8)

$$
\frac{\varphi}{2}+(K-i \eta S) \varphi=-u^{i} \quad \text { on } \Gamma,
$$

making use of the jump relations. For the Neumann boundary condition (2.9) we get

$$
i \eta \frac{\varphi}{2}+\left(T-i \eta K^{*}\right) \varphi=-\frac{\partial u^{i}}{\partial \nu} \quad \text { on } \Gamma .
$$

As already referred in (3.49), for the Robin boundary condition (2.10) we get

$$
B_{\Gamma, \lambda} \varphi=-\left(\frac{\partial u^{i}}{\partial \nu}+i \lambda u^{i}\right) \quad \text { on } \Gamma
$$

where the boundary operator is given by

$$
B_{\gamma, \zeta}=i(\eta+\zeta) \frac{I}{2}+T_{\gamma}+i\left(\zeta K_{\gamma}-\eta K_{\gamma}^{*}\right)+\zeta \eta S_{\gamma}
$$


Considering $\varphi$ in the appropriate smooth space, all the previous equations are integral equations of the second kind (e.g. [9]). Assuming to have the boundary $\Gamma$ in a parametric form, that is,

$$
\Gamma=\{z(t): t \in[0,2 \pi]\}
$$

where $z$ is a $C^{2}$-smooth $2 \pi$-periodic and counter-clockwise oriented parameterization, the next step is to parameterize the previous integral equations. Then, by the Nyström method, one just needs to straightforwardly approximate the integrals by appropriate quadrature rules, and collocate the equation in the quadrature points in order to obtain a linear system.

For the operators $S, K$ and $K^{*}$ we simply use the quadrature rules in [9, sec. 3.5.], which are exponentially convergent for analytic boundaries $\Gamma$.

First we consider the Dirichlet case, that is, we write (5.1) in the parametric form

$$
\psi(s)+2 \int_{0}^{2 \pi} M(s, t) \psi(t) d t=2 g(s), \quad s \in[0,2 \pi],
$$

where $\psi(s)=\varphi(z(s))$ and $g(s)=-u^{i}(z(s))$ and

$$
M(s, t)=M^{K}(s, t)-i \eta M^{S}(s, t), \quad s, t \in[0,2 \pi],
$$

where $M^{S}$ and $M^{K}$ are respectively the parametric kernels of the single-and double-layer operators that will be defined in a few lines. The goal is to decompose the parameterized kernel $M$ in the form

$$
M(s, t)=M_{1}(s, t) \ln \left(4 \sin ^{2} \frac{s-t}{2}\right)+M_{2}(s, t), \quad s \neq t
$$

for $s, t \in[0,2 \pi]$, where $M_{1}$ and $M_{2}$ are analytic. This can be done by expanding the fundamental solution $H_{0}^{(1)}=J_{0}+i N_{0}$ in its power series (e.g. [9]). The idea is to apply this decomposition to the parametric kernel $M^{S}$ of the single layer operator $S$ and to the parametric kernel $M^{K}$ of the double layer operator $K$ and then make use of (5.5) to obtain the decomposition (5.6).

Therefore, following the ideas of $[44,49]$ described in $[9$, sec.3.5], we have that the parametric kernel $M^{S}$ of the single layer operator $S$, such that

$$
(S \varphi)(z(s))=\int_{0}^{2 \pi} M^{S}(s, t) \psi(t) d t, \quad s \in[0,2 \pi]
$$

can be decomposed as

$$
\begin{aligned}
M^{S}(s, t) & :=\frac{i}{4} H_{0}^{(1)}(k|z(s)-z(t)|)\left|z^{\prime}(t)\right| \\
& =M_{1}^{S}(s, t) \ln \left(4 \sin ^{2} \frac{s-t}{2}\right)+M_{2}^{S}(s, t)
\end{aligned}
$$


where

$$
\begin{aligned}
& M_{1}^{S}(s, t):=-\frac{1}{4 \pi} J_{0}(k|z(s)-z(t)|)\left|z^{\prime}(t)\right| \\
& M_{2}^{S}(s, t):=M^{S}(s, t)-M_{1}^{S}(s, t) \ln \left(4 \sin ^{2} \frac{s-t}{2}\right)
\end{aligned}
$$

are analytic and $M_{2}^{S}$ has a diagonal term given by

$$
M_{2}^{S}(t, t)=\left(\frac{i}{4}-\frac{C}{2 \pi}-\frac{1}{4 \pi} \ln \left(\frac{k^{2}}{4}\left|z^{\prime}(t)\right|^{2}\right)\right)\left|z^{\prime}(t)\right|
$$

where $C=0.5772156649 \ldots$ is Euler's constant.

In a similar way the parametric kernel $M^{K}$ of the double-layer operator $K$ can be decomposed as

$$
\begin{aligned}
M^{K}(s, t) & :=\frac{i k}{4} \frac{[(z(s)-z(t)) \cdot \nu(z(t))]}{|z(s)-z(t)|} H_{1}^{(1)}(k|z(s)-z(t)|)\left|z^{\prime}(t)\right| \\
& =M_{1}^{K}(s, t) \ln \left(4 \sin ^{2} \frac{s-t}{2}\right)+M_{2}^{K}(s, t)
\end{aligned}
$$

where

$$
\begin{aligned}
& M_{1}^{K}(s, t):=-\frac{k}{4 \pi} \frac{[(z(s)-z(t)) \cdot \nu(z(t))]}{|z(s)-z(t)|} J_{1}(k|z(s)-z(t)|)\left|z^{\prime}(t)\right| \\
& M_{2}^{K}(s, t):=M^{K}(s, t)-M_{1}^{K}(s, t) \ln \left(4 \sin ^{2} \frac{s-t}{2}\right)
\end{aligned}
$$

are also analytic with diagonal term

$$
M_{2}^{K}(t, t)=\frac{1}{4 \pi} \frac{z^{\prime \prime}(t) \cdot \nu(z(t))}{\left|z^{\prime}(t)\right|}
$$

Note that though $M^{K}$ is continuous, this decomposition brings advantages since its derivatives are not continuous at $s=t$. We also note that $H_{1}^{(1)}=-H_{0}^{(1)^{\prime}}$ denotes the Hankel function of first kind and order one and $J_{1}=-J_{0}^{\prime}$ denotes the Bessel function of order one. For $K^{*}$ everything follows in a very similar way (see [36]), getting

$$
\begin{aligned}
M^{K^{*}}(s, t) & :=-\frac{i k}{4} \frac{[(z(s)-z(t)) \cdot \nu(z(s))]}{|z(s)-z(t)|} H_{1}^{(1)}(k|z(s)-z(t)|)\left|z^{\prime}(t)\right| \\
& =M_{1}^{K^{*}}(s, t) \ln \left(4 \sin ^{2} \frac{s-t}{2}\right)+M_{2}^{K^{*}}(s, t)
\end{aligned}
$$


where

$$
\begin{aligned}
& M_{1}^{K^{*}}(s, t):=\frac{k}{4 \pi} \frac{[(z(s)-z(t)) \cdot \nu(z(s))]}{|z(s)-z(t)|} J_{1}(k|z(s)-z(t)|)\left|z^{\prime}(t)\right| \\
& M_{2}^{K^{*}}(s, t):=M^{K}(s, t)-M_{1}^{K^{*}}(s, t) \ln \left(4 \sin ^{2} \frac{s-t}{2}\right)
\end{aligned}
$$

are also analytic with diagonal term

$$
M_{2}^{K^{*}}(t, t)=\frac{1}{4 \pi} \frac{z^{\prime \prime}(t) \cdot \nu(z(t))}{\left|z^{\prime}(t)\right|} .
$$

In this way the logarithmic singularity of these kernels is exposed explicitly.

Having equation (5.4) in mind, one can proceed using the quadrature rule for the equidistant points $t_{j}:=\pi j / N, j=0, \ldots, 2 N-1$ given by

$$
\int_{0}^{2 \pi} \ln \left(4 \sin ^{2} \frac{s-t}{2}\right) f(t) d t \approx \sum_{j=0}^{2 N-1} R_{j}^{(N)}(s) f\left(t_{j}\right)
$$

with weights

$$
R_{j}^{(N)}(s):=-\frac{2 \pi}{N} \sum_{l=1}^{N-1} \frac{1}{l} \cos l\left(s-t_{j}\right)-\frac{\pi}{N^{2}} \cos N\left(s-t_{j}\right)
$$

and the trapezoidal rule

$$
\int_{0}^{2 \pi} f(t) d t \approx \frac{\pi}{N} \sum_{j=0}^{2 N-1} f\left(t_{j}\right)
$$

both obtained by replacing the integrand $f$ by its trigonometric interpolation polynomial and then integrating exactly. We end up with an approximated equation of the form

$$
\psi(s)+2 \sum_{j=0}^{2 N-1}\left(R_{j}^{(N)}(s) M_{1}\left(s, t_{j}\right)+\frac{\pi}{N} M_{2}\left(s, t_{j}\right)\right) \psi\left(t_{j}\right)=2 g(s), \quad s \in[0,2 \pi] .
$$

In particular, by the Nyström method, for $\psi_{i}=\psi\left(t_{i}\right), i=0, \ldots, 2 N-1$ we get the linear system

$$
\psi_{i}+2 \sum_{j=0}^{2 N-1}\left(R_{|i-j|}^{(N)} M_{1}\left(t_{i}, t_{j}\right)+\frac{\pi}{N} M_{2}\left(t_{i}, t_{j}\right)\right) \psi_{j}=2 g\left(t_{i}\right), \quad i=0, \ldots, 2 N-1
$$

where the quadrature weights can be simplified to the form

$$
R_{j}^{(N)}=-\frac{2 \pi}{N} \sum_{l=1}^{N-1} \frac{1}{l} \cos \frac{l j \pi}{N}-\frac{(-1)^{N} \pi}{N^{2}} .
$$


In this way one obtains a reconstruction of the density $\psi$ that can be used to obtain the far-field $u_{\infty}$ from the integral far-field representation of the combined single-and doublelayer potential. We will refer to that at the end of this section, after showing how to reconstruct the density for the Neumann and Robin cases.

As for equations (5.2) and (5.3), corresponding to the Neumann and Robin cases respectively, one still needs to introduce a parameterized version of the integral operator $T$. However, as we will see in a few lines, a parametric integral equation of the form (5.4) will not be achieved. Using the identity (see [50])

$$
T \varphi=\frac{d}{d \tau} S \frac{d \varphi}{d \tau}+k^{2} \nu \cdot S(\nu \varphi) .
$$

and following [36] one can show that

$$
\left(\frac{d}{d \tau} S \frac{d \varphi}{d \tau}\right)(z(s))=\frac{1}{\left|z^{\prime}(s)\right|} \int_{0}^{2 \pi}\left(\frac{1}{4 \pi} \cot \frac{t-s}{2} \frac{d \varphi\left(z^{\prime}(t)\right)}{d \tau}-N(s, t) \varphi(z(t))\right) d t
$$

where

$$
\begin{aligned}
N(s, t):= & \frac{i}{4} \tilde{N}(s, t)\left[k^{2} H_{0}^{(1)}(k|z(s)-z(t)|)-\frac{2 k H_{1}^{(1)}(k|z(s)-z(t)|)}{|z(s)-z(t)|}\right] \\
& +\frac{i k z^{\prime}(s) \cdot z^{\prime}(t)}{4|z(s)-z(t)|} H_{1}^{(1)}(k|z(s)-z(t)|)+\frac{1}{8 \pi} \frac{1}{\sin ^{2} \frac{s-t}{2}}
\end{aligned}
$$

with

$$
\tilde{N}(s, t):=\frac{z^{\prime}(s) \cdot(z(s)-z(t)) z^{\prime}(t) \cdot(z(s)-z(t))}{|z(s)-z(t)|^{2}} .
$$

Moreover, we have the decomposition

$$
N(s, t)=N_{1}(s, t) \ln \left(4 \sin ^{2} \frac{s-t}{2}\right)+N_{2}(s, t)
$$

where

$$
\begin{aligned}
N_{1}(s, t):= & -\frac{1}{4 \pi} \tilde{N}(s, t)\left[k^{2} J_{0}(k|z(s)-z(t)|)-\frac{2 k H_{1}^{(1)}(k|z(s)-z(t)|)}{|z(s)-z(t)|}\right] \\
& -\frac{k z^{\prime}(s) \cdot z^{\prime}(t)}{4 \pi|z(s)-z(t)|} J_{1}(k|z(s)-z(t)|) \\
N_{2}(s, t):= & N(s, t)-N_{1}(s, t) \ln \left(4 \sin ^{2} \frac{s-t}{2}\right)
\end{aligned}
$$


are analytic with diagonal terms

$$
\begin{aligned}
N_{1}(t, t)= & -\frac{k^{2}\left|z^{\prime}(t)\right|^{2}}{8 \pi}, \\
N_{2}(t, t)= & \left(\pi i-1-2 C-2 \ln \frac{k\left|z^{\prime}(t)\right|}{2}\right) \frac{k^{2}\left|z^{\prime}(t)\right|}{8 \pi} \\
& +\frac{1}{24 \pi}+\frac{\left[z^{\prime}(t) \cdot z^{\prime \prime}(t)\right]^{2}}{4 \pi\left|z^{\prime}(t)\right|^{4}}-\frac{\left|z^{\prime \prime}(t)\right|^{2}}{8 \pi\left|z^{\prime}(t)\right|^{2}}-\frac{z^{\prime}(t) \cdot z^{\prime \prime \prime}(t)}{12 \pi\left|z^{\prime}(t)\right|^{2}} .
\end{aligned}
$$

Using the identity

$$
\nu(z(s)) \cdot \nu(z(t))=\frac{z^{\prime}(s) \cdot z^{\prime}(t)}{\left|z^{\prime}(s)\right|\left|z^{\prime}(t)\right|}
$$

we also get

$$
\left(k^{2} \nu \cdot S(\nu \varphi)\right)(z(s))=\frac{k^{2}}{\left|z^{\prime}(s)\right|} \int_{0}^{2 \pi}\left(z^{\prime}(s) \cdot z^{\prime}(t)\right) \frac{M^{S}(s, t)}{\left|z^{\prime}(t)\right|} \psi(t) d t
$$

Therefore, one can write $T$ in the parametric form

$$
(T \varphi)(z(s))=\frac{1}{\left|z^{\prime}(s)\right|}\left(\frac{1}{4 \pi} \int_{0}^{2 \pi} \cot \frac{t-s}{2} \psi^{\prime}(t) d t+\int_{0}^{2 \pi} M^{T}(s, t) \psi(t) d t\right)
$$

where

$$
M^{T}(s, t):=k^{2}\left(z^{\prime}(s) \cdot z^{\prime}(t)\right) \frac{M^{S}(s, t)}{\left|z^{\prime}(t)\right|}-N(s, t) .
$$

In this way, the integral equations (5.2) and (5.3) can be written in the parametric form as

$$
\psi(s)+\frac{1}{\xi}\left(\frac{1}{\left|z^{\prime}(s)\right|} \frac{1}{4 \pi} \int_{0}^{2 \pi} \cot \frac{t-s}{2} \psi^{\prime}(t) d t+\int_{0}^{2 \pi} M(s, t) \psi(t) d t\right)=\frac{1}{\xi} g(s),
$$

for $s \in[0,2 \pi]$, where again $\psi(s)=\varphi(z(s))$ and both the constant $\xi>0$ and the function $g$ depend on which of the boundary conditions one considers and are given by

$$
\left\{\begin{array}{lll}
\xi=i \eta / 2, & g(s)=-\frac{\partial u}{\partial \nu}(z(s)), & \text { for Neumann } \\
\xi=i(\eta+\lambda) / 2, & g(s)=-\frac{\partial u}{\partial \nu}(z(s))-i \lambda(z(s)) u(z(s)), & \text { for Robin. }
\end{array}\right.
$$

For the Neumann case (5.2) the kernel $M$ appearing in (5.18) is given by

$$
M(s, t)=\frac{1}{\left|z^{\prime}(s)\right|} M^{T}(s, t)-i \eta M^{K^{*}}
$$


while for the Robin case (5.3) the kernel $M$ is given by

$$
M(s, t)=\frac{1}{\left|z^{\prime}(s)\right|} M^{T}(s, t)+i\left(\lambda M^{K}-\eta M^{K^{*}}\right)+\lambda \eta M^{S} .
$$

Replacing the integrand by its trigonometric interpolation polynomial and integrating exactly one gets the quadrature rule given by

$$
\frac{1}{4 \pi} \int_{0}^{2 \pi} \cot \frac{t-s}{2} f^{\prime}(t) d t \approx \sum_{j=0}^{2 N-1} T_{j}^{(N)}(s) f\left(t_{j}\right)
$$

with weights

$$
T_{j}^{(N)}(s)=-\frac{1}{2 N} \sum_{l=1}^{N-1} l \cos l\left(s-t_{j}\right)-\frac{1}{2} \cos N\left(s-t_{j}\right) .
$$

Therefore, treating the second integral on the left-hand side of (5.18) as in the Dirichlet case with the quadrature scheme (5.13) and the trapezoidal rule (5.14), as a consequence of applying the Nyström method to solve (5.18), one ends up with a linear system of the form

$$
\psi_{i}+\frac{1}{\xi} \sum_{j=0}^{2 N-1}\left(\frac{1}{\left|z^{\prime}\left(t_{i}\right)\right|} T_{|i-j|}^{(N)}+R_{|i-j|}^{(N)} M_{1}\left(t_{i}, t_{j}\right)+\frac{\pi}{N} M_{2}\left(t_{i}, t_{j}\right)\right) \psi_{j}=\frac{1}{\xi} g\left(t_{i}\right),
$$

for $i=0, \ldots, 2 N-1$, where the quadrature weights can be simplified to the form

$$
T_{j}^{(N)}= \begin{cases}-\frac{N}{4}, & j=0 \\ \frac{1}{4 N \sin \left(t_{j} / 2\right)}, & j \text { odd } \\ 0, & j \text { even }\end{cases}
$$

Solving the linear system (5.15) or (5.19) according to the boundary condition, we will get the $\psi_{i}, i=0, \ldots, 2 N-1$ corresponding to $\psi$ evaluated at $t_{i}$. As the far-field pattern of a combined single-and double-layer potential is given by

$$
u_{\infty}(\hat{x})=\left(\left(K_{\infty}-i \eta S_{\infty}\right) \varphi\right)(x), \quad \hat{x} \in \Omega
$$

and both $S_{\infty}$ and $K_{\infty}$ given by (2.22) and (2.23), respectively, have a continuous kernel, we simply use the trapezoidal rule (5.14) to compute the far-field pattern by

$$
u_{\infty}(\hat{x}) \approx \frac{\pi}{N} \sum_{j=0}^{2 N-1} M_{\infty}\left(\hat{x}, t_{j}\right) \psi_{j}, \quad \hat{x} \in \Omega
$$


where $M_{\infty}(\hat{x}, t)$ is the parametric kernel of the combined single-and double-layer farfield operator given by

$$
M_{\infty}(\hat{x}, t)=\frac{e^{-i \pi / 4}}{\sqrt{8 \pi k}}(k \hat{x} \cdot \nu(z(t))+\eta) e^{-i k \hat{x} \cdot z(t)}\left|z^{\prime}(t)\right| .
$$

Using $N=100$, we computed the far-field pattern for one incident direction at 100 equidistant points on the unit circle $\Omega$ and considered it as the given data for the inverse scattering problem. As suggested in [35] we choose $\eta=k$.

\subsubsection{Inverse Problem for the Dirichlet Case}

We are now in a position to present the numerical method for the inverse problem, since all the quadrature rules and its ideas have been already mentioned in the previous paragraphs concerning the direct problem. For the inverse problem that we will now discuss, we consider star-shaped obstacles, that is, the boundary of the obstacle is given by

$$
\gamma_{r}=\left\{z(t)=r(\hat{x}(t)) \hat{x}(t) \mid r: \Omega \rightarrow \mathbb{R}^{2}, t \in[0,2 \pi]\right\}
$$

where $\hat{x}(t)$ is defined in $\mathbb{R}^{2}$ by

$$
\hat{x}(t)=(\cos t, \sin t), \quad t \in X=[0,2 \pi] .
$$

As for parameterization space we choose trigonometric polynomials, since they are dense in $L^{2}[0,2 \pi]$ (see [37]). In this way, we consider radial functions $r$ that are linear combinations of trigonometric polynomials of order less than or equal to $N_{z}$, that is,

$$
r(t)=a_{0}+\sum_{j=1}^{N_{z}} a_{j} \cos j t+\sum_{j=1}^{N_{z}} b_{j} \sin j t
$$

with $a_{j}, b_{j} \in \mathbb{R}, j=1, \ldots, N_{z}$. This assumption will be carried out not only for Dirichlet but also for both the Neumann and Robin cases.

In this section we will present the numerical implementation of the hybrid method for the Dirichlet boundary condition presented in section 3.3. Consider $\gamma_{n}$, parameterized by $z_{n}$, to be the current approximation to the solution $\Gamma$ of the inverse problem.

In a first step, the total-field $u$ is reconstructed from the given far-field data $u_{\infty}$, by representing the scattered field $u^{s}$ by a single-layer potential over $\gamma_{n}$, that is,

$$
u^{s}(x)=\int_{\gamma_{n}} \Phi(x, y) \varphi(y) d y .
$$

This representation leads to less complexity in the implementation than a combined single-and double-layer potential and can be taken under the assumptions discussed in 
section 3.3. In this way, as referred in section 3.3, we have to solve the regularized far-field equation

$$
\left(\alpha_{n} I+S_{\gamma_{n}, \infty}^{*} S_{\gamma_{n}, \infty}\right) \varphi^{(n)}=S_{\gamma_{n}, \infty}^{*} u_{\infty}
$$

where

$$
\left(S_{\gamma, \infty} \varphi\right)(\hat{x})=\frac{e^{i \pi / 4}}{\sqrt{8 \pi k}} \int_{\gamma} e^{-i k \hat{x} \cdot y} \varphi(y) d s(y), \quad \hat{x} \in \Omega .
$$

By the relation (e.g. [64, Ch.53])

$$
\pi J_{0}(|y|)=\int_{0}^{\pi} \cos (|y| \cos t) d t=\frac{1}{2} \int_{\Omega} e^{i \hat{x} \cdot y} d \hat{x}, \quad y \in \mathbb{R}^{2}
$$

one can write (5.22) in the parameterized form

$$
\begin{aligned}
\alpha_{n} \psi^{(n)}(s)+\frac{1}{4 k} \int_{0}^{2 \pi} J_{0}\left(k\left|z_{n}(s)-z_{n}(t)\right|\right)\left|z_{n}^{\prime}(t)\right| \psi^{(n)}(t) d t & = \\
& =\frac{e^{-i \pi / 4}}{\sqrt{8 \pi k}} \int_{0}^{2 \pi} e^{i k \hat{x}(t) \cdot z_{n}(s)} u_{\infty}(\hat{x}(t)) d t
\end{aligned}
$$

where $\psi^{(n)}(t)=\varphi^{(n)}\left(z_{n}(t)\right)$. Since all the integral kernels involved in the previous equation are continuous, by the Nyström method associated with the trapezoidal rule (5.14) one gets the linear system

$$
\begin{aligned}
\alpha_{n} \psi_{i}^{(n)}+\frac{\pi}{4 k N} \sum_{j=0}^{2 N-1} J_{0}\left(k \mid z_{n}\left(t_{i}\right)\right. & \left.-z_{n}\left(t_{j}\right) \mid\right)\left|z_{n}^{\prime}\left(t_{j}\right)\right| \psi_{j}^{(n)}= \\
& =\frac{e^{-i \pi / 4}}{\sqrt{8 N k}} \sum_{j=0}^{2 N-1} e^{i k \hat{x}\left(t_{j}\right) \cdot z_{n}\left(t_{i}\right)} u_{\infty}\left(\hat{x}\left(t_{j}\right)\right)
\end{aligned}
$$

for $i=0, \ldots, 2 N-1$, which is solved in order to obtain the $\psi_{i}^{(n)}=\psi^{(n)}\left(t_{i}\right)$.

By the jump relations for the single layer operator (see thm. 2.9)

$$
\begin{aligned}
u^{s}(x) & =S_{\gamma_{n}} \varphi, \\
\frac{\partial u^{s}}{\partial \nu}(x) & =-\frac{\varphi}{2}+K_{\gamma_{n}}^{*} \varphi
\end{aligned}
$$

following the same procedure mentioned in the forward problem (that is, the quadrature rule (5.13) to deal with the logarithmic singularity of the kernels involved and the 
trapezoidal rule (5.14) to deal with the smooth part), one gets the approximations

$$
\begin{aligned}
u^{s}\left(z_{n}\left(t_{i}\right)\right) & \approx u_{n}^{s}\left(z_{n}\left(t_{i}\right)\right):=\sum_{j=0}^{2 N-1}\left(R_{|i-j|}^{(N)} M_{1}^{S}\left(t_{i}, t_{j}\right)+\frac{\pi}{N} M_{2}^{S}\left(t_{i}, t_{j}\right)\right) \psi_{j}^{(n)}, \\
\frac{\partial u^{s}}{\partial \nu}\left(z_{n}\left(t_{i}\right)\right) & \approx \frac{\partial u_{n}^{s}}{\partial \nu}\left(z_{n}\left(t_{i}\right)\right) \\
& :=-\frac{\psi_{i}^{(n)}}{2}+\sum_{j=0}^{2 N-1}\left(R_{|i-j|}^{(N)} M_{1}^{K^{*}}\left(t_{i}, t_{j}\right)+\frac{\pi}{N} M_{2}^{K^{*}}\left(t_{i}, t_{j}\right)\right) \psi_{j}^{(n)},
\end{aligned}
$$

for $i=0, \ldots, 2 N-1$, where the kernels $M_{1}^{S}, M_{2}^{S}$ and $M_{1}^{K^{*}}, M_{2}^{K^{*}}$ are given respectively by (5.7)-(5.8) and (5.11)-(5.12) and the quadrature weights are given by (5.16). One now computes the tangential derivative of the total field $u$ over $\gamma_{n}$ by trigonometric interpolation, that is, one takes the tangential derivative of the trigonometric interpolation polynomial of $u_{n}$ as an approximation to the tangential derivative of the total field $u$. In this way, one can find an approximation to the gradient of the total field using the decomposition

$$
\left.\left.\operatorname{grad} u\right|_{\gamma_{n}} \approx \nu \frac{\partial u_{n}}{\partial \nu}\right|_{\gamma_{n}}+\left.\tau \frac{\partial u_{n}}{\partial \tau}\right|_{\gamma_{n}}
$$

where $u_{n}=u^{i}+u_{n}^{s}$ and the last term on the right hand side is, as already referred, computed by trigonometric differentiation.

From the linearized equation (3.14) and the approximation space for the approximation given by (5.20) and (5.21) one gets

$$
\left(a_{0}^{(h)}+\sum_{j=1}^{N_{z}} a_{j}^{(h)} \cos j t_{i}+\sum_{j=1}^{N_{z}} b_{j}^{(h)} \sin j t_{i}\right) \operatorname{grad} u_{n}\left(z_{n}\left(t_{i}\right)\right) \cdot\left(\cos t_{i}, \sin t_{i}\right)=-u_{n}\left(z_{n}\left(t_{i}\right)\right)
$$

for $i=0, \ldots, 2 N-1$ and so one now fits the coefficients $a_{j}^{(h)}, b_{j}^{(h)}, j=0, \ldots, N_{z}$ by a Levenberg-Marquardt step (note that one must have $N_{z}<N$ ) in order to establish the shift

$$
h(t)=\left(a_{0}^{(h)}+\sum_{j=1}^{N_{z}} a_{j}^{(h)} \cos j t+\sum_{j=1}^{N_{z}} b_{j}^{(h)} \sin j t\right)(\cos t, \sin t), \quad t \in[0,2 \pi]
$$

and get a new approximation $\gamma_{n+1}$ parameterized by $z_{n+1}=z_{n}+h$. We then repeat the process while $\left\|u_{n}\right\|_{L^{2}\left(\gamma_{n}\right)}$ is decreasing.

For the numerical examples in this section we considered the wave number $k=1$ and an incident plane field $u^{i}(x)=e^{i k x \cdot d}, d \in \Omega$. The incident direction $d$ is represented 
in the figures by a grey arrow. We considered as approximation space for the radial parameterization trigonometric polynomials of order less than or equal to $N_{z}=20$ and as regularization parameter $\alpha_{n}=10^{-8} \times 0.5^{n}$ for Tikhonov regularization at the first step of each iteration $n$ of the method. We also note that the stopping criteria was achieved between 5 and 10 iterations for the presented examples.

We first considered an obstacle with parameterization given by

$$
z(t)=(2+0.3 \cos 3 t)(\cos t, \sin t), \quad t \in[2, \pi]
$$

that lies inside the approximation space.
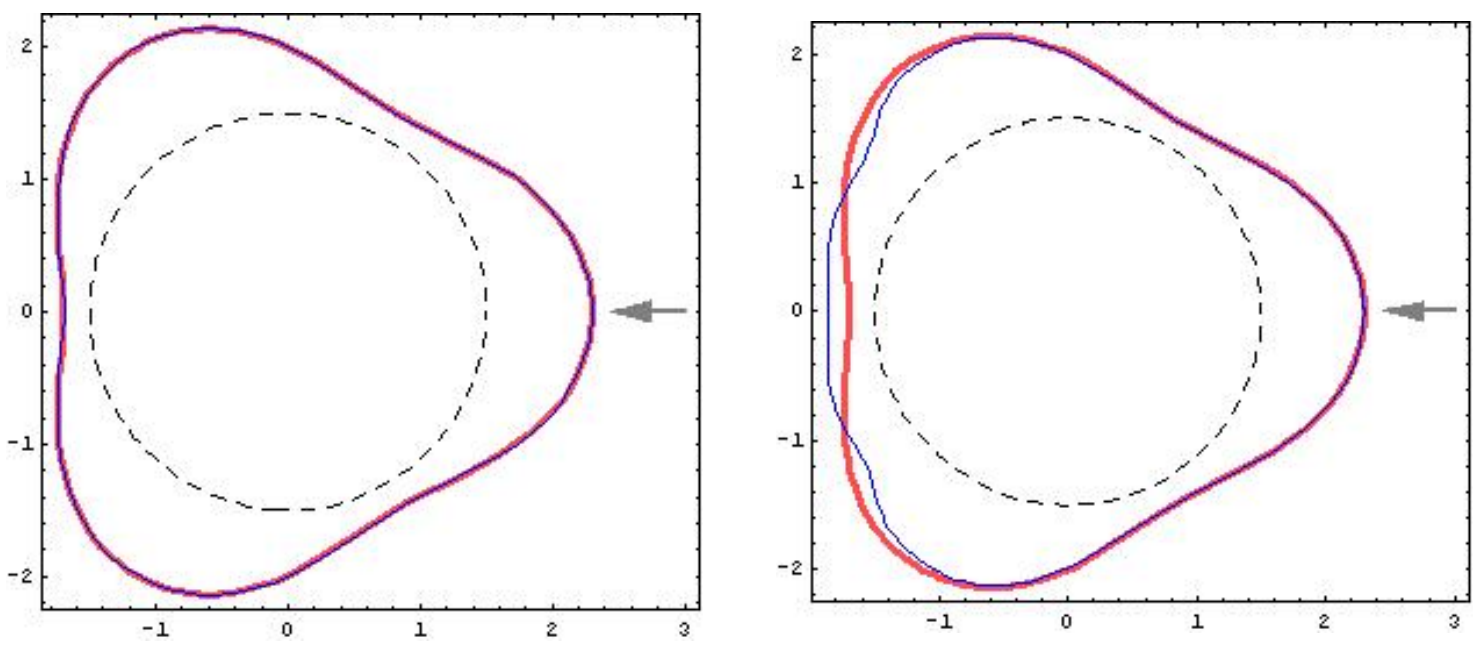

Figure 5.1: Reconstruction (in blue) of a sound-soft obstacle in the approximation space (in red) with exact data (left) and 5\% noise (right). The dashed line is the initial guess.

As one can see in figure 5.1 the reconstruction is perfect considering data without noise. With noisy data the reconstruction deteriorates mostly in the shadow region, as usual for methods using data from just one incident plane wave.

As a second example we considered a peanut shaped obstacle parameterized by

$$
z(t)=\sqrt{\cos ^{2} t+0.25 \sin ^{2} t}(\cos t, \sin t), \quad t \in[2, \pi] .
$$

As shown in figure 5.2, again the reconstructions are quite good. It is clear that the approximation becomes worst in the non-convex region of the peanut, since this part is not illuminated by the considered incident direction. With noisy data the reconstruction deteriorates in the shadow region as in the previous example.

As a third and last example for sound-soft obstacles, we considered a kite-shaped obstacle parameterized by

$$
z(t)=(\cos t+0.65 \cos 2 t-0.65,1.5 \sin t), \quad t \in[2, \pi] .
$$



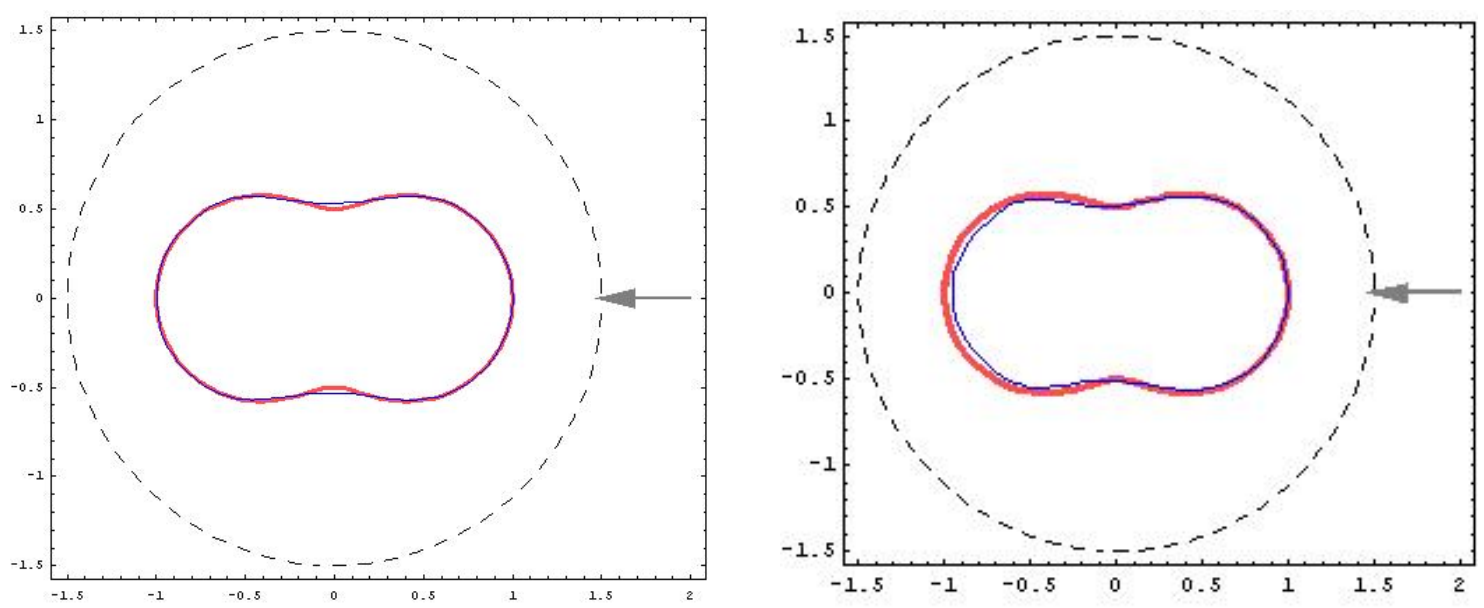

Figure 5.2: Reconstruction (in blue) of a peanut shaped sound-soft obstacle (in red) with exact data (left) and 5\% noise (right). The dashed line is the initial guess.
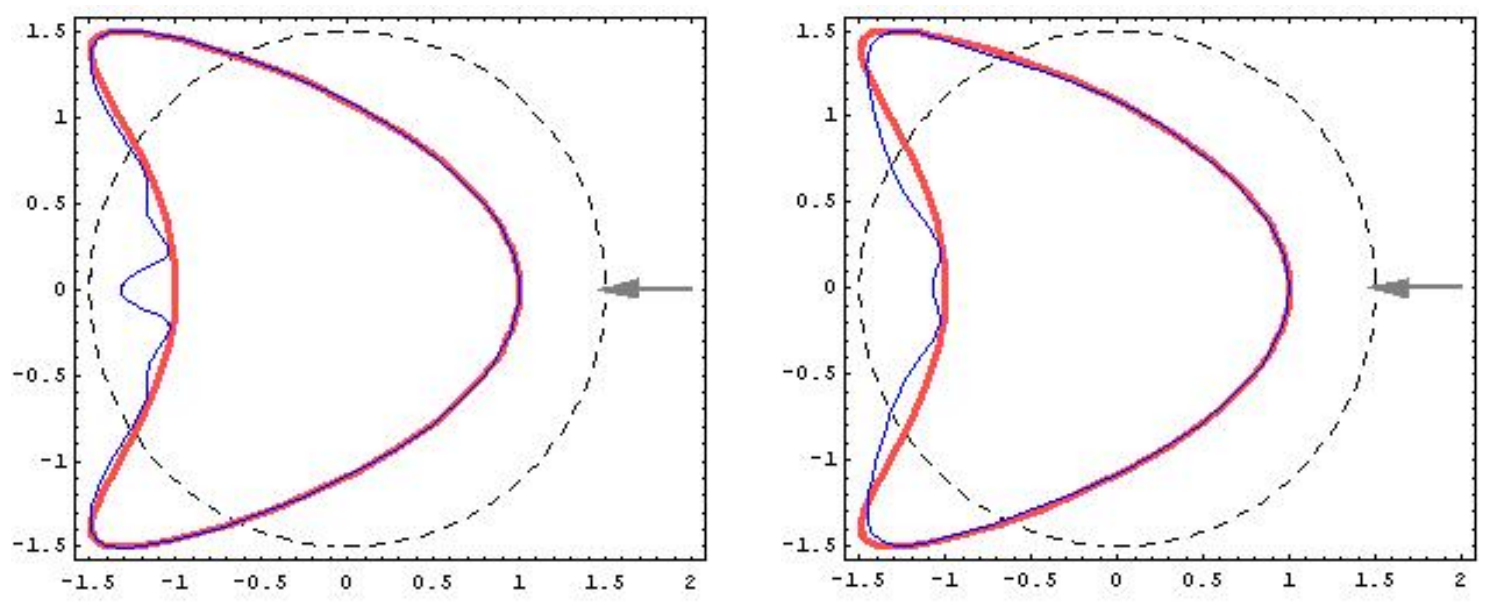

Figure 5.3: Reconstruction (in blue) of a kite shaped sound-soft obstacle (in red) with exact data (left) and 5\% noise (right). The dashed line is the initial guess.

As shown in figure 5.3, even for the kite shaped obstacle the performance is good. Both for noisy and non-noisy data, the reconstruction is worst in the convex part, that coincides with the shadow region.

Note that as mentioned in remark 3.1, the initial guess might be inside or outside the obstacle leading in both cases to successful reconstructions. However, as in general for a iterative method, convergence is only guaranteed for an initial guess sufficiently close to the obstacle. The numerical examples show the feasibility of the hybrid method as well as its robust behaviour with noisy data. 


\subsubsection{Inverse Problem for the Neumann Case}

For the Neumann case all the procedures follow in a very similar way. We again consider $\gamma_{n}$ to be the current approximation to the boundary and that the approximation is star-shaped and given by (5.20) and (5.21). We note that unlike the Dirichlet case and according to the analysis made in section 3.4, we represent the total field as a combined single-and double-layer potential

$$
u^{s}(x)=\int_{\gamma_{n}}\left(\frac{\partial \Phi(x, y)}{\partial \nu(y)}-i \eta \Phi(x, y)\right) \varphi(y) d s(y), \quad x \in \mathbb{R}^{2} \backslash \gamma_{n}
$$

for some coupling term $\eta>0$ and density $\varphi \in C^{1, \alpha}\left(\gamma_{n}\right)$. Though this representation is more complex to implement, one does not need any assumption on the wave number $k$. Having in mind the first step of the method, in order to reconstruct the total field $u$ one considers the Tikhonov regularized far-field equation given by

$$
\alpha_{n} \varphi^{(n)}+F_{\gamma, \infty}^{*} F_{\gamma, \infty} \varphi^{(n)}=F_{\gamma, \infty}^{*} u_{\infty} \quad \text { on } \Omega
$$

with

$$
\begin{aligned}
\left(F_{\gamma, \infty} \varphi\right)(\hat{x}) & :=\left(\left(K_{\gamma, \infty}-i \eta S_{\gamma, \infty}\right) \varphi\right)(\hat{x}) \\
& =\frac{e^{-i \pi / 4}}{\sqrt{8 \pi k}} \int_{\gamma}(k \hat{x} \cdot \nu(y)+\eta) e^{-i k \hat{x} \cdot y} d y
\end{aligned}
$$

In the parameterized form, one gets,

$$
\begin{array}{r}
\alpha_{n} \psi^{(n)}(s)+\frac{1}{8 \pi k} \int_{0}^{2 \pi} \Theta(t, s) e^{i k \hat{x}(t) \cdot z_{n}(s)}\left(\int_{0}^{2 \pi} \Theta(t, \tilde{t}) e^{-i k \hat{x}(t) \cdot z_{n}(\tilde{t})} \psi^{(n)}(\tilde{t})\left|z_{n}^{\prime}(\tilde{t})\right| d \tilde{t}\right) d t= \\
=\frac{e^{i \pi / 4}}{\sqrt{8 \pi k}} \int_{0}^{2 \pi} \Theta(t, s) e^{i k \hat{x}(t) \cdot z_{n}(s)} u_{\infty}(\hat{x}(t)) d t, \quad s \in[0,2 \pi]
\end{array}
$$

where $\psi^{(n)}(t)=\varphi^{(n)}\left(z_{n}(t)\right)$ for $t \in[0,2 \pi]$ and

$$
\Theta(t, s):=(k \hat{x}(t) \cdot \nu(s)+\eta), \quad t, s[0,2 \pi]
$$

Remark 5.1. Note that for the Neumann case one cannot get rid of the double integral arising, since there is no equivalent Bessel function integral representation as in the Dirichlet case. However, one can avoid this computationally more costly problem by discretizing the operator $F_{\gamma, \infty}$ into a matrix making use of the trapezoidal quadrature rule and then consider equation (5.26) in a matrix form. 
Using the trapezoidal rule (5.14) to approximate the integrals involved, again one ends up with a linear system

$$
\begin{array}{r}
\alpha_{n} \psi_{i}^{(n)}+\frac{\pi}{8 k N^{2}} \sum_{j=0}^{2 N-1} \Theta\left(t_{j}, t_{i}\right) e^{i k \hat{x}\left(t_{j}\right) \cdot z_{n}\left(t_{i}\right)}\left(\sum_{l=0}^{2 N-1} \Theta\left(t_{j}, t_{l}\right) e^{-i k \hat{x}\left(t_{j}\right) \cdot z_{n}\left(t_{l}\right)} \psi_{l}^{(n)}\left|z_{n}^{\prime}\left(t_{l}\right)\right|\right)= \\
=\frac{e^{i \pi / 4}}{\sqrt{8 k N}} \sum_{j=0}^{2 N-1} \Theta\left(t_{j}, t_{i}\right) e^{i k \hat{x}\left(t_{j}\right) \cdot z_{n}\left(t_{i}\right)} u_{\infty}\left(\hat{x}\left(t_{j}\right)\right),
\end{array}
$$

for $i=0, \ldots, 2 N-1$, which is solved in order to obtain the $\psi_{i}^{(n)}=\psi^{(n)}\left(z_{n}\left(t_{i}\right)\right)$. Making use of the quadrature rules mentioned to solve the direct problem, we then have the approximations to the scattered field $u^{s}$ and its normal derivative over $\gamma_{n}$ given by

$$
\begin{aligned}
u_{n}^{s}\left(z_{n}\left(t_{i}\right)\right) & :=\frac{\psi_{i}^{(n)}}{2}+\sum_{j=0}^{2 N-1}\left(R_{|i-j|}^{(N)} M_{1}\left(t_{i}, t_{j}\right)+\frac{\pi}{N} M_{2}\left(t_{i}, t_{j}\right)\right) \psi_{j}^{(n)}, \\
\left.\frac{\partial u_{n}^{s}}{\partial \nu}\left(z_{n}\left(t_{i}\right)\right)\right) & :=i \eta \frac{\psi_{i}^{(n)}}{2}+\sum_{j=0}^{2 N-1}\left(\frac{1}{\left|z^{\prime}\left(t_{i}\right)\right|} T_{|i-j|}^{(N)}+R_{|i-j|}^{(N)} \tilde{M}_{1}\left(t_{i}, t_{j}\right)+\frac{\pi}{N} \tilde{M}_{2}\left(t_{i}, t_{j}\right)\right) \psi_{j}^{(n)} .
\end{aligned}
$$

respectively, where the kernels are given by

$$
\begin{array}{cc}
M_{1}(s, t)=M_{1}^{K}(s, t)-i \eta M_{1}^{S}(s, t), & M_{2}(s, t)=M_{2}^{K}(s, t)-i \eta M_{2}^{S}(s, t), \\
\tilde{M}_{1}(s, t)=M_{1}^{T}(s, t)-i \eta M_{1}^{K^{*}}(s, t), & \tilde{M}_{2}(s, t)=M_{2}^{T}(s, t)-i \eta M_{2}^{K^{*}}(s, t) .
\end{array}
$$

This concludes the first step of the $n$-iteration of the hybrid method. For the second step one needs to solve the linearized equation (3.37). In this way one has

$$
\begin{aligned}
\left(h\left(t_{i}\right) \cdot \nu\left(t_{i}\right)\right)\left(H\left(t_{i}\right) \frac{\partial u_{n}}{\partial \nu}\left(z_{n}\left(t_{i}\right)\right)\right. & \left.-k^{2} u_{n}\left(z_{n}\left(t_{i}\right)\right)\right)-\frac{\partial}{\partial \tau}\left(h \cdot \nu\left(\frac{\partial u_{n}}{\partial \tau}\right) \circ z_{n}\right)\left(t_{i}\right)+ \\
& +\left(h\left(t_{i}\right) \cdot \tau\left(t_{i}\right)\right) \frac{\partial^{2} u_{n}}{\partial \tau \partial \nu}\left(z_{n}\left(t_{i}\right)\right)=-\frac{\partial u_{n}}{\partial \nu}\left(z_{n}\left(t_{i}\right)\right)
\end{aligned}
$$

for $i=0, \ldots, 2 N-1$. One uses the parametric representations of the normal and tangential vectors given by $\nu(s)=z^{\perp}(s) /\left|z^{\prime}(s)\right|$ and $\nu(s)=z^{\prime}(s) /\left|z^{\prime}(s)\right|$, respectively, and trigonometric differentiation on $u_{n}$ to compute the terms $\partial u_{n} / \partial \tau$ and $\partial^{2} u_{n} / \partial \tau^{2}$ and on $\partial u_{n} / \partial \nu$ to compute the term $\partial^{2} u_{n} / \partial \tau \partial \nu$. Like the Dirichlet case, one fits the coefficients $a_{j}^{(h)}, b_{j}^{(h)}, j=0, \ldots, N_{z}$ of the shift

$$
h(t)=\left(a_{0}^{(h)}+\sum_{j=1}^{N_{z}} a_{j}^{(h)} \cos j t+\sum_{j=1}^{N_{z}} b_{j}^{(h)} \sin j t\right)(\cos t, \sin t), \quad t \in[0,2 \pi]
$$


by a Levenberg-Marquardt step and gets a new approximation $\gamma_{n+1}$ with parameterization given by $z_{n+1}=z_{n}+h$. We then repeat the process while $\left\|\partial u_{n} / \partial \nu\right\|_{L^{2}\left(\gamma_{n}\right)}$ is decreasing.

For the numerical examples we considered wave number $k=3$ and again an incident plane field $u^{i}(x)=e^{i k x \cdot d}, d \in \Omega$. The increase of the wave number from $k=1$ to $k=3$ gives more accuracy in the reconstructions, especially for the peanut shaped obstacle, where the size of the obstacle is comparable to the wave length.

Again, the considered incident direction $d$ is represented in the figures by a grey arrow. We considered an approximation space for the radial parameterization defined by trigonometric polynomials of order less or equal to 10 and used the regularization parameter $\alpha_{n}=10^{-8} \times 0.5^{n}$ for Tikhonov regularization at the first step of each iteration $n$ of the method. The stopping criteria was achieved between 10 and 15 iterations for examples presented in this section. In this way, the sound-hard case seems to require more iterations than the sound-soft one.

We first considered the same obstacle with parameterization (5.23) that lies inside the approximation space. As one can see in figure 5.4 the reconstruction for the sound-hard case becomes slightly worst in the shadow region.
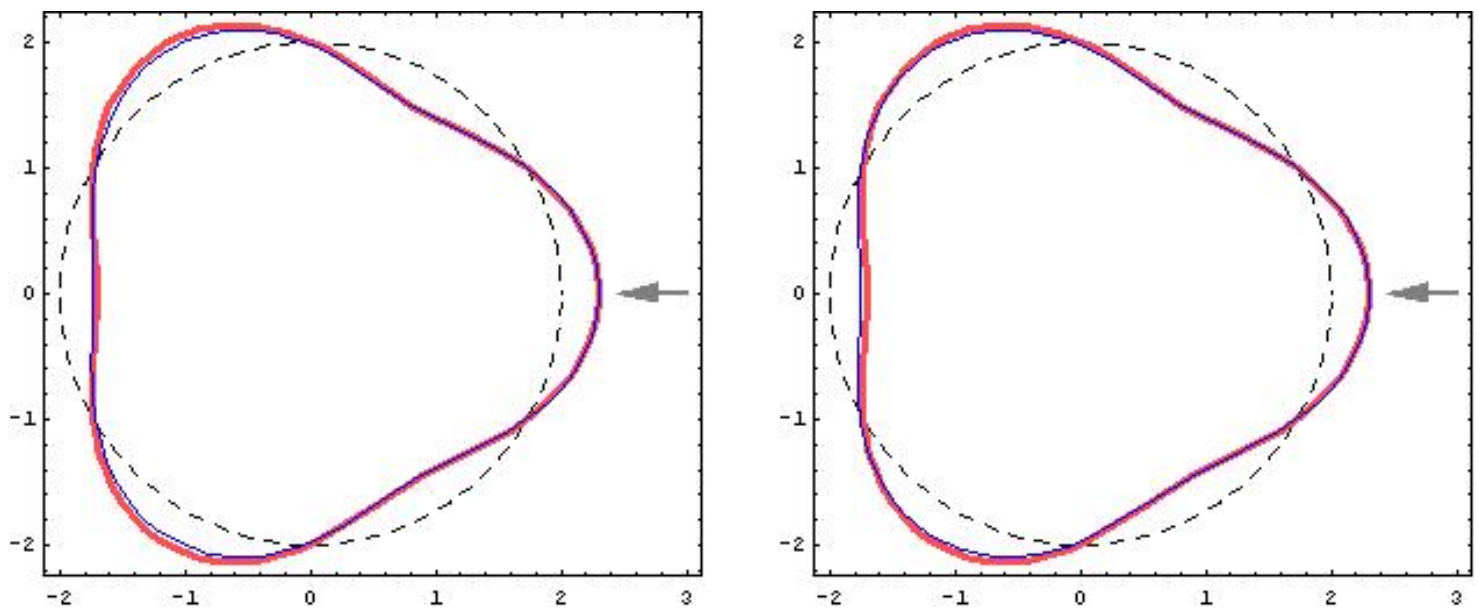

Figure 5.4: Reconstruction (in blue) of a sound-hard peanut shaped obstacle in the approximation space (in red) with exact data (left) and 5\% noise (right). The dashed line is the initial guess.

For the peanut shaped obstacle parameterized by (5.24) it is clear that even with exact data, the shadow region is not well reconstructed. Hence, the direction of the incident field becomes more important to get good reconstruction in the Neumann case. Moreover, the initial guess is also more important than in the Dirichlet case, since for the Neumann case there seems to be more local minimums to which the method might converge.

On figure 5.6 we present the reconstructions obtained for the kite-shaped obstacle. It is clear that the method does not work so well in this case, tending to a parallel sur- 

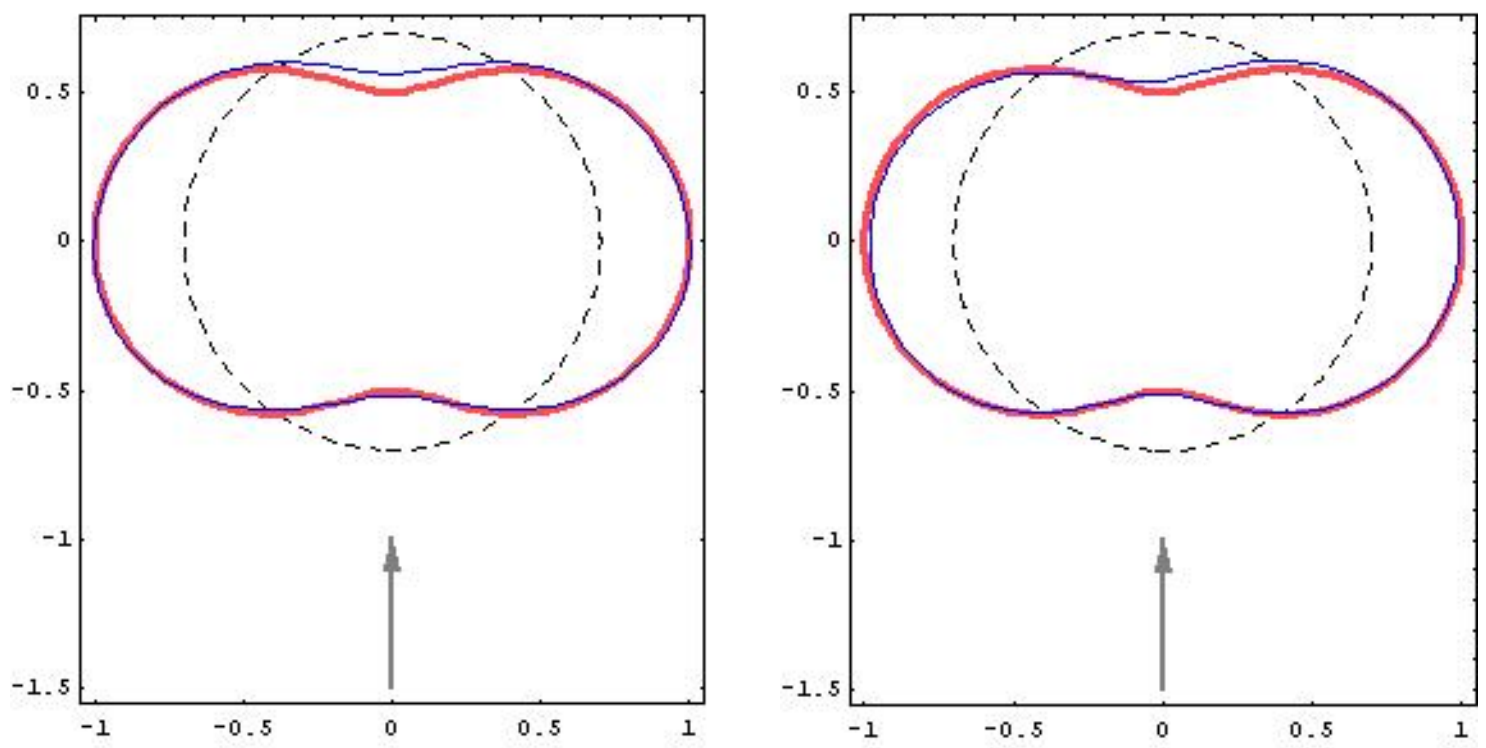

Figure 5.5: Reconstruction (in blue) of a sound-hard obstacle in the approximation space (in red) with exact data (left) and 5\% noise (right). The dashed line is the initial guess.
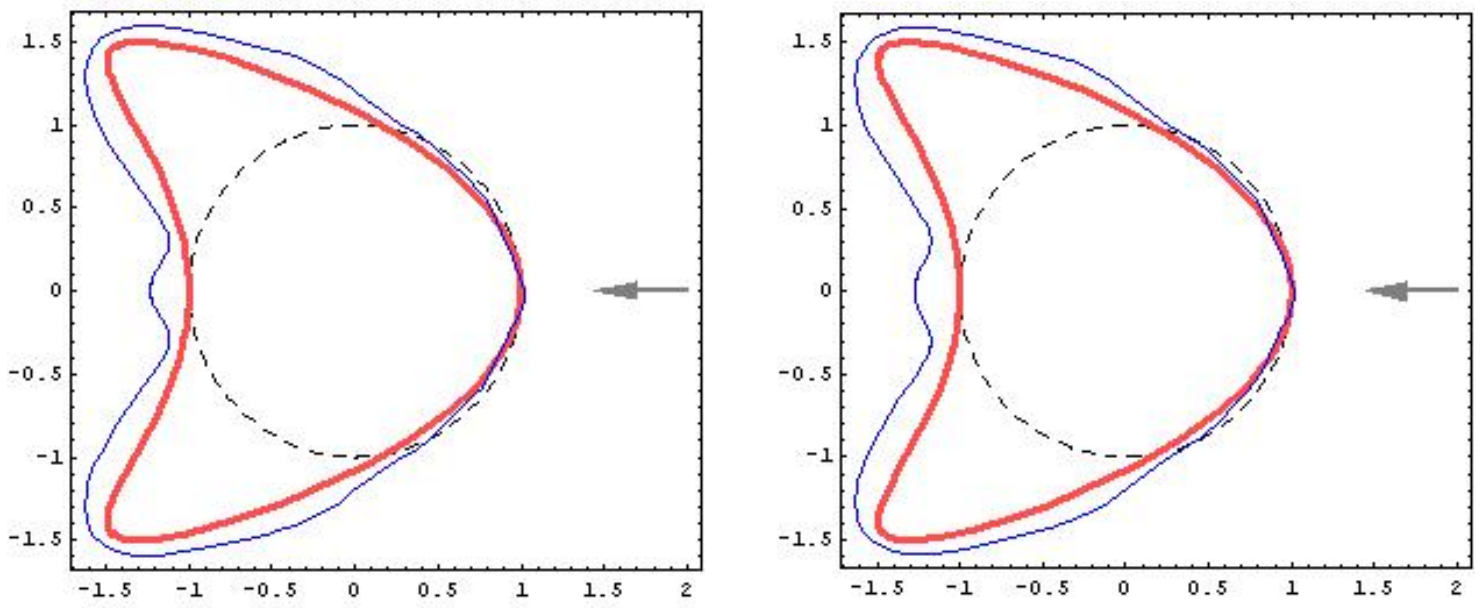

Figure 5.6: Reconstruction (in blue) of a sound-hard kite shaped obstacle in the approximation space (in red) with exact data (left) and 5\% noise (right). The dashed line is the initial guess.

face. We also note that for this last example the regularization parameter was increased to $\alpha_{n}=10^{-4} \times 0.5^{n}$ in order to get convergence. This might also explain the worst results, since the method becomes less accurate. Also due to this increasement, the differences between the exact and noisy case can hardly be seen. 


\subsubsection{Inverse Problem for the Robin Case}

In the Robin case one wants to recover not only the shape of the obstacle $\Gamma$ but also the impedance function $\lambda$. Therefore we consider that on the $n$-iteration we have a current approximation $\gamma_{n}$, parameterized by $z_{n}$, to the boundary $\Gamma$ and a current approximation $\zeta_{n}$ to the impedance $\lambda$. As we also use a combined single-and double-layer potential, the first step is exactly the same as the first step presented for the Neumann case, boiling down to solving (5.27) in order to obtain $\psi_{i}^{(n)}=\varphi^{(n)}\left(z_{n}\left(t_{i}\right)\right)$ for $i=0, \ldots, 2 N-1$. In the same way we have the approximations (5.28) and (5.29) respectively for the total field $u$ and its normal derivative over $\gamma_{n}$.

Before studying the second step, one issue still needs some discussion. Namely we need to discuss how to extend the impedance to a neighbourhood of $\gamma_{n}$, so that the operator $G_{R}$ defined in (3.53) and its Fréchet derivatives characterized in theorem 3.15 are well defined for all parameterizations $z$ in this neighbourhood. On the one hand, one way to do it would be to extend its values along the normal direction to $\gamma_{n}$, which is possible in a neighbourhood of the contour since the contour is assumed to be $C^{2}$-smooth (actually, by the approximation space defined by (5.20) and (5.21) it is even analytic). Though this idea does not impose any restrictions on the domain, as the characterization (3.56) of the Fréchet derivative of $G_{R}$ with respect to the parameterization depends on the gradient of $\zeta_{n}$, we would only be able to apply the method to continuously differentiable impedances, which is assuming more than we usually have in practice. On the other hand, assuming that the boundary of the domain is star-shaped, that is, it is parameterized by

$$
z(t)=\{r(t)(\cos t, \sin t): t \in[0,2 \pi]\}
$$

with some $2 \pi$-periodic positive $C^{2}$-function $r$, one could easily extend the impedance as a direction dependent function, that is, $\zeta=\zeta(\hat{x})$. In this way, the perturbations $h$ to the initial parameterization $z$ will also be star-shaped in the form of

$$
h(t)=\{q(t)(\cos t, \sin t): t \in[0,2 \pi]\}
$$

with some $2 \pi$-periodic $C^{2}$-function $q$ and therefore the term depending on $h \cdot \operatorname{grad} \zeta$ appearing on the characterization (3.56) of the Fréchet derivative of $G_{R}$ with respect to the parameterization $z$ will vanish since we have

$$
\operatorname{grad} \zeta \cdot h=\frac{\partial \zeta}{\partial \theta} \theta \cdot h=0
$$

where $\theta=\hat{x}^{\perp}$. Therefore we get

$$
\begin{aligned}
\frac{\partial}{\partial z} G(z, \zeta) h= & -k^{2}(h \cdot \nu) u \circ z-\frac{\partial}{\partial \tau}\left(h \cdot \nu\left(\frac{\partial u}{\partial \tau} \circ z\right)\right)+(i \zeta+H)(h \cdot \nu) \frac{\partial u}{\partial \nu} \circ z \\
& +(h \cdot \tau)\left[\left(\frac{\partial^{2} u}{\partial \tau \partial \nu}+i \zeta \frac{\partial u}{\partial \tau}\right) \circ z\right]
\end{aligned}
$$


which can be applied requiring the solution $\lambda$ just to be continuous, since there is no dependence on derivatives of $\zeta$. Note that this choice of extension of the impedance is also in accordance with the formal argument on "uniqueness" at the end of section 2.3 Therefore we will consider this last choice.

For star-shaped domains, from the linearized equation (3.55) we end up with the following $2 N$ equations

$$
\begin{array}{r}
-k^{2} h\left(t_{i}\right) \cdot \nu\left(t_{i}\right) u_{n}\left(z_{n}\left(t_{i}\right)\right)-\frac{\partial}{\partial \tau}\left(h\left(t_{i}\right) \cdot \nu\left(t_{i}\right)\left(\frac{\partial u_{n}}{\partial \tau}\left(z_{n}\left(t_{i}\right)\right)\right)\right) \\
+\left(i \zeta_{n}\left(t_{i}\right)+H\left(t_{i}\right)\right) h\left(t_{i}\right) \cdot \nu\left(t_{i}\right) \frac{\partial u_{n}}{\partial \nu}\left(z_{n}\left(t_{i}\right)\right) \\
+h\left(t_{i}\right) \cdot \tau\left(t_{i}\right)\left[\left(\frac{\partial^{2} u_{n}}{\partial \tau \partial \nu}\left(z_{n}\left(t_{i}\right)\right)+i \zeta_{n}\left(t_{i}\right) \frac{\partial u_{n}}{\partial \tau}\left(z_{n}\left(t_{i}\right)\right)\right)\right] \\
+i \mu\left(t_{i}\right) u_{n}\left(z_{n}\left(t_{i}\right)\right)=-\frac{\partial u_{n}}{\partial \nu}\left(z_{n}\left(t_{i}\right)\right)+i \zeta_{n}\left(t_{i}\right) u_{n}\left(z_{n}\left(t_{i}\right)\right),
\end{array}
$$

for $i=0, \ldots, 2 N-1$, where we use the jump relations and trigonometric differentiation to compute the terms involved. We now fit the coefficients $a_{j}^{(h)}, b_{j}^{(h)}, j=0, \ldots, N_{z}$ and $a_{j}^{(\mu)}, b_{j}^{(\mu)}, j=0, \ldots, N_{\mu}$ of the shifts

$$
\begin{aligned}
& h(t)=\left(a_{0}^{(h)}+\sum_{j=1}^{N_{z}} a_{j}^{(h)} \cos j t+\sum_{j=1}^{N_{z}} b_{j}^{(h)} \sin j t\right)(\cos t, \sin t), \quad t \in[0,2 \pi] \\
& \mu(t)=\left(a_{0}^{(\mu)}+\sum_{j=1}^{N_{\mu}} a_{j}^{(\mu)} \cos j t+\sum_{j=1}^{N_{\mu}} b_{j}^{(h)} \sin j t\right), \quad t \in[0,2 \pi]
\end{aligned}
$$

to the previous linear system by a Levenberg-Marquardt step and get an updated approximation $\gamma_{n+1}$ parameterized by $z_{n+1}=z_{n}+h$ to the boundary $\Gamma$ and an updated approximation $\zeta_{n+1}=\zeta_{n}+\mu$ to the impedance $\lambda$. Note that now we need $N_{z}+N_{\mu}<N$ so that the system is overdetermined. We then repeat the process while $\left\|\partial u_{n} / \partial \nu+i \zeta_{n} u_{n}\right\|_{L^{2}\left(\gamma_{n}\right)}$ is decreasing.

For the numerical examples we considered the wave number $k=1$ and again an incident plane field with incident direction $d$ represented in the figures by a grey arrow. We considered an approximation space for the radial parameterization defined by trigonometric polynomials of order less than or equal to $N_{z}=6$ and for impedance defined by trigonometric polynomials of order less than or equal to $N_{p}=5$. We used the regularization parameter $\alpha_{n}=10^{-8} \times 0.5^{n}$ for Tikhonov regularization at the first step of each iteration $n$ of the method. The stopping criteria was achieved between 10 and 15 iterations for the examples presented, as for the Neumann case. 
We first considered an obstacle with parameterization (5.23) and an impedance function

$$
\lambda(t)=1-\cos t+0.5 \sin 2 t, \quad t \in[0,2 \pi],
$$

both lying inside the respective approximation space. As one can see in figure 5.7, the reconstruction is no longer perfect even for exact data. However, this is drawback better noticed in the impedance function than in the obstacle, getting even clearer with noisy data. Moreover, the approximation around the maximums of the impedance is in general worst than around the minimums, since the behaviour for high impedance is similar to the Dirichlet case, being the method not able to recover the exact value around that area. This behaviour will also be shown in the following two examples. We also note that the initial guess represents a crucial choice for the Robin case, since the impedance allows even more flexibility to create local minimums in the problem than in the previous ones where the boundary condition was fixed.
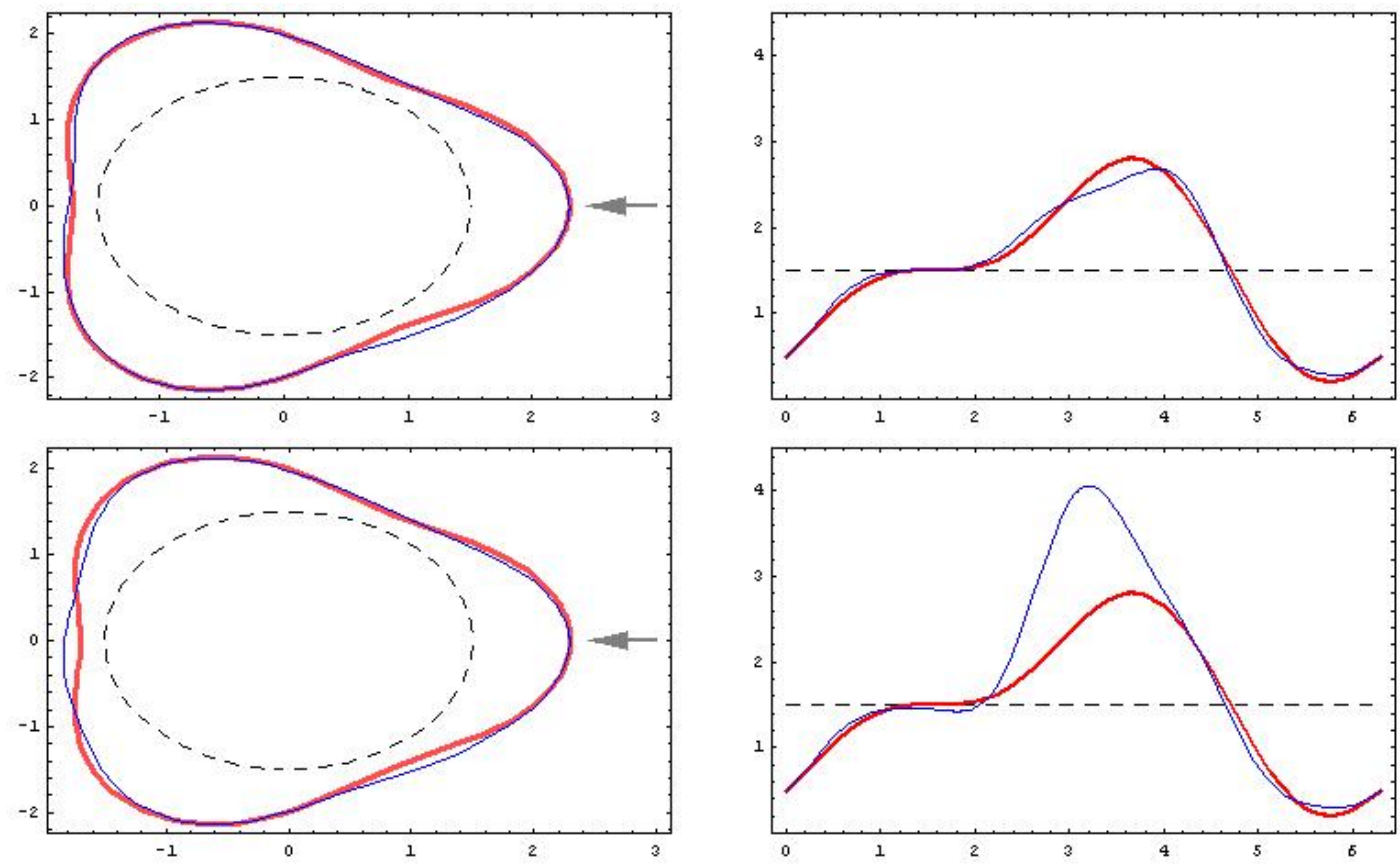

Figure 5.7: Reconstruction (in blue) of an obstacle (left in red) and the impedance function (right in red) with exact data (above) and 5\% noise (below). The dashed line is the initial guess.

For a second example we considered a peanut shaped obstacle parameterized by

$$
z(t)=3 \sqrt{\cos ^{2} t+0.25 \sin ^{2} t}(\cos t, \sin t), \quad t \in[2, \pi] .
$$

that is three times larger than the peanut shaped obstacle in the previous two sections. As mentioned in the previous section, better results are obtained when the size of the 
obstacle is larger than the wave number. We consider as impedance the function

$$
\lambda(t)=1+\sin ^{3} t, \quad t \in[0,2 \pi],
$$

that lies outside the approximation space. The numerical reconstructions are presented in figure 5.8. Again the reconstructions of the impedance and obstacle are worst in the shadow region.
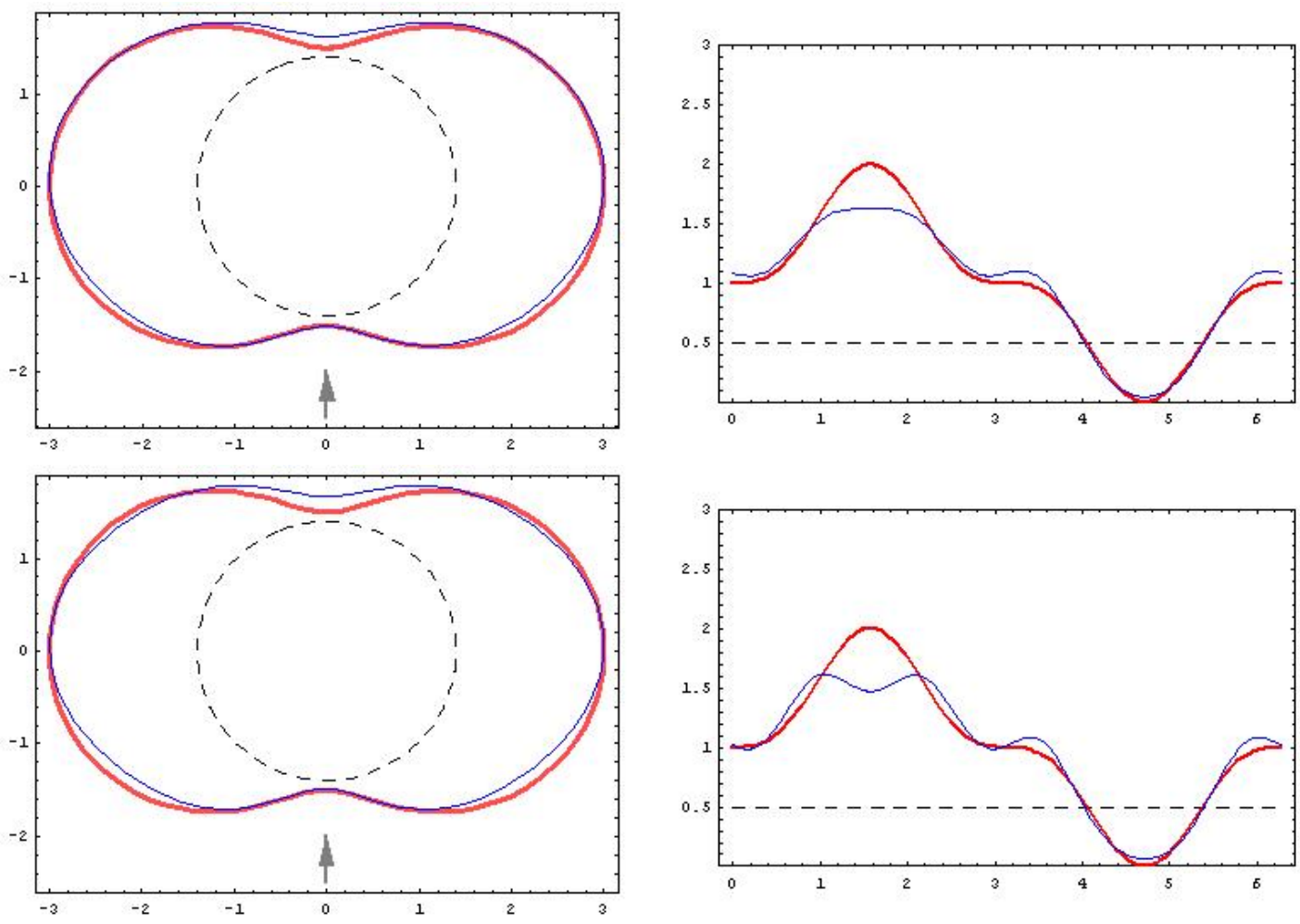

Figure 5.8: Reconstruction (in blue) of an obstacle (left in red) and the impedance function (right in red) with exact data (above) and 5\% noise (below). The dashed line is the initial guess.

As a last example in this section we present the reconstruction for the same peanutshaped obstacle parameterized by (5.32) and an impedance given by

$$
\lambda(t)=\left\{\begin{array}{cl}
0.6, & t \in[0, \pi], \\
0.6-0.6(t-\pi)(t-2 \pi), & t \in(\pi, 2 \pi]
\end{array}\right.
$$

with discontinuous derivative at $t=\pi$. To better reconstruct this impedance, since we are using a smooth approximation space we needed to increase the order of the approximation space for the impedance. In this way we consider as approximation space for 
the impedance the trigonometric polynomials of order less than or equal to $N_{l}=25$. The reconstruction is very good, presenting just some artifacts on the shadow region and around the maximums of the impedance that are amplified with noisy data.
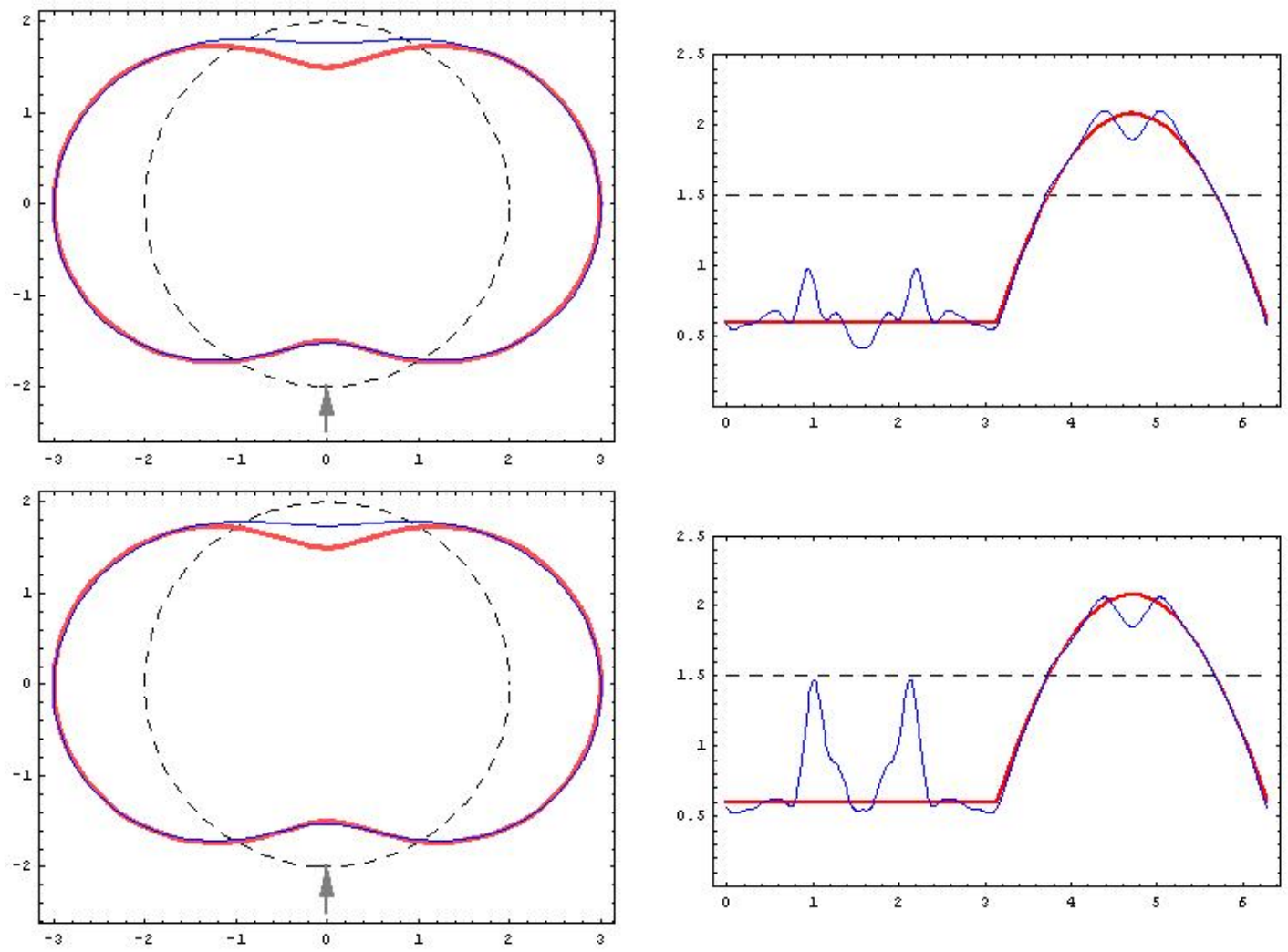

Figure 5.9: Reconstruction (in blue) of an peanut shaped obstacle (left in red) and an impedance $C^{0}$-function (right in red) with exact data (above) and 5\% noise (below). The dashed line is the initial guess.

\subsection{Three-dimensional case}

Due to simplicity, in three-dimensions we will only present reconstructions for the $\mathrm{Di}$ richlet boundary condition though to our opinion this method can be extended both to the Neumann and Robin boundary conditions following a similar procedure with proper changes. For the three-dimensional case the approach must be different since there is no straightforward simple quadrature rule available (similar to (5.13) for the twodimensional case) that deals with the singularity of the fundamental solution in $\mathbb{R}^{3}$. In this sense, the Nyström method loses some of its charm. A global approximation analogous 
to the trigonometric polynomials for the two dimensional case is also needed. Spherical harmonics are a proper choice for domains that can be mapped onto a sphere, since linear combinations of these functions defined over the unit sphere are dense in $L^{2}(\Omega)$ (e.g. [9]). For simplicity, in this section we denote the unit sphere by $\Omega:=\Omega_{3}$. Spherical harmonics are given by

$$
Y_{l}^{j}(\hat{x}(\theta, \phi))=(-1)^{(j+|j|) / 2} \sqrt{\frac{2 j+1}{4 \pi} \frac{(l-|j|) !}{(l+|j|) !}} P_{l}^{|j|}(\cos \theta) e^{i j \phi}
$$

for $l=1,2, \ldots$ and $j \leq|l|$, where $P_{l}^{|j|}$ are the associated Legendre polynomials and for $\hat{x} \in \Omega$ we consider the parametric form

$$
\hat{x}(\theta, \phi)=\{\sin \theta \cos \phi, \sin \theta \sin \phi, \cos \theta\}, \quad(\theta, \psi) \in[0, \pi] \times[0,2 \pi] .
$$

To generate the far-field pattern synthetic data, we will follow [15], where a fully discrete spectral method for the direct scattering problem is presented for smooth domains homeomorphic to a sphere. This method has superalgebraic convergence for analytic surfaces and right-hand sides. Its numerical implementation is presented in detail in [12]. Then we will use the quadrature rules arising from this method to numerically approximate the integral operators appearing while solving the inverse problem. Again we restrict our analysis to star-shaped domains of the form

$$
\gamma=\{z(\hat{x}):=r(\hat{x}) \hat{x}: \hat{x} \in \Omega\}
$$

for a radial function $r$ at least $C^{2}$-smooth over the unit sphere $\Omega$.

\subsubsection{Direct Problem}

We will now spend some lines on the basic ideas of the Galerkin method suggested in [15] to solve the direct problem. Representing the solution as a combined single-and doublelayer potential, due to the jump relations we arrive at the equation on the boundary

$$
\varphi+\mathcal{M} \varphi=f \quad \text { on } \Gamma
$$

with $f=-2 u^{i}$ and $\mathcal{M}=2(K-i \eta S)$. As in $\mathbb{R}^{2}$, the first goal is to split the singular part of the kernel $\mathcal{M}$ from the analytic one. Having this in mind we decompose $\mathcal{M}$ as

$$
\mathcal{M} \varphi(x)=\frac{1}{2 \pi} \int_{\Gamma}\left(\frac{1}{|x-y|} M_{1}(x, y)+M_{2}(x, y)\right) \varphi(y) d s(y)
$$


where the kernels $M_{1}$ and $M_{2}$ are smooth and, for the case of the combined single-and double-layer potential, are given by

$$
\begin{aligned}
& M_{1}(x, y)=\left(\frac{(x-y) \cdot \nu(y)}{|x-y|^{2}}-i \eta\right) \cos (k|x-y|)+k((x-y) \cdot \nu(y)) \mathcal{S}^{s}(x, y) \\
& M_{2}(x, y)=i \frac{(x-y) \cdot \nu(y)}{|x-y|^{2}}\left(\mathcal{S}^{s}(x, y)-k \cos (k|x-y|)\right)+\eta \mathcal{S}^{s}(x, y)
\end{aligned}
$$

where

$$
\mathcal{S}^{s}(x, y)= \begin{cases}\frac{\sin (k|x-y|)}{|x-y|}, & x \neq y \\ k, & x=y .\end{cases}
$$

For $\Gamma$ homeomorphic to the unit sphere $\Omega$, we can transform (5.34) in a equation over the unit sphere

$$
\psi+\mathcal{H} \psi=g \quad \text { on } \Omega
$$

where $\psi(\hat{x})=\varphi(z(\hat{x}))$ and $g(\hat{x})=f(z(\hat{x}))$. Note that we are in this case since we assume that $\Gamma$ is star-shaped, that is,

$$
\Gamma=\{z(\hat{x}):=r(\hat{x}) \hat{x}: \hat{x} \in \Omega\}
$$

with some $r \in C^{2}(\Omega)$. The parametric integral operator $\mathcal{H}$ can be decomposed as

$$
\begin{aligned}
\mathcal{H} \psi(\hat{y}) & =\frac{1}{2 \pi} \int_{\Omega}\left(\frac{1}{|\hat{x}-\hat{y}|} H_{1}(\hat{x}, \hat{y})+H_{2}(\hat{x}, \hat{y})\right) \psi(\hat{y}) d s(\hat{y}) \\
& =\frac{1}{2 \pi}\left(\mathcal{H}_{1} \psi(\hat{y})+\mathcal{H}_{2} \psi(\hat{y})\right),
\end{aligned}
$$

where we define the integral operator $\mathcal{H}_{1}$ with singular kernel and the integral operator $\mathcal{H}_{2}$ with smooth kernel as

$$
\begin{aligned}
\mathcal{H}_{1} \psi(\hat{y}) & =\int_{\Omega} \frac{1}{|\hat{x}-\hat{y}|} H_{1}(\hat{x}, \hat{y}) \psi(\hat{y}) d s(\hat{y}) \\
\mathcal{H}_{2} \psi(\hat{y}) & =\int_{\Omega} H_{2}(\hat{x}, \hat{y}) \psi(\hat{y}) d s(\hat{y})
\end{aligned}
$$

where the parameterized kernels are given by

$$
\begin{aligned}
& H_{1}(\hat{x}, \hat{y})=M_{1}(z(\hat{x}), z(\hat{y})) \mathcal{R}(\hat{x}, \hat{y}) J_{z}(\hat{y}) \\
& H_{2}(\hat{x}, \hat{y})=M_{2}(z(\hat{x}), z(\hat{y})) J_{z}(\hat{y})
\end{aligned}
$$


for

$$
\mathcal{R}(\hat{x}, \hat{y})=\frac{|\hat{x}-\hat{y}|}{|z(\hat{x})-z(\hat{y})|}
$$

and where the Jacobian of the transformation is be given by

$$
J_{z}=r \sqrt{r^{2}+|\nabla r|^{2}}
$$

with the gradient $\nabla r$ of $r$ over the unit sphere $\Omega$. The basic idea of [15] is to solve (5.36) by a Galerkin method seeking a solution $\psi_{N}$ to

$$
\left(\psi_{N}, \Psi\right)+\left(\mathcal{H} \psi_{N}, \Psi\right)=(g, \Psi) \quad \text { for all } \Psi \in \mathbb{P}_{N-1}
$$

where $\mathbb{P}_{N-1}$ is the space of linear combinations of spherical harmonics of order less than $N$. One now constructs a discrete version of the scalar product over $\Omega$

$$
\left(\Psi_{1}, \Psi_{2}\right)_{m}:=Q_{m}\left(\Psi_{1} \overline{\Psi_{2}}\right) \approx \int_{\Omega} \Psi_{1}(\hat{x}) \overline{\Psi_{2}(\hat{x})} d s(\hat{x})=\left(\Psi_{1}, \Psi_{2}\right)
$$

where $Q_{m}$ is a quadrature rule over the unit sphere $\Omega$. We consider the $m:=2 N^{2}$-point Gauss-trapezoidal rule (e.g. $[9,12])$ given by

$$
Q_{m}(\Psi)=\frac{\pi}{N} \sum_{j=1}^{N} \sum_{k=0}^{2 N-1} \alpha_{j} \Psi\left(\hat{x}_{j k}\right)
$$

for $\hat{x}_{j k}=\hat{x}\left(\theta_{j}, \phi_{k}\right)$. In the direction $\phi$ one uses the trapezoidal rule for periodic functions with equidistant points $\phi_{k}=k \pi / N, k=0, \ldots, 2 N-1$. For direction $\theta$ one chooses the Gauss rule with integration points $\theta_{j}=\arccos t_{j}, j=1, \ldots, N$ and weights

$$
\alpha_{j}=\frac{2\left(1-t_{j}^{2}\right)}{\left[N P_{N-1}\left(t_{j}\right)\right]^{2}}, \quad j=1, \ldots, N
$$

where $-1<t_{1}<t_{2}<\cdots<t_{N}<1$ denote the $N$ zeros of the Legendre polynomial $P_{N}$ of order $N$ in the interval $(-1,1)$. The quadrature rule $Q_{m}$ is exact for spherical polynomials of degree $2(N-1)$. In accordance with the discrete scalar product, we can also define the corresponding discrete orthogonal projection operator $\mathcal{P}_{N}: C(\Omega) \rightarrow \mathbb{P}_{N-1}$ given by

$$
\begin{aligned}
\mathcal{P}_{N} \Psi & =\sum_{l=0}^{N-1} \sum_{n=-l}^{l}\left(\Psi, Y_{l}^{n}\right)_{m} Y_{l}^{n} \\
& =\frac{\pi}{N} \sum_{j=0}^{N} \sum_{k=0}^{2 N-1} \alpha_{j} \Psi\left(\hat{x}_{j k}\right) \sum_{l=0}^{N-1} \sum_{n=-l}^{l} Y_{l}^{-n}\left(\hat{x}_{j k}\right) Y_{l}^{n}
\end{aligned}
$$


which satisfies $\mathcal{P}_{N} \Psi_{N-1}=\Psi_{N-1}$ for every $\Psi_{N-1} \in \mathbb{P}_{N-1}$ (for details see [61]), where again $\mathbb{P}_{N}$ holds for the spherical polynomials of order less than or equal to $N$ and is generated by the spherical harmonics of the same order.

As the integral kernel $\mathcal{H}$ has a singular part $\mathcal{H}_{1}$ and a smooth part $\mathcal{H}_{2}$ we treat each of them in a separate way.

For the singular part we introduce a change of coordinate system in $\Omega$, in order to take the singularity to the north pole. In this way we consider the orthogonal continuous transformation $T_{\hat{x}}$ that maps $\hat{x}$ to the north pole $\hat{n}:=(0,0,1)$ given by

$$
T_{\hat{x}}=P(\phi) Q(-\theta) P(-\phi)
$$

for $\hat{x}$ given by (5.33), where the $3 \times 3$ matrices $P(\psi)$ and $Q(\psi)$, that correspond to positive rotations by $\psi$ around the $z$-axis and $y$-axis, respectively, are given by

$$
P(\psi):=\left[\begin{array}{ccc}
\cos \psi & -\sin \psi & 0 \\
\sin \psi & \cos \psi & 0 \\
0 & 0 & 1
\end{array}\right], \quad Q(\psi):=\left[\begin{array}{ccc}
\cos \psi & 0 & \sin \psi \\
0 & 1 & 0 \\
-\sin \psi & 0 & \cos \psi
\end{array}\right] .
$$

We also define the transformation $\mathcal{T}_{\hat{x}}$ such that

$$
\mathcal{T}_{\hat{x}} \Psi(\hat{y})=\Psi\left(T_{\hat{x}}^{-1} \hat{y}\right), \quad \Psi \in C(\Omega), \hat{y} \in \Omega
$$

and its bivariate analog

$$
\mathcal{T}_{\hat{x}} \Psi\left(\hat{y}_{1}, \hat{y}_{2}\right)=\Psi\left(T_{\hat{x}}^{-1} \hat{y}_{1}, T_{\hat{x}}^{-1} \hat{y}_{2}\right), \quad \Psi \in C(\Omega \times \Omega), \hat{y}_{1}, \hat{y}_{2} \in \Omega
$$

From the orthogonality of $T_{\hat{x}}$ we have that

$$
\mathcal{H}_{1} \psi(\hat{x})=\int_{\Omega} \frac{1}{|\hat{n}-\hat{y}|} \mathcal{T}_{\hat{x}} H_{1}(\hat{n}, \hat{y}) \mathcal{T}_{\hat{x}} \psi(\hat{y}) d s(\hat{y})
$$

With the transformation $\mathcal{T}_{\hat{x}}$ and using polar coordinates for computing $\mathcal{H}_{1}$, the singularity of the denominator quantity $|\hat{n}-\hat{y}|=2 \sin (\theta / 2)$ is cancelled out by the surface element $d s(\hat{y})=\sin \theta d \theta d \phi$, that corresponds to the polar coordinate transformation. Moreover, it turns out that the mapping $(\theta, \phi) \mapsto \mathcal{T}_{\hat{x}} H_{1}(\hat{n}, \hat{y})$ is smooth (e.g. [15, lem. 4.6]). These two aspects indicate the crucial importance of the use of the rotated coordinate system in (5.42).

As this latter mapping $(\theta, \phi) \mapsto \mathcal{T}_{\hat{x}} H_{1}(\hat{n}, \hat{y})$ is continuous it makes sense to interpolate the integrand term $\mathcal{T}_{\hat{x}} H_{1}(\hat{n}, \hat{y}) \mathcal{T}_{\hat{x}} \psi(\hat{y})$ using the interpolation operator $\mathcal{P}_{N^{\prime}}$ defined in (5.41), for some $N^{\prime} \in \mathbb{N}$. Accordingly, using the $m^{\prime}=2 N^{\prime 2}$-point quadrature rule $Q_{m^{\prime}}$ defined in (5.40) with $N$ replaced by $N^{\prime}$, which is exact for spherical polynomials of de- 
gree $2\left(N^{\prime}-1\right)$, we get an approximation $\mathcal{H}_{1, N^{\prime}}$ to $\mathcal{H}_{1}$ given by

$$
\begin{aligned}
\mathcal{H}_{1}(\hat{x}) \approx \mathcal{H}_{1, N^{\prime}}(\hat{x}) & :=\int_{\Omega} \frac{1}{|\hat{n}-\hat{y}|} \mathcal{P}_{N^{\prime}}\left(\mathcal{T}_{\hat{x}} H_{1}(\hat{n}, .) \mathcal{T}_{\hat{x}} \psi(.)\right)(\hat{y}) d s(\hat{y}) \\
& =\sum_{l=0}^{N^{\prime}-1} \sum_{j=-l}^{l}\left(\mathcal{T}_{\hat{x}} H_{1}(\hat{n}, .) \mathcal{T}_{\hat{x}} \psi(.), Y_{l}^{j}(.)\right)_{m^{\prime}} \int_{\Omega} \frac{Y_{l}^{j}(\hat{y})}{|\hat{n}-\hat{y}|} d s(\hat{y}) \\
& =\sum_{l=0}^{N^{\prime}-1} \sum_{j=-l}^{l} \frac{4 \pi}{2 l+1}\left(\mathcal{T}_{\hat{x}} H_{1}(\hat{n}, .) \mathcal{T}_{\hat{x}} \psi(.), Y_{l}^{j}(.)\right)_{m^{\prime}} Y_{l}^{j}(\hat{n}),
\end{aligned}
$$

where we made use of the property (e.g. [9, pp.80])

$$
\int_{\Omega} \frac{\Psi_{n}(\hat{y})}{|\hat{x}-\hat{y}|} d s(\hat{y})=\frac{4 \pi}{2 n+1} \Psi_{n}(\hat{x}), \quad \Psi_{n} \in \mathbb{P}_{n}, \hat{x} \in \Omega .
$$

From the definition of $Q_{m^{\prime}}$ and the addition theorem for spherical harmonics of order $l$ (e.g. [9, thm.2.8])

$$
\sum_{j=-l}^{l} Y_{l}^{j}(\hat{x}) \overline{Y_{l}^{j}(\hat{y})}=\frac{2 l+1}{4 \pi} P_{n}(\hat{x} \cdot \hat{y})
$$

we can finally write our approximation $\mathcal{H}_{1, N^{\prime}}$ to $\mathcal{H}_{1}$ as

$$
\mathcal{H}_{1, N^{\prime}}(\hat{x})=\sum_{j^{\prime}=0}^{N^{\prime}} \sum_{k^{\prime}=0}^{2 N^{\prime}-1} \beta_{j^{\prime}} \mathcal{T}_{\hat{x}} H_{1}\left(\hat{n}, \hat{x}_{j^{\prime} k^{\prime}}^{\prime}\right) \mathcal{T}_{\hat{x}} \psi\left(\hat{x}_{j^{\prime} k^{\prime}}^{\prime}\right)
$$

where

$$
\beta_{j^{\prime}}=\frac{\pi \alpha_{j^{\prime}}^{\prime}}{N^{\prime}} \sum_{n=0}^{N^{\prime}-1} P_{n}\left(t_{j^{\prime}}^{\prime}\right)
$$

and the quadrature points $\hat{x}_{j^{\prime} k^{\prime}}^{\prime}$ and the weights $\alpha_{j^{\prime}}^{\prime}$ correspond to the quadrature rule $Q_{m^{\prime}}$, that is, the same procedure as for (5.40) with $N$ replaced by $N^{\prime}$. In other words (5.43) represents a quadrature rule to approximate the singular part of (5.37), which will also be important later for the inverse problem.

As for the smooth part $\mathcal{H}_{2}$ of (5.37), again we get an approximation by interpolating the integrand

$$
\mathcal{H}_{2}(\hat{x}) \approx \mathcal{H}_{2, N^{\prime}}(\hat{x}):=\int_{\Omega} \mathcal{P}_{\mathcal{N}^{\prime}}\left(\mathcal{T}_{\hat{x}} H_{2}(\hat{n}, .) \mathcal{T}_{\hat{x}} \psi(.)\right)(\hat{y}) d s(\hat{y})
$$


and as $Q_{m^{\prime}}$ is exact for spherical polynomials of order less than or equal to $2\left(N^{\prime}-1\right)$ one gets

$$
\begin{aligned}
\mathcal{H}_{2, N^{\prime}}(\hat{x}) & =Q_{m^{\prime}}\left(\mathcal{T}_{\hat{x}} H_{2}(\hat{n}, .) \mathcal{T}_{\hat{x}} \psi(.)\right) \\
& =\frac{\pi}{N^{\prime}} \sum_{j^{\prime}=1}^{N} \sum_{k^{\prime}=0}^{2 N-1} \alpha_{j^{\prime}}^{\prime} \mathcal{T}_{\hat{x}} H_{2}\left(\hat{n}, \hat{x}_{j^{\prime} k^{\prime}}^{\prime}\right) \mathcal{T}_{\hat{x}} \psi\left(\hat{x}_{j^{\prime} k^{\prime}}^{\prime}\right) .
\end{aligned}
$$

One can now define an approximation to $\mathcal{H}$ in (5.36) as

$$
\mathcal{H} \approx \mathcal{H}_{N^{\prime}}:=\frac{1}{2 \pi}\left(\mathcal{H}_{1, N^{\prime}}+\mathcal{H}_{2, N^{\prime}}\right) \text {. }
$$

Now the Galerkin method (5.39) can be reformulated as finding $\psi_{N} \in \mathbb{P}_{N-1}$ such that

$$
\left(\psi_{N}, Y_{l}^{j}\right)_{m}+\left(\mathcal{H}_{\mathcal{N}^{\prime}} \psi_{N}, Y_{l}^{j}\right)_{m}=\left(g, Y_{l}^{j}\right)_{m} \quad \text { for } l=0,1, \ldots, N-1,|j| \leq l .
$$

Defining

$$
\psi_{N}(\hat{x})=\sum_{l=0}^{N-1} \sum_{j=-l}^{l} a_{l}^{j} Y_{l}^{j}(\hat{x}),
$$

from (5.45) one obtains a well posed $N^{2} \times N^{2}$ linear system on the coefficients $a_{l}^{j}$ again for $l=0,1, \ldots, N-1,|j| \leq l$, which is solved to obtain the density $\psi_{N}$ (see [12]). In order to obtain the far-field synthetic data one applies again a Gauss-trapezoidal quadradrature rule to approximate the integral corresponding to the far-field of a combined singleand double-layer potential given by

$$
\begin{aligned}
u_{\infty}(\hat{x}) & =\left(\left(K_{\infty}-i \eta S_{\infty}\right) \varphi\right)(\hat{x}) \\
& \approx Q_{m}\left(-\frac{i}{4 \pi}(k(\hat{x} \cdot \nu(z(.)))+\eta) e^{-i k \hat{x} \cdot z(.)} \psi_{N}(.) J_{z}(.)\right) .
\end{aligned}
$$

This method is super-algebraic convergent for analytic boundaries and right-hand sides $f$ (see [15]), for

$$
(1+a) N<n^{\prime}<b N
$$

for some constants $a, b$ real and positive. These computations were made considering $N=20$ and $N^{\prime}=24$, generating the far-field pattern at 800 points over the unit sphere $\Omega$.

\subsubsection{Inverse Problem for the Dirichlet Case}

Let us consider that $\gamma_{n}$ is our current approximation to the correct boundary $\Gamma$. We start by considering a single-layer representation for the scattered field $u^{s}$

$$
u^{s}(x)=\int_{\gamma_{n}} \Phi(x, y) \varphi(y) d s(y)
$$


which can be done under certain assumptions as already mentioned in section 3.3.

For the inverse problem one needs to collect some of the information used for the direct problem, namely the use of the quadrature rules. From the representation of the scattered field one arrives at a first kind far-field integral equation given by

$$
S_{\gamma_{n}, \infty} \varphi=u_{\infty} \quad \text { on } \Omega
$$

where the single layer far-field operator

$$
\left(S_{\gamma, \infty} \varphi\right)(\hat{x})=\frac{1}{4 \pi} \int_{\gamma} e^{-i k \hat{x} \cdot y} \varphi(y) d s(y), \quad \hat{x} \in \Omega
$$

with some abuse of notation can also be given by

$$
\left(S_{\gamma, \infty} \psi\right)(\hat{x})=\frac{1}{4 \pi} \int_{\Omega} e^{-i k \hat{x} \cdot z(\hat{x})} \psi(\hat{y}) J_{z}(\hat{y}) d s(y), \quad \hat{x} \in \Omega
$$

for $\psi(\hat{y})=\varphi(z(\hat{y}))$. As the operator $S_{\gamma_{n}, \infty}$ is compact and the right-hand side $u_{\infty}$ might be noisy, just as in the two-dimensional case one uses Tikhonov regularization to solve the previous equation. In this way we solve

$$
\left(\alpha_{n} I+S_{\gamma_{n}, \infty}^{*} S_{\gamma_{n}, \infty}\right) \varphi^{(n)}=S_{\gamma_{n}, \infty}^{*} u_{\infty} \quad \text { on } \Omega
$$

that reduces itself to

$$
\begin{aligned}
\alpha_{n} \psi^{(n)}(\hat{x})+\frac{1}{16 \pi^{2}} \int_{\Omega}\left(\int_{\Omega} e^{i k \hat{\xi} \cdot(z(\hat{x})-z(\hat{y}))} d s(\hat{\xi})\right) & \psi^{(n)}(\hat{y}) J_{z}(\hat{y}) d s(\hat{y})= \\
& =\frac{1}{4 \pi} \int_{\Omega} e^{i k \hat{\xi} \cdot z(\hat{x})} u_{\infty}(\hat{\xi}) d s(\hat{\xi})
\end{aligned}
$$

where again $\psi^{(n)}(\hat{y})=\varphi^{(n)}(z(\hat{y}))$, since the adjoint operator $S_{\gamma_{n}, \infty}^{*}$ is given by

$$
\left(S_{\gamma, \infty}^{*} \varphi\right)(y)=\frac{1}{4 \pi} \int_{\Omega} e^{i k \hat{x} \cdot y} \varphi(\hat{x}) d s(\hat{x}), \quad y \in \gamma .
$$

Making use of the Funk-Hecke formula (see [9, p. 32]) for spherical harmonics of order zero we get

$$
\int_{\Omega} e^{i k \hat{\xi} \cdot(z(\hat{x})-z(\hat{y}))} d s(\hat{\xi})=4 \pi \frac{\sin (k|z(\hat{x})-z(\hat{y})|)}{k|z(\hat{x})-z(\hat{y})|}
$$

and therefore (5.47) simplifies to

$$
\begin{aligned}
\alpha_{n} \psi^{(n)}(\hat{x})+\frac{1}{4 \pi k} \int_{\Omega}\left(\frac{\sin (k|z(\hat{x})-z(\hat{y})|)}{|z(\hat{x})-z(\hat{y})|}\right) & \psi^{(n)}(\hat{y}) J_{z}(\hat{y}) d s(\hat{y})= \\
& =\frac{1}{4 \pi} \int_{\Omega} e^{i k \hat{\xi} \cdot z(\hat{x})} u_{\infty}(\hat{\xi}) d s(\hat{\xi}) .
\end{aligned}
$$


Again looking for an approximation $\psi_{N}^{(n)} \in \mathbb{P}_{N-1}$ as in (5.46) to the density $\psi^{(n)}$ and as the integral kernels involved in (5.48) are continuous, one uses the Gauss-trapezoidal quadrature rule (5.40) with $m=800$ (in order to use the given data set) to arrive at a linear system on the coefficients $a_{l}^{j}$ of $\psi_{N}^{(n)}$. Solving this linear system, one can now obtain explicitly

$$
\psi_{N}^{(n)}=\sum_{l=0}^{N-1} \sum_{j=-l}^{l} a_{l}^{j} Y_{l}^{j}(\hat{x}), \quad \hat{x} \in \Omega .
$$

Numerically this was done considering $N=8$.

Preparing the second step, by the jump relations one obtains approximations for the total field $u$ over the contour $\gamma_{n}$ given by

$$
u_{n}(x)=u^{i}(x)+\int_{\Omega} \Phi(x, z(\hat{y})) \psi_{N}^{(n)}(\hat{y}) J_{z}(\hat{y}) d s(y), \quad x \in \gamma_{n}
$$

and for its normal derivative over the contour $\gamma_{n}$ given by

$$
\begin{aligned}
\frac{\partial u_{n}}{\partial \nu}(z(\hat{x}))= & \frac{\partial u^{i}}{\partial \nu}(z(\hat{x}))-\frac{\psi_{N}^{(n)}(\hat{x})}{2} \\
& +\int_{\Omega} \frac{\partial \Phi(z(\hat{x}), z(\hat{y}))}{\partial \nu(z(\hat{x}))} \psi_{N}^{(n)}(\hat{y}) J_{z}(\hat{y}) d s(y), \quad \hat{x} \in \Omega .
\end{aligned}
$$

To numerically compute the integrals one again decomposes the integral kernels involved in a singular and smooth part, in a way similar to (5.35). For instance, for the single layer operator

$$
\begin{aligned}
S \varphi(x) & =\int_{\gamma} \Phi(x, y) \varphi(y) d s(y) \\
& =\frac{1}{4 \pi} \int_{\gamma}\left(\frac{1}{|x-y|} M_{1}^{S}(x, y)+M_{2}^{S}(x, y)\right) \varphi(y) d s(y)
\end{aligned}
$$

we would have

$$
\begin{aligned}
& M_{1}^{S}(x, y)=\cos (k|x-y|) \\
& M_{2}^{S}(x, y)=i \mathcal{S}^{s}(x, y)
\end{aligned}
$$

while for

$$
\begin{aligned}
K^{*} \varphi(x) & =\int_{\gamma} \frac{\partial \Phi(x, y)}{\partial \nu(x)} \varphi(y) d s(y) \\
& =\frac{1}{4 \pi} \int_{\gamma}\left(\frac{1}{|x-y|} M_{1}^{K^{*}}(x, y)+M_{2}^{K^{*}}(x, y)\right) \varphi(y) d s(y)
\end{aligned}
$$


we would have

$$
\begin{aligned}
& M_{1}^{K^{*}}(x, y)=\frac{(y-x) \cdot \nu(x)}{|x-y|^{2}} \cos (k|x-y|)+k((y-x) \cdot \nu(x)) \mathcal{S}^{s}(x, y) \\
& M_{2}^{K^{*}}(x, y)=i \frac{(y-x) \cdot \nu(x)}{|x-y|^{2}}\left(\mathcal{S}^{s}(x, y)-k \cos (k|x-y|)\right) .
\end{aligned}
$$

Considering the parameterized kernels as in (5.38) with $M$ replaced by $M^{S}$ and $M^{K^{*}}$ respectively, with a similar procedure one arrives at approximations to both singular and analytic parts given by (5.43) and (5.44), respectively. This yields approximations to the total field $u$ and its normal derivative over $\gamma_{n}$.

To compute the tangential component of the gradient over $\gamma_{n}$ one uses the analog to trigonometric differentiation in $\mathbb{R}^{2}$, that is, one interpolates $u(z()$.$) defined in the unit$ sphere by spherical harmonics and uses the tangential gradient of the interpolation as approximation to $\nabla_{\tau} u$. In this way, using the decomposition

$$
\left.\operatorname{grad} u\right|_{\gamma_{n}}=\left.\nu \frac{\partial u}{\partial \nu}\right|_{\gamma_{n}}+\nabla_{\tau} u
$$

accordingly to (3.14), one solves

$$
\operatorname{grad} u_{n}(z(\hat{x})) \cdot(h(\hat{x}) \hat{x})=-u_{n}(z(\hat{x})), \quad \hat{x} \in \Omega
$$

in a least squares sense, in order to find a shift $h \in V_{M}$ where $V_{M}$ is the space of linear combinations of real parts of spherical harmonics with order less than or equal to $M$ given by

$$
V_{M}=\left\{h \in \mathbb{P}_{M}: h(\hat{x})=\sum_{l=0}^{M} \sum_{j=0}^{l} a_{l}^{j} \operatorname{Re}\left(Y_{l}^{j}(\hat{x})\right), a_{l}^{j} \in \mathbb{R}\right\},
$$

which is an approximation space of dimension $(M+1) \times(M+2) / 2$. We used 128 points over $\Omega$ for the least squares minimization and considered $M=8$.

The two steps are then iterated while $\left\|u_{n}\right\|_{L^{2}\left(\gamma_{n}\right)}$ is decreasing.

We have applied the method to several examples and the numerical reconstructions were quite satisfying. We considered the wave number $k=1$ and a plane incident wave with direction $d=(0,1,0)$. The incident direction is indicated by an arrow in the pictures for the reconstructions. All the reconstructions were made with $2 \%$ noise in the maximum norm on the far-field data and using as initial guess a circle of radius $4 Y_{0}^{0}$. As regularization parameter for Tikhonov regularization we used $\alpha_{n}=0.5^{n} \times 10^{-8}$.

As a first example, in figures 5.10 and 5.11 we present the reconstructions with exact and noisy data, respectively, for an acorn shaped obstacle with parameterization

$$
r(\theta, \phi)=0.6+\sqrt{4.25+2 \cos 3 \theta},
$$


for $\theta \in[0, \pi], \phi \in[0,2 \pi]$.

The reconstruction is very good, even with noisy data.

Solution

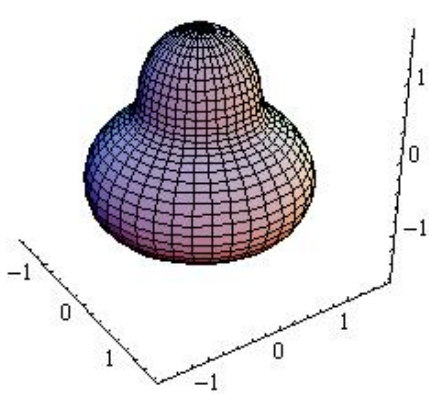

Approximation

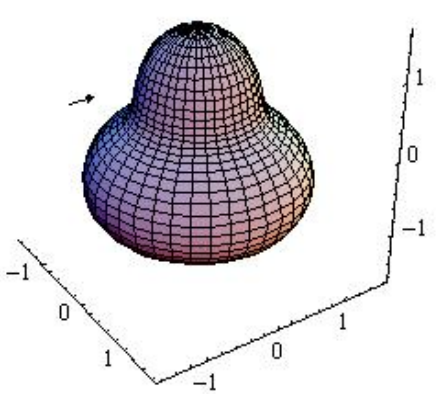

Error

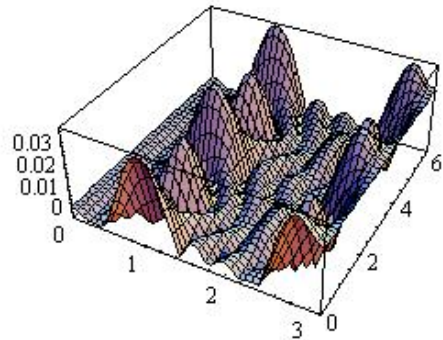

Figure 5.10: From left to right we present the acorn shaped obstacle, the reconstruction with exact data and the error in the parameterization space.

Solution

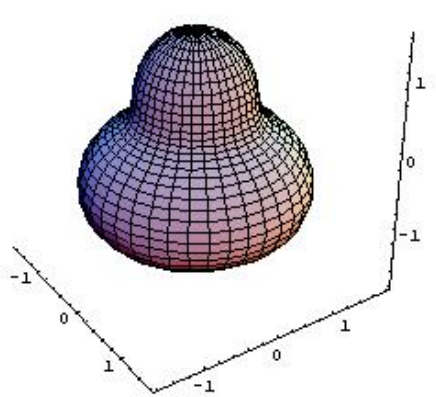

Approximation (2\% noise)

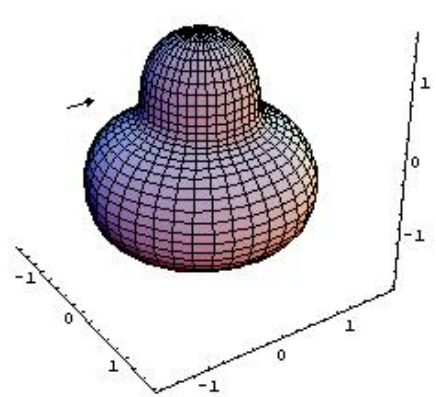

Error

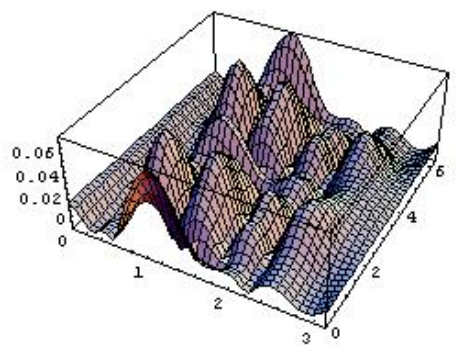

Figure 5.11: From left to right we present the acorn shaped obstacle, the reconstruction with $2 \%$ noise and the error in the parameterization space.

In figures 5.12 and 5.13 we present a pinched ball obstacle parameterized by

$$
r(\theta, \phi)=\sqrt{1.44+.5 \cos 2 \phi(\cos 2 \theta-1)}
$$

for $\theta \in[0, \pi], \phi \in[0,2 \pi]$. The reconstruction is almost perfect, as is the two-dimensional case. Again we note that it is worst in the non-convex part of the obstacle as usually occurs for numerical methods. 

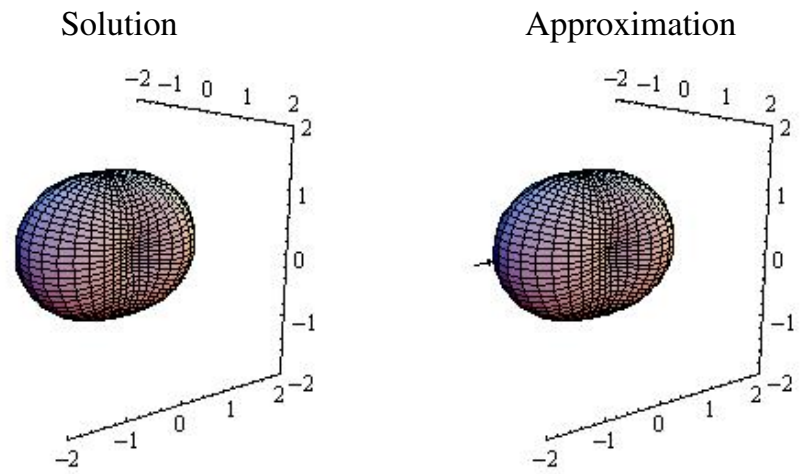

Error

Figure 5.12: From left to right we present the pinched-ball shaped obstacle, the reconstruction with exact data and the error in the parameterization space.
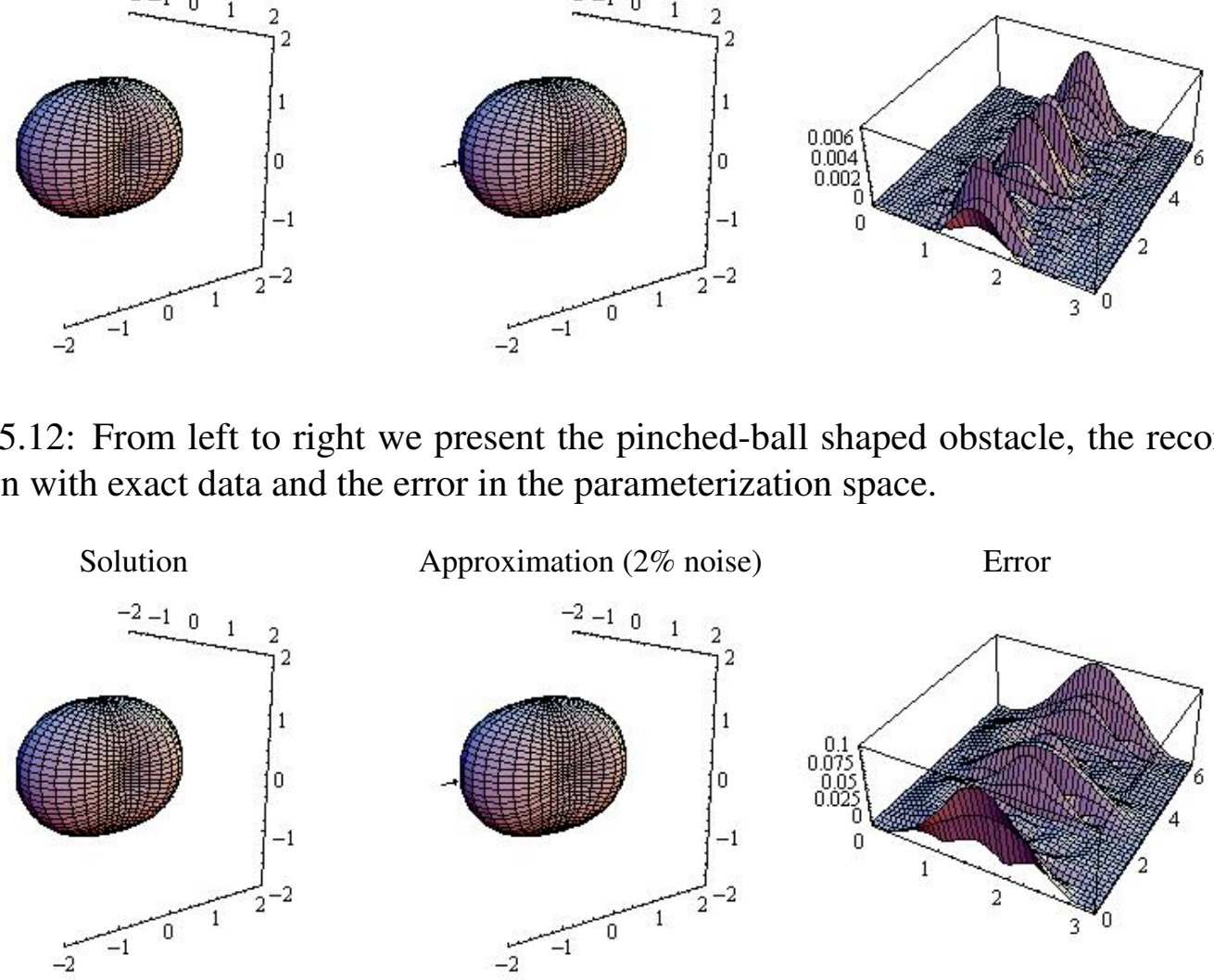

Figure 5.13: From left to right we present the pinched-ball shaped obstacle, the reconstruction with $2 \%$ noise and the error in the parameterization space.

To better illustrate this handicap, we present in figure 5.14 and 5.15 the reconstructions with exact and noisy data, respectively, for a pinched-acorn obstacle with parameterization

$$
r(\theta, \phi)=0.6 \sqrt{(1.44+0.5(\cos 2 \theta-1)(\cos 4 \phi))(4.25+2 \cos 3 \phi)},
$$

for $\theta \in[0, \pi], \phi \in[0,2 \pi]$. Again the non-convex part is not well-recovered, but it is clear that the method works quite fine even with an obstacle with high curvature as this one.

As a last example for the three-dimensional case, in figures 5.16 and 5.17 we present a cushion shaped obstacle parameterized by

$$
r(\theta, \phi)=\sqrt{0.8+0.5(\cos 2 \phi-1)(\cos 4 \theta-1)}
$$

for $\theta \in[0, \pi], \phi \in[0,2 \pi]$. Not that as in the two-dimensional case for the Dirichlet boundary condition, the reconstruction is the shadow region is still very good. 

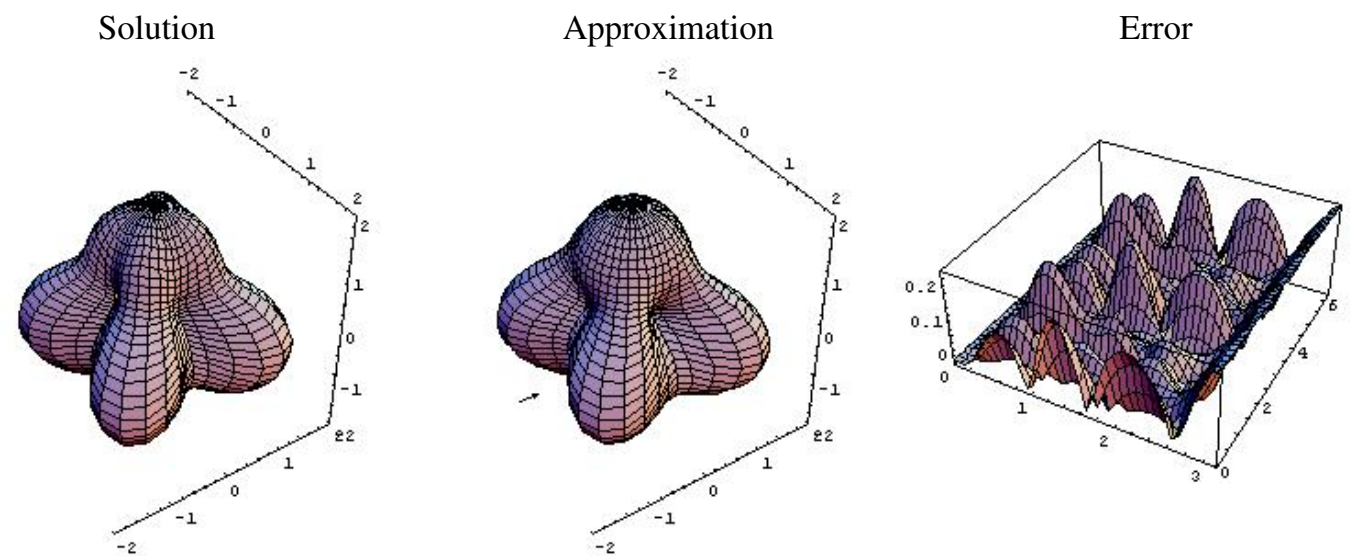

Figure 5.14: From left to right we present the pinched acorn shaped obstacle, the reconstruction with exact data and the error in the parameterization space.
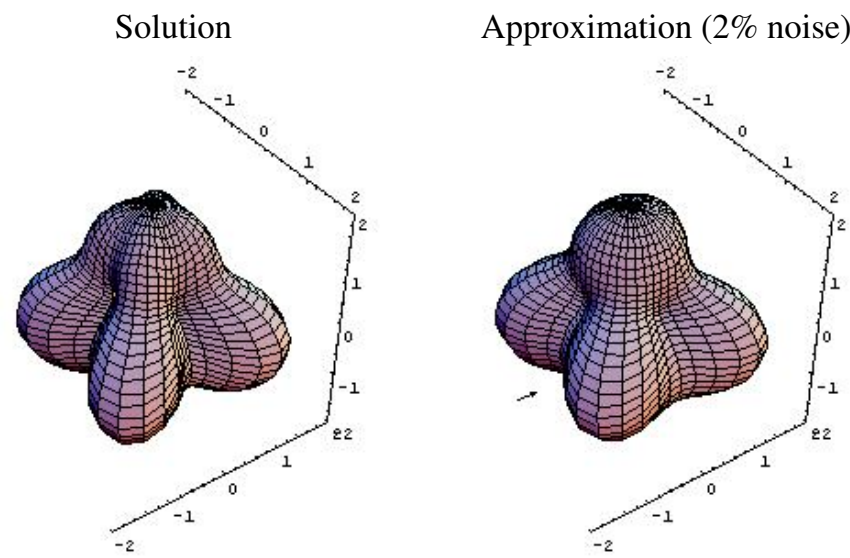

Error

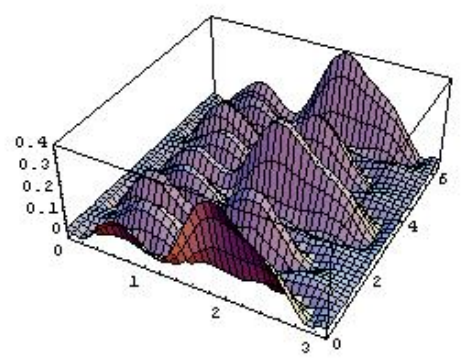

Figure 5.15: From left to right we present the pinched acorn shaped obstacle, the reconstruction with $2 \%$ noise and the error in the parameterization space.

The numerical reconstructions show the feasibility of the method and its robust behaviour with noisy data. 

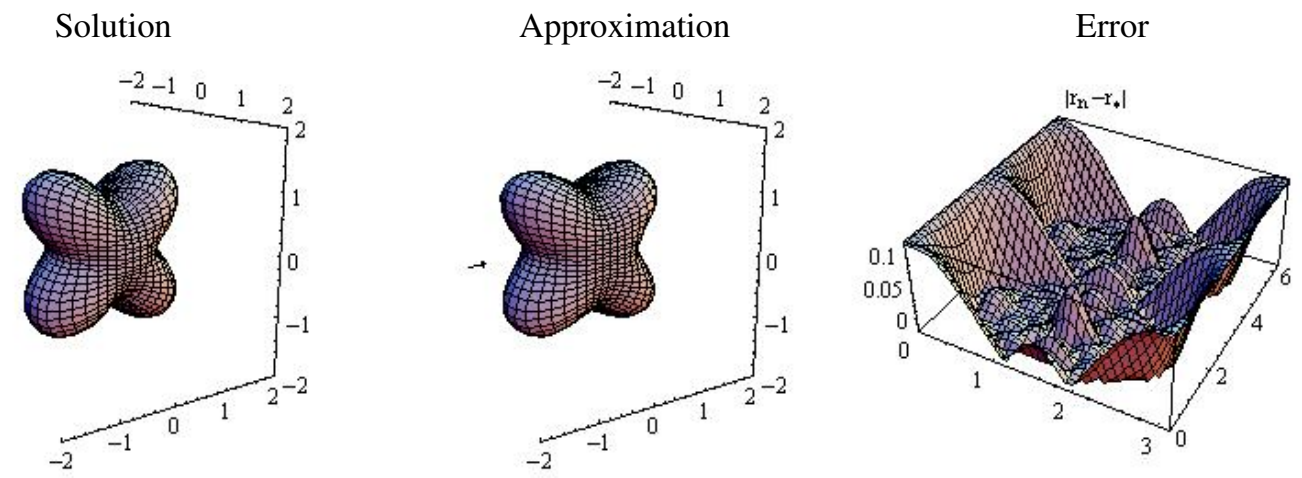

Figure 5.16: From left to right we present the cushion shaped obstacle, the reconstruction with exact data and the error in the parameterization space.
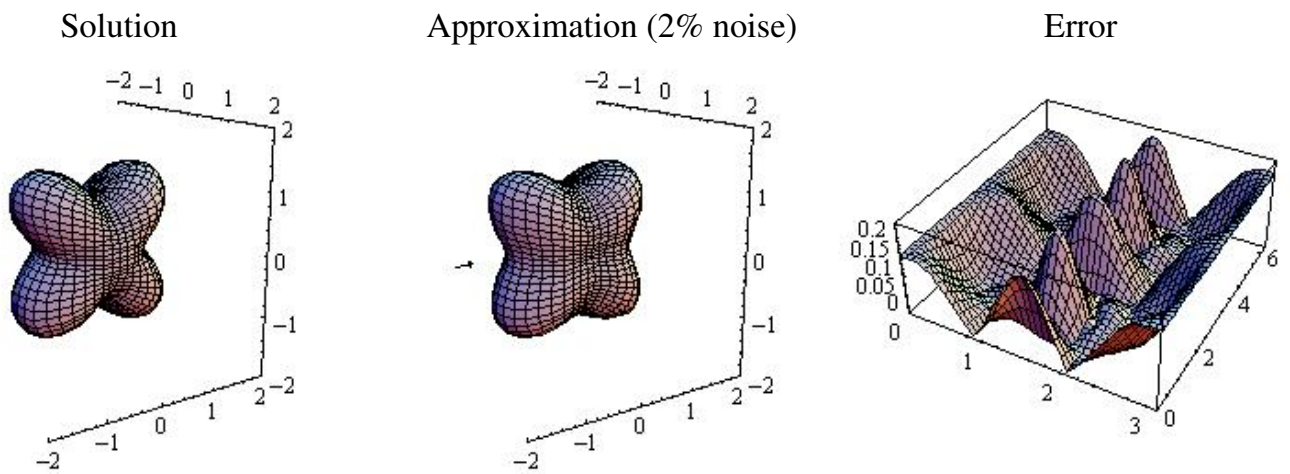

Figure 5.17: From left to right we present the cushion shaped obstacle, the reconstruction with $2 \%$ noise and the error in the parameterization space. 



\section{Chapter 6}

\section{Conclusions and Outlook}

In this last chapter we will present some conclusions on the work done and some perspectives for future work on the hybrid method.

The hybrid method proved itself to achieve good numerical reconstructions requiring only the far-field data for one single incident wave. The combination of ideas from decomposition and iterative methods generated a method with good numerical accuracy though requiring no forward solver. The hybrid method is closer to a decomposition method, since it takes care of the ill-posedness and non-linearity of the inverse problem in two different steps. In fact, the hybrid method presents some close connections to the Kirsch and Kress decomposition method [30, 31, 32], with the main difference in the role played by the surface $\gamma$, as referred to in remark 3.1. This way, the hybrid method is an iterative method that uses the idea of analytic continuation of the total field. This procedure does not need a forward solver in each iteration step, which reduces the computational costs. We also stress that the changes made on the Kirsch and Kress method to create the hybrid method allow an iterative procedure that gives more flexibility to the method. Namely, one no longer needs the initial guess to lie inside the obstacle, which implies less a priori knowledge. Moreover, the method can be used for the case of an unknown impedance. In this way it can be seen as an alternative to the iterative method [40], since it also reconstructs both the obstacle and the impedance simultaneously. Thus, the hybrid method achieved the goal of a good compromise between data required, computational costs and numerical accuracy. However, it also carries some disadvantages, such as needing a good initial guess to start the iterations from.

The characterization of the method was presented both from a theoretical and numerical point of view. As for the theoretical background, the application of the method was compared with two other problems - a related minimization problem and a related Newton method - for which convergence was proven. We achieved to prove convergence for the minimization problem for all considered boundary conditions, though there is a gap between theory and the implementation of the method. This approach also did not consider the linearization on the second step of the method nor the iterative procedure. 
In order to overcome this, we introduced a new approach to prove convergence, namely through a related Newton method. Though the latter approach faithfully represents the numerical implementation of the hybrid method, convergence results could only be obtained for sound-soft obstacles and assuming that the radial derivative of the total field does not have zeros over the boundary of the obstacle.

In what concerns numerical applications, we stress the importance of the characterization of the Fréchet derivative of the operator $G$ defined in (3.6). This characterization was given for the Dirichlet, Neumann and Robin boundary condition both in $\mathbb{R}^{2}$ and $\mathbb{R}^{3}$, using as primary tool the Taylor formula. Therefore the method can be implemented numerically in all these cases. These characterizations depend on the total field $u$ and its normal and tangential derivatives of, at most, second order over the considered contour. Hence, the numerical implementation needs to consider numerical procedures to compute these terms. Since the total field is represented as a layer potential, through the jump relations one gets explicit formulas to compute both the total field and its normal derivative over the contour. For smooth surfaces, the integrals are computed using exponential convergent quadrature rules both in $\mathbb{R}^{2}$ and $\mathbb{R}^{3}$. The tangential derivatives are then computed using trigonometric differentiation.

In $\mathbb{R}^{2}$ we first considered sound-soft and sound-hard obstacle numerical reconstructions. The numerical results were very good in both cases, though slightly better in the first case than in the second. Moreover, we also applied the method to inverse scattering for shape and impedance in $\mathbb{R}^{2}$. Concerning the obstacle reconstruction, the approximations were on the same level of accuracy as for the Neumann case. For the impedance the reconstructions were also good, though more sensitive to noisy data. In all cases the approximation in the shadow region was worse than in the illuminated area, especially for noisy data. In $\mathbb{R}^{3}$ we presented reconstructions for sound-soft obstacles. The reconstructions were again very good, which shows the feasibility of the method both in the two-and three-dimensional case. The robust behaviour of the method to noisy data is also exposed.

The idea of combining ideas from decomposition and iterative methods in order to create the hybrid method under consideration was suggested in [38]. In this work we presented its development through the past years and its current state of the art. The hybrid method was considered for potential theory [5] and inverse acoustic scattering as shown in the work at hand. It seems reasonable that this method can be extended and applied for other types of scattering, namely elastic or electromagnetic scattering. The ideas follow straightforwardly, though proper changes are required to obtain the new characterizations for the Fréchet derivative of the corresponding operator $G$. Moreover, these problems would impose more complexity in the numerical implementation, since one would have vector fields instead of scalar fields as in the acoustic case.

Also in a theoretical level some developments might still be done. Concerning the related minimization problem, it might be interesting to use or develop a theory for min- 
imization in two steps as a more realistic theoretical representation of the method. This would also imply a completely satisfying convergence proof for the Kirsch and Kress decomposition method. In this way we would have a closer representation to the numerical implementation of the method, as happens with the related Newton method. However, in the latter case, it would also be nice to have a criterion to know whether the radial derivative of the total field vanishes somewhere over the boundary. Note that this condition is important for the presented proof, though it is not clear under which conditions this can be assumed. We presented some quite restrictive condition in remark 4.9. Moreover, a similar convergence proof for the Neumann and Robin boundary conditions is still missing, since the given proof only works for the sound-soft case. This is due to the fact that we only have an explicit representation for the shift $h$ in this last case, since for the Neumann and Robin boundary condition the characterization of the Fréchet derivative of the operator $G$ depends on the derivatives of $h$. One way to try to overcome this problem would be to try to represent $h$ as a linear combination, say of trigonometric polynomials in $\mathbb{R}^{2}$. Although one might get a Newton iterative scheme on the coefficients of the numerical representation by this procedure, this implies to study the solution as the dimension of the trigonometric approximation space goes to infinity. However, one could start by using a finite dimensional space and assume that the solution is within the approximation space.

As shown in the previous lines, the hybrid method is still a challenging problem, both from a theoretical and from a numerical point of view. 



\section{Bibliography}

[1] G. Alessandrini and L. Rondi. Determining a sound-soft polyhedral scatterer by a single far field measurement. Proc. Amer. Math. Soc., 133:1685-1691, 2005.

[2] J. Bowman, T. Senior, and P. Uslenghi, editors. Electromagnetic and acoustic scattering by simple shapes. North-Holland Publishing Company, Amsterdam, 1969.

[3] F. Cakoni and D. Colton. The determination of the surface impedance of a partially coated obstacle from far-field data. SIAM J. Appl. Math., 64:709-723, 2004.

[4] F. Cakoni and D. Colton. Qualitative methods in inverse scattering theory. Springer, Berlin, 2006.

[5] R. Chapko and R. Kress. A hybrid method for inverse boundary values problems in potential theory. J. Inverse Ill-Posed Problems, 13:1-14, 2005.

[6] J. Cheng and M. Yamamoto. Uniqueness in inverse scattering problem within nontrapping polygonal obstacles with at most two incoming waves. Inverse Problems, 19:1361-1384, 2003.

[7] D. Colton and A. Kirsch. A simple method for solving inverse scattering problems in the resonance region. Inverse problems, 12:383-393, 1996.

[8] D. Colton and R. Kress. Integral Equation Methods in Scattering Theory. Wiley, Chichester, 1983.

[9] D. Colton and R. Kress. Inverse Acoustic and Electromagnetic Scattering Theory. Springer, Berlin, $2^{\text {nd }}$ edition, 1998.

[10] D. Colton and R. Kress. Using fundamental solutions in inverse scattering theory. Inverse Problems, 22:285-299, 2006.

[11] D. Colton and B. Sleeman. Uniqueness theorems for the inverse problem of acoustic scattering. IMA J. Appl. Math., 31:253-259, 1983. 
[12] M. Ganesh and I. Graham. A high order algorithm for obstacle scattering in three dimensions. J. Comp. Phys., 198:211-242, 2004.

[13] D. Gilbarg and N. Trudinger. Elliptic partial differential equations of second order. Springer, Berlin, $2^{\text {nd }}$ edition, 1998.

[14] D. Gintides. Local uniqueness for the inverse scattering problem in acoustics via the faber-krahn inequality. Inverse Problems, 21:1195-1205, 2005.

[15] I. Graham and I. Sloan. Fully discrete spectral boundary integral methods for helmholtz problems on smooth closed surfaces in $\mathbb{R}^{3}$. Numer. Math., 92:289-323, 2002.

[16] N. Grinberg. Factorization Method in Inverse Obstacle Scattering. Habilitation Thesis, Karlsruhe, 2004.

[17] J. Hadamard. Lectures on Cauchy's Problem in Linear Partial Differential Equations. New York, Dover, 1952.

[18] F. Hettlich. Fréchet derivatives in inverse obstacle scattering. Inverse problems, 11:371-382, 1995, (erratum, Inverse Problems 14, 204-210, 1998).

[19] F. Hettlich. The Domain derivative in Inverse Obstacle Problems. Habilitation Thesis, 1999.

[20] T. Hohage. Logarithmic convergence rates of the iteratively regularized GaussNewton method for an inverse potential and an inverse scattering problem. Inverse Problems, 13:1279-1299, 1997.

[21] T. Hohage. Convergence rates of a regularized newton method in sound-hard inverse scattering. SIAM J. Numer. Anal., 36:125-142, 1998.

[22] T. Hohage. Iterative Methods in Inverse Obstacle Scattering: Regularization Theory of Linear and Non-Linear Exponentially Ill-posed Problems. Dissertation, Linz, 1999.

[23] M. Ikehata. Reconstruction of an obstacle from the scattering amplitude at a fixed frequency. Inverse Problems, 14:949-954, 1998.

[24] V. Isakov. Inverse Problems for Partial Differential Equations. Springer-Verlag, New York, 1998.

[25] O. Ivanyshyn and R. Kress. Nonlinear integral equations for solving inverse boundary value problems for inclusions and cracks. Jour. Integral Equations and Appl., 18:13-38, 2006. 
[26] A. Kirsch. Properties of far-field operators in acoustic scattering. Math. Meth. Appl. Scie., 11 (no. 6):773-787, 1989.

[27] A. Kirsch. Surface gradients and continuity properties for some integral operators in classical scattering theory. Math. Methods Appl. Sci., 11 (no. 6):789-804, 1989.

[28] A. Kirsch. An Introduction to the Mathematical Theory of Inverse Problems. Springer-Verlag, New York, 1996.

[29] A. Kirsch. Characterization of the scattering obstacle by the spectral data of the far field operator. Inverse Problems, 14:1489-1512, 1998.

[30] A. Kirsch and R. Kress. On an integral equation of the first kind in inverse acoustic scattering. Inverse Problems (Cannon and Hornung, eds.), ISNM, 77:93-102, 1986.

[31] A. Kirsch and R. Kress. A numerical method for an inverse scattering problem. (Engl and Groetsch, eds.), Academic Press, Orlando,, pages 279-290, 1987.

[32] A. Kirsch and R. Kress. An optimization method in inverse acoustic scattering. Boundary Elements IX, Vol.3 Fluid Flow and Potential Applications (Brebbia et al., eds), Springer Verlag, Berlin Heidelberg New York, pages 3-18, 1987.

[33] A. Kirsch and R. Kress. Uniqueness in inverse obstacle scattering. Inverse Problems, 9:285-299, 1993.

[34] A. Kirsch, R. Kress, P. Monk, and A. Zinn. Two methods for solving the inverse acoustic scattering problem. Inverse Problems, 3:749-770, 1988.

[35] R. Kress. Minimizing the condition number of boundary integral operators in acoustic and electromagnetic scattering. Quart. J. Mech. Appl. Math., 38:323-341, 1985.

[36] R. Kress. On the numerical solution of a hypersingular integral equation in scattering theory. J. Comp. Appl. Math., 61:345-360, 1995.

[37] R. Kress. Numerical analysis. Springer, New York, 1998.

[38] R. Kress. Newton's method for inverse obstacle scattering meets the method of least squares. Inverse Problems, 19:91-104, 2003.

[39] R. Kress. Uniqueness and numerical methods in inverse obstacle scattering. (to appear).

[40] R. Kress and W. Rundell. Inverse scattering for shape and impedance. Inverse problems, 17:1075-1085, 2001. 
[41] R. Kress and W. Rundell. Nonlinear integral equations and the iterative solution for an inverse boundary value problem. Inverse problems, 21:1207-1223, 2005.

[42] R. Kress and P. Serranho. A hybrid method for two-dimensional crack reconstruction. Inverse Problems, 21:773-784, 2005.

[43] R. Kress and P. Serranho. A hybrid method for sound-hard obstacle reconstruction. Proc. 7th Int. Conf. on Mathematical and numerical Aspects of Waves (WAVES'05), J. Comput. Appl. Math., (in press), 2006.

[44] R. Kussmaul. Ein numerisches Verfahren zur Lösung des Neumannschen Aussenraumproblems für die Helmholtzsche Schwingungsgleichung. Computing, 4:246$273,1969$.

[45] P. Lax and L. Philips. Scattering Theory. Academic Press, London, 1967.

[46] R. Leis. Initial Boundary Value Problems in Mathematical Physics. John Wiley, New York, 1986.

[47] L. Levine. A uniqueness theorem for the reduced wave equation. Comm. Pure Appl. Math., 17:147-176, 1964.

[48] C. Liu. Inverse obstacle problem: local uniqueness for rougher obstacles ans the identification of a ball. Inverse Problems, 13:1063-1069, 1997.

[49] E. Martensen. Über eine Methode zum räumlichen Neumannschen Problem mit einer Anwendung für torursartige Berandungen. Acta Math., 109:75-135, 1963.

[50] A. Maue. Über die Formulierung eines allgemeinen Beugungsproblems durch eine Integralgleichung. Zeit. Physik, 126:601-618, 1949.

[51] W. McLean. Strongly Elliptic Systems and Boundary Integral Equations. Cambridge University Press, 2000.

[52] R. Potthast. A fast new method to solve inverse scattering problems. Inverse Problems, 12:731-742, 1996.

[53] R. Potthast. Fréchet differentiability of the solution to the acoustic neumann scattering problem with respect to the domain. J. Inverse Ill-posed Prob., 4:67-84, 1996.

[54] R. Potthast. A point source method for inverse acoustic and electromagnetic obstacle scattering problems. IMA J. Appl. Math., 61, no.2:119-140, 1998.

[55] R. Potthast. On the convergence of a new newton-type method in inverse scattering. Inverse Problems, 17:1419-1434, 2001. 
[56] R. Potthast. Sampling and probe methods - an algorithmical review. Computing, 75:215-235, 2005.

[57] R. Potthast and J. Schulz. A multiwave range test for obstacle reconstructions with unknown physical properties. (to appear).

[58] A. Roger. Newton kantorovich algorithm applied to an electromagnetic inverse problem. IEEE Trans. Ant. Prop., AP-29:232-238, 1981.

[59] P. Serranho. A hybrid method for inverse scattering for shape and impedance. Inverse Problems, 22:663-680, 2006.

[60] P. Serranho. A hybrid method for inverse scattering for sound-soft obstacles in 3D. subm. to Inverse Problems and Imaging, 2007.

[61] I. Sloan and R. Womersley. Constructive approximation on a sphere. J. Approx. Theory, 103:91-118, 2000.

[62] P. Smith, S. Vinogradov, and E. Vinogradova. Canonical Problems in Scattering and Potential Theory. Chapman \& Hall/CRC, 2001.

[63] A. Sommerfeld. Partial Differential Equations in Physics. Academic Press, New York, 1949 (fifth printing, 1967).

[64] J. Spanier and K. B. Oldham. An Atlas of Functions. Washington, DC: Hemisphere, 1987.

[65] L. Wienert. Die numerische Approximation von Randintegraloperatoren für die Helmholtzgleichung in $\mathbb{R}^{3}$. Dissertation, Göttingen, 1990. 



\title{
Lebenslauf - Curriculum Vitae
}

Name: $\quad$ Pedro Miguel Picado de Carvalho Serranho

Geburtstag: $\quad$ 6. Juni 1979

Geburtsort: $\quad$ Lissabon, Portugal

Staatsangehörigkeit: portugiesisch

\begin{abstract}
Akademische Ausbildung
07/1997 "Abitur" an der Escola Secundária Herculano de Carvalho, Lissabon, Portugal

09/2002 Diplom an der Technischen Universität Lissabon

01/2004 Master an der Technischen Universität Lissabon

01/2007 Einreichung der Dissertation
\end{abstract}

